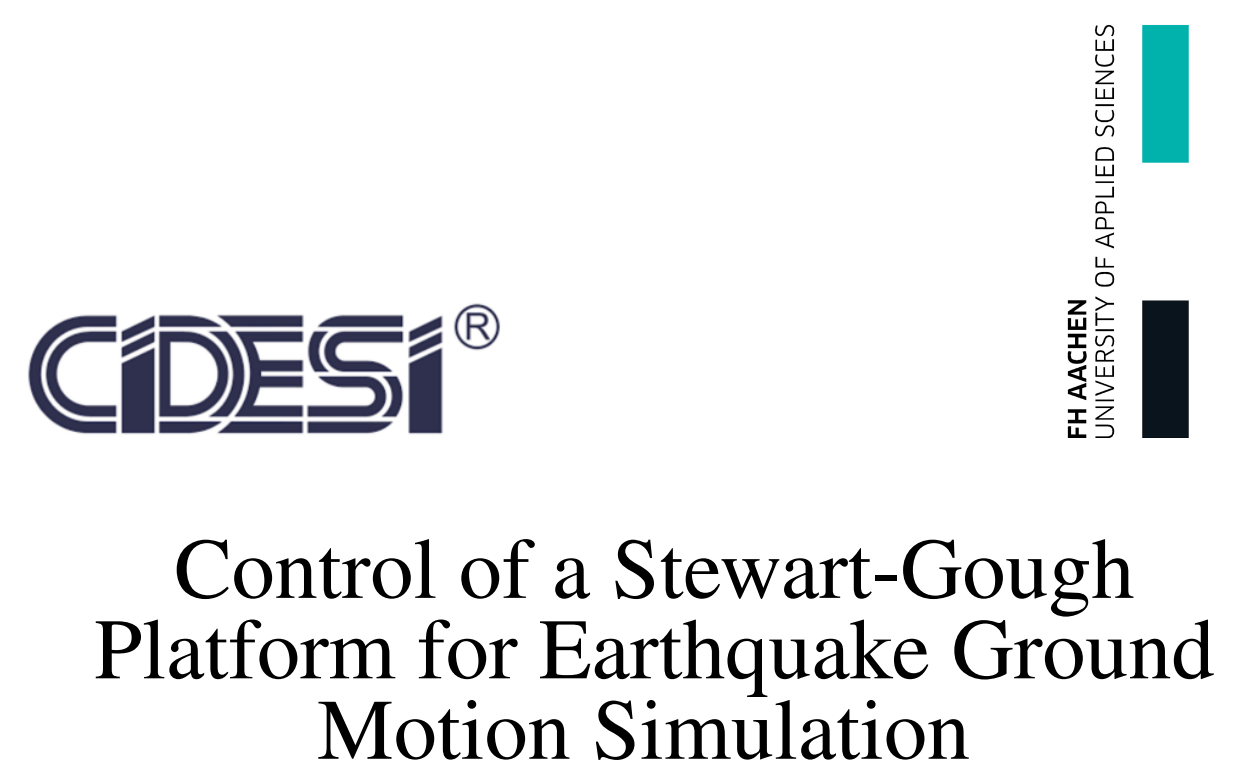

Thesis

Submitted to the Faculty of Fachhochschule Aachen and Centro de Ingeniería y Desarrollo Industrial

BY

Eng. Erick-Franco Alvarado-Requena

PRIMARY ADVISOR:

Ph.D. José Antonio Estrada-Torres

In Partial Fulfillment of the requirements for the degree of Master of Science in Mechatronics 



\section{Declaration of Autorship}

I, Erick Franco Alvarado-Requena, declare that this thesis, "Control of a Stewart-Gough Platform for Earthquake Ground Motion Simulation", is the result of my own work. Any part of this dissertation has not been previously submitted, in part or whole, to any university or institution for a degree or other qualification.

I confirm that all consulted work for others is attributed and the source is always given. Where the work is done with the help of others, these sources of help have been acknowledged.

Signed:

Date: 



\section{Acknowledgments}

First, I would like to acknowledge (CONACYT) for the provided funds for studying the double degree master's program at CIDESI in junction with the University of Applied Sciences, FH Aachen.

Really special thanks to Ph.D. José Antonio Estrada Torres and Dr. Eng. M. Sc. Gengis K. Toledo Ramírez for being my first and second advisor in México, let me work on this project and all the help and support that they provided me in the development of this thesis.

A special thanks to Prof. Dr. rer. nat Klaus-Peter Kämper and Dr. Eng. Jörg Wollert for their support, help and contribution to my formation during my studies at the FH Aachen in Germany. Thank you to my classmates that shared with me a lot of experiences, good times and helped me whenever i needed it, becoming my friends.

Finally, thank you to my mother, grandmother and my grandfather. They were supporting me all the time despite the distance and circumstances, pushing me to reach my goals; without them this would be impossible. 



\begin{abstract}
The Stewart-Gough platform is a six DOF parallel robot manipulator with higher precision, mechanical stiffness and load capacity if compared with conventional serial link arm. In this master thesis, a CAD model of CIDESI Stewart Platform HxCf1 is imported to Simscape as a dynamic model and an application of an inverse dynamics control scheme is proposed; full kinematic equations of the robot are presented. A ground motion movement "profile" consisting on position $(\mathrm{x}, \mathrm{y}, \mathrm{z})$ and orientation $(\alpha, \beta, \gamma)$ based on real earthquake records is loaded and simulated; simulation results illustrate the performance of the HxCf1 robot using the control algorithm.
\end{abstract}





\section{Kurzfassung}

Die Stewart-Gough Plattform ist ein System mit sechs Freiheitsgraden hoher Präzision, mechanischer Steifheit und Tragfähigkeit im Vergleich mit konventionellen Roboterarm Konfigurationen. Diese Master-Abschulssarbeit behandelt ein CAD Modell der CIDESI Stewart HxCf1 Platt-

form, welches nach Simscape importiert als dynamische modell wurde, sowie die Anwendung eines Kontrollschemata inverser Dynamik vorschlägt; es werden die Gleichungen der Kinematik dieser Plattform dargeboten. Die Bodenbewegungen realer Erbbebenaufzeichnungen wurden als Profile der Positionen in $\mathrm{x}, \mathrm{y}, \mathrm{z}$ sowie der Orientierungen alpha, beta, gamma simuliert. Die Simulationsresultate illustrieren die Performance der vorgestellten Steuerungsalgorithmen der HxCf1 Plattform. 



\section{Resumen}

La plataforma Stewart-Gough es un robot paralelo con seis grados de libertad que tiene mayor precisión, rigidez mecánica y capacidad de carga si se compara con un robot serial convencional. En este trabajo de tesis de maestría, un modelo CAD de la plataforma Stewart-Gough HxCf1 desarrollada en CIDESI, es importado a Simscape como modelo dinámico y el uso de un esquema de control de dinámica inversa es propuesto; las ecuaciones cinemáticas completas del robot son presentadas. Un "perfil" de movimiento de la tierra que consiste en posición (x, y, z) y orientación $\alpha, \beta, \gamma)$ basado en registros de terremotos reales es usado como entrada y simulado; los resultados de la simulación ilustran el desempeño del robot HxCf1 usando el algoritmo de control. 



\section{Contents}

Declaration of Autorship . . . . . . . . . . . . . . . iii

Akcnowledgments ...................... . . . V

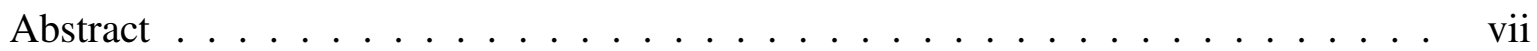

Kurzfassung ......................... . . . . . . . . .

Resumen .......................... . . . . . . . . . .

Contents .......................... . . . . . . .

1 Introduction $\quad 1$

1.1 Motivation ....................... 4

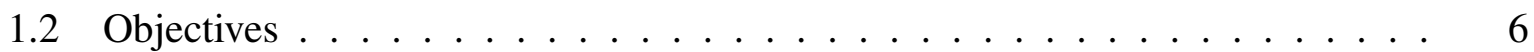

1.2.1 General objective . . . . . . . . . . . . . . . 6

1.2.2 Specific objectives .................... 6

1.3 Justification . . . . . . . . . . . . . . . . . . . . 7

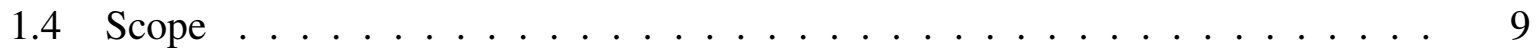

1.5 Hypothesis . . . . . . . . . . . . . . . . . . . 10

1.6 Methodology ............................ 10

2 Theoretical Background $\quad 11$

2.1 Stewart-Gough platform . . . . . . . . . . . . . . . . 11

2.1.1 Stewart-Gough platform configurations . . . . . . . . . . . 13

2.1.2 Stewart-Gough platforms applications . . . . . . . . . . . . 14

2.2 Stewart-Gough Platform kinematics . . . . . . . . . . . . . . 16

2.2 .1 Inverse kinematics . . . . . . . . . . . . . . . . 16

2.3 Control scheme proposal . . . . . . . . . . . . . . . . . . . 19

2.4 Ground motion database . . . . . . . . . . . . . . . . . 20

2.4.1 Earthquake ground motion profile . . . . . . . . . . . . 21

3 CIDESI Hexapod HxCf1 Project 23 


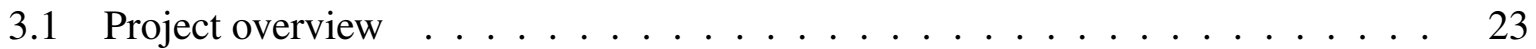

3.2 Previous work on the CIDESI Hexapod HxCf1 project . . . . . . . . . . 24

3.2.1 Industrial Robot project proposal . . . . . . . . . . . . . . . . . 24

3.2.2 Demonstration development for a Hexapod robot . . . . . . . . . 24

3.2.3 Hexapod system for truck motion simulation . . . . . . . . . 25

3.2.4 Alpha prototype of an Hexapod robot . . . . . . . . . . . . 25

3.2.5 Preliminary Design of a Stewart-Gough platform . . . . . . . . 25

3.2.6 Detailed Design of a Stewart-Gough platform . . . . . . . . . 25

3.2 .7 Robot assembly . . . . . . . . . . . . . . . . . . . 26

3.2 .8 Robot Controller . . . . . . . . . . . . . . . . . . 26

3.2.8.1 Hardware . . . . . . . . . . . . . . . . 27

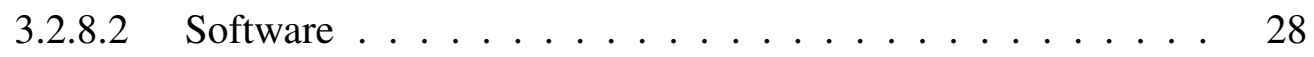

4 CIDESI Hexapod HxCf1 characterization 29

4.1 Inverse Kinematics . . . . . . . . . . . . . . . . . . . . . . . . . . . . . . 29

4.1.1 Base and Mobile platforms configuration . . . . . . . . . 30

4.1.2 Mobile platform position and orientation $\ldots \ldots \ldots . \ldots 34$

4.2 Jacobian . . . . . . . . . . . . . . . . . . . . . . . . . 35

4.3 Frequency work range $\ldots \ldots \ldots \ldots$. . . . . . . . . . . 37

5 Dynamic Model 41

5.1 Simscape dynamic model . . . . . . . . . . . . . . . . . . . . . 41

5.1.1 CIDESI HxCf1 Hexapod Simscape model . . . . . . . . . . . . . 42

5.1 .2 Positions and Orientations $\ldots \ldots \ldots \ldots \ldots$

5.1 .3 Velocities . . . . . . . . . . . . . . . . . . . 44

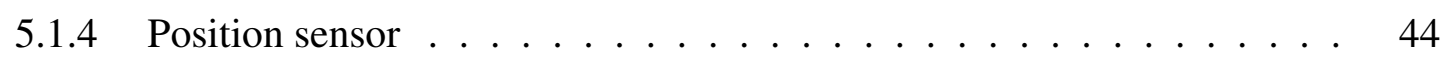

5.1.5 Earthquake ground motion profile . . . . . . . . . . 44

5.2 Linear actuator parameters $\ldots \ldots \ldots$. . . . . . . . . . . . . 47

5.2 .1 Electrical system of equations $\ldots \ldots \ldots \ldots$. . . . . . . . 47

5.2.2 Mechanical system of equations . . . . . . . . . . . . . 49

5.2 .3 DC Motor model . . . . . . . . . . . . . . . . . . . . 50

6 PID Controller $\quad 53$

6.1 Pololu Jrk default motor controller . . . . . . . . . . . . . . . . . . 54

6.2 Ziegler Nichols tuning $\ldots \ldots \ldots \ldots$

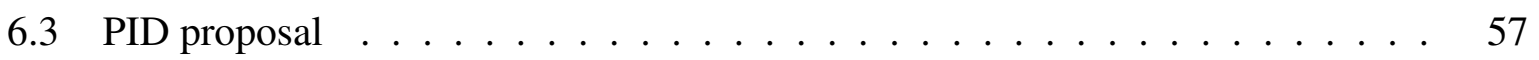


7 Earthquake simulations $\quad 59$

7.1 Default PD simulation . . . . . . . . . . . . . . . . 59

7.2 Proposed PID simulation . . . . . . . . . . . . . . . . . . 64

8 Experimentation $\quad 69$

8.1 Default PD experimental results . . . . . . . . . . . . . . . 69

8.2 Proposed PD experimental results . . . . . . . . . . . . . . 72

9 Conclusions and Further Work $\quad 75$

$\begin{array}{ll}\text { Bibliography } & 77\end{array}$

$\begin{array}{ll}\text { Acronyms } & 81\end{array}$

$\begin{array}{lr}\text { List of Figures } & 85\end{array}$

$\begin{array}{ll}\text { List of tables } & 87\end{array}$

$\begin{array}{lr}\text { A Chronogram } & 89\end{array}$

$\begin{array}{ll}\text { B Positions and Orientations } & 91\end{array}$

$\begin{array}{ll}\text { C Velocities } & 95\end{array}$

$\begin{array}{ll}\text { D Pololu LACT6P linear actuator datasheet } & 101\end{array}$ 



\section{Introduction}

Robotics is a relatively modern field of study and technological research that includes several fields of engineering like mechanical, electronic, information, computer science, and others; robotics deals with the design, construction, operation, and use of robots, as well as computer systems for their control, sensory feedback, and information processing. Since its inception, robotics research has changed in numerous and innovative ways since the beginning of the eighties, when parallel robots were promoted as the last solution for automated manufacturing [18].

In the last decades, there has been a big increment of activity in robotics in research and in terms of generating endless possibilities of applications by the general users. This period brought also a technological maturation of robots, from the simple applications like pick and place, painting and welding robots, to sophisticated assembly robots for very specific applications like inserting integrated circuit chips onto printed circuit boards. Several areas of robotic automation have now become standard on the factory floor and the field is on the verge of a new explosion to areas of growth involving hazardous environments, minimally invasive surgery, and micro electro-mechanical mechanisms [32].

The most common types of used industrial robotic systems, as seen in Figure 1-1 are serial manipulators which are several linkages serially connected like a chain to give a desired motion to the end-effector such as robot arms and parallel manipulators where several serial chains are used to control a single platform such as delta robots [20]. 


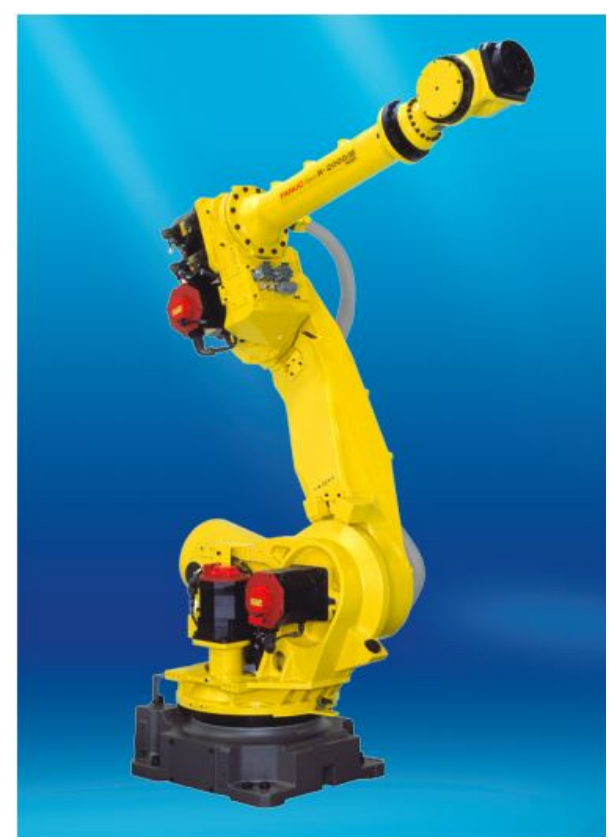

(a) Fanuc R-2000iB serial robot.

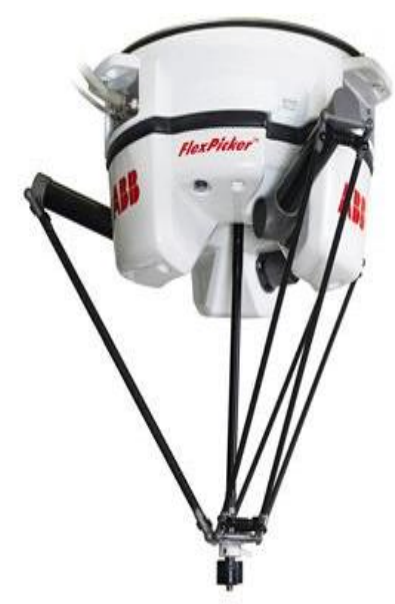

(b) Delta robot ABB Flexpicker.

Figure 1-1: Common used industrial robotic systems. Taken from [6] [17].

Parallel manipulators offer some advantages such as precision, mechanical stiffness and higher load capacity, due to its parallel structure if compared with serial manipulators; on the other side, a disadvantage of parallel manipulators is the workspace limitations due to the link lengths and the mechanical limits of the passive joints [30]. The most representative parallel manipulator is the Stewart-Gough platform. It consists of a fixed base and a mobile platform, connected by six prismatic actuators or legs; this configuration provides six Degrees Of Freedom (DOF ), three translational and three rotational, on its mobile platform.

Parallel manipulators can be used to work as devices called shaking tables in order to develop different experimental techniques to test the response of structures like houses or buildings to verify their seismic performance. This devices can shake scaled slopes, structural models or building components with a wide range of simulated ground motions, including reproductions of recorded earthquakes time-histories.

Modern shaking tables consist of a rectangular platform that is driven in up to six DOF by servo-hydraulic or other types of actuators with the test specimens fixed to the platform (see Figure 1-2); these are shaken following representative conditions of true earthquake ground motions, often to the point of failure [14]. 


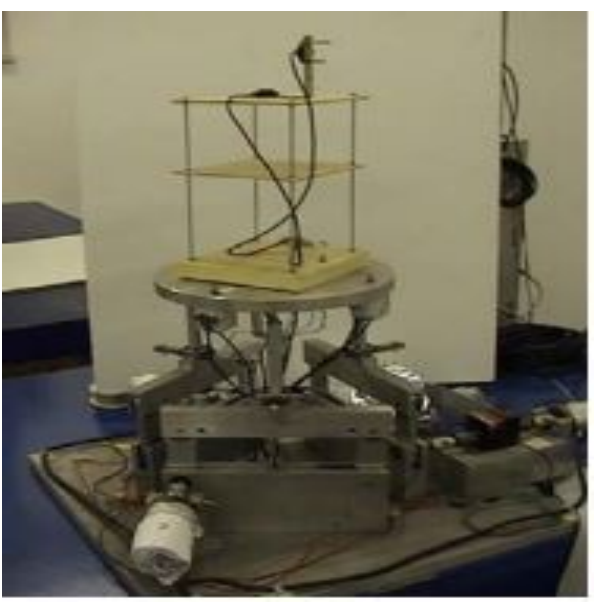

(a) Shaking table for scale buildings

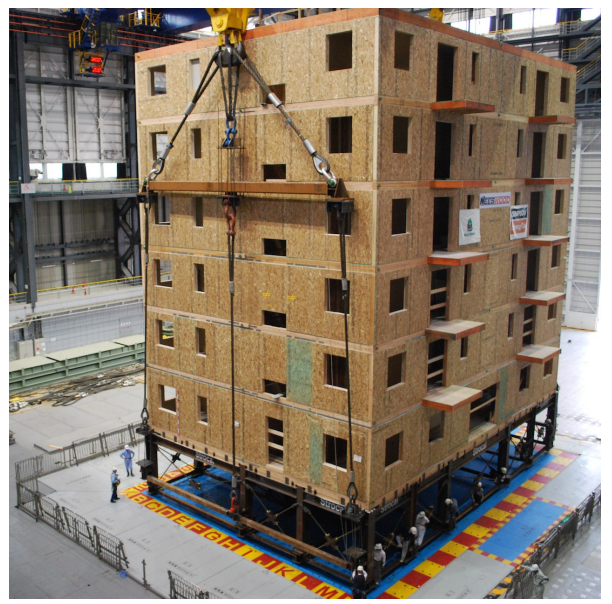

(b) Largest shake table in the world

Figure 1-2: Examples of shaking tables. Taken from [14] [8].

The Centro de Ingeniería y Desarrollo Industrial (CIDESI) Hexapod robotic system is a project that aims to develop an industrial grade robotic system that provides functionalities similar to those usually found in an industrial robot; the CIDESI Hexapod has been designed to be operated by a human operator (User), or by another system or device (Application) like, e.g. a vision system.

Generally, a Hexapod robot system consists of a Robot (mechanical structure), controller, Teach Pendant, controller/computer, user documentation and accessories. These are the basic components that are generally included in any serious commercial robotic system. The "Hexapod CIDESI project" is divided at least in three phases, with the development of one prototype with increased capabilities in each phase; the current prototype (see Figure 1-3) corresponds to the phase I, which is called "Hexapod CIDESI phase 1", better known by its nickname: "HxCf1" [29, 36]. In the Phase I, the robot is mainly intended as a low cost industrial-like experimental prototype [21].

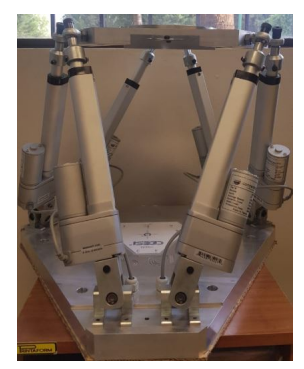

Figure 1-3: HxCf1 Hexapod CIDESI project. Taken from [36]. 


\subsection{Motivation}

An earthquake is a sudden shaking movement of the surface of the earth that cannot be predicted or controlled; it can also be known as a quake, tremblor or tremor. The range of an earthquake in size can vary from very weak ones that cannot be felt to violent enough to toss people around and destroy whole cities.

The seismicity or seismic activity of an area refers to the frequency, type and size of earthquakes experienced over a period of time. Earthquakes typically occur along tectonic plate boundaries, but some occur along fault lines in the middle of the plates as well. Countries around the Pacific Rim are heavily affected, as are many countries in the Mediterranean region and western and southern Asia(see Figure 1-4) [16].

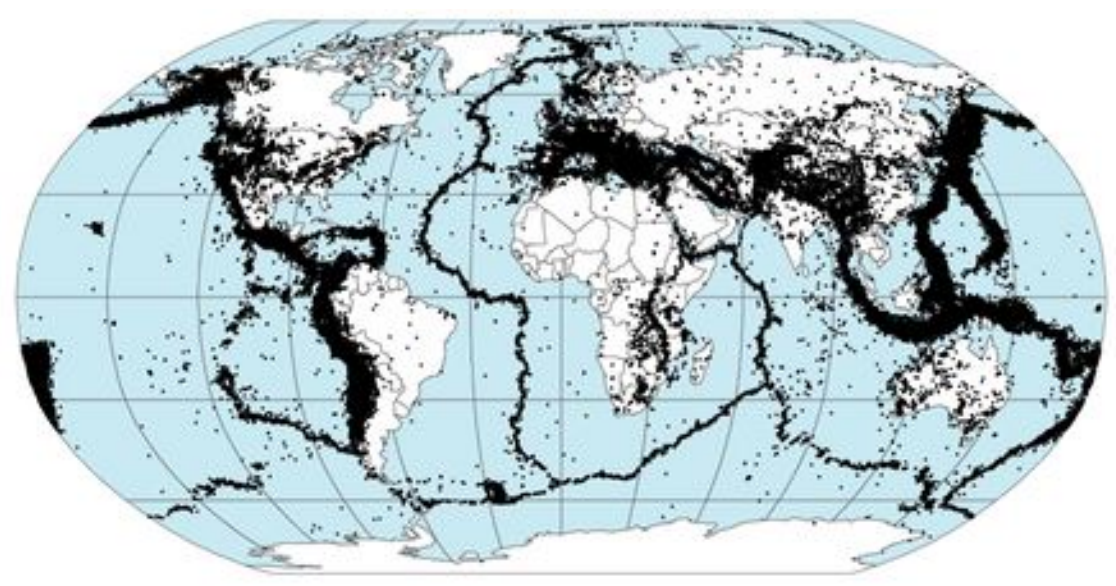

Figure 1-4: Occurrence of Earthquakes Globally (Digital Tectonic Activity Map (DTAM ), 2002). Taken from [4].

Earthquakes represent a major hazard for populations around the world and according to statistics, its the first cause of death toll due to natural disasters (see Figure 1-5). Ground shaking is the most familiar effect of earthquakes and because of this buildings can be damaged or destroyed, people and animals have trouble standing up or moving around and objects can be tossed around.

Taking into account the effects of an earthquake, its very important to remark that while many people are killed in earthquakes, none of them are actually killed directly by the shaking; human losses are caused by the construction of buildings that are prone to failure in earthquakes (see Figure 1-6). 


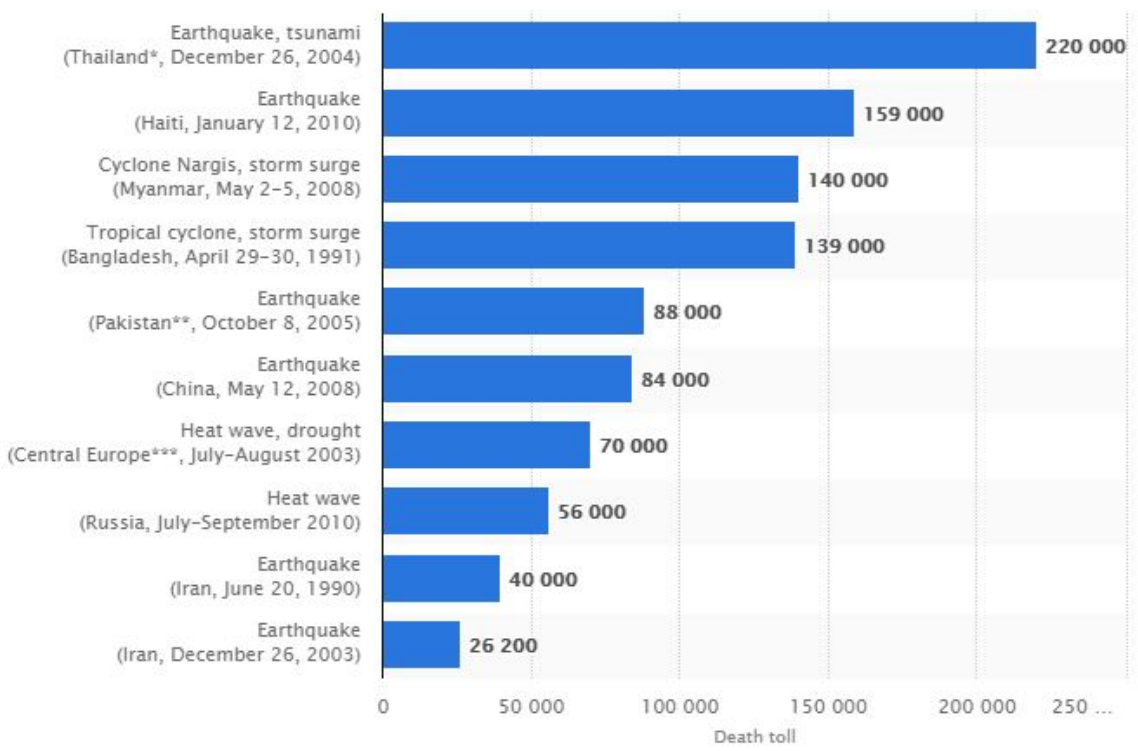

Figure 1-5: The 10 most significant natural disasters worldwide by death toll from 1980 to 2018. Taken from [1].

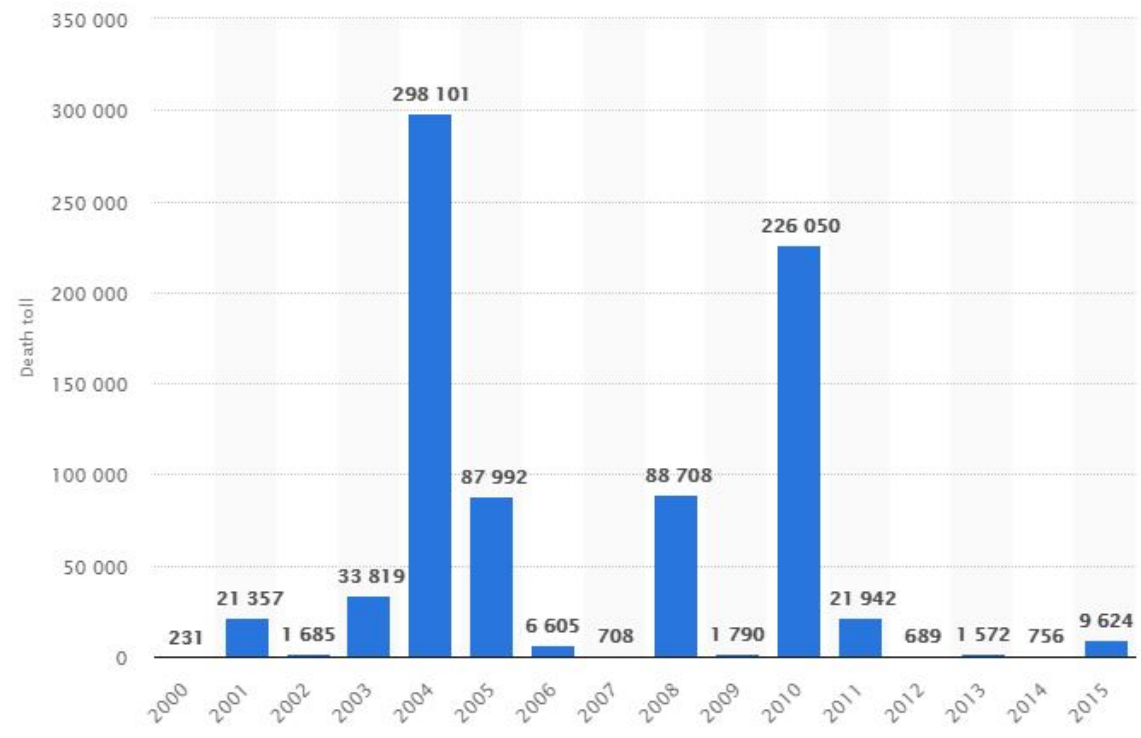

Figure 1-6: Global death toll due to earthquakes from 2000 to 2015. Taken from [7].

The use of shaking tables is a powerful method to test the seismic capacity of a building using scale models under controlled conditions. The scale models tests represent a economical option compared with full-scale tests and also are practical because it is possible to observe the damages that can be generated to the building [26]. 
Using the Berkeley University records from past earthquakes in addition with a scale structure, a shaking table can be reproduced and tests can be performed in order to obtain important data to prevent building failures and human losses when a real quake happen.

\subsection{Objectives}

This section presents the general and specific objectives of the master thesis project.

\subsubsection{General objective}

Reproduce ground shaking movements of different frequencies corresponding to real earthquakes based on Pacific Earthquake Engineering Research Center recorded data using HxCf1 CIDESI Hexapod of 6 DOF in the period of August-November 2019, enhancing the performance obtained using the default controller.

\subsubsection{Specific objectives}

Several specific objectives are defined, in order to provide milestones that ultimately lead to the fulfillment of the general objective.

1. Thesis protocol document

2. Research of the state of the art

3. HxCf1 platform characterization

4. Generate a model proposal

5. Test earthquake profiles

6. Validate system model

7. Master Thesis

8. Thesis dissertation 


\subsection{Justification}

According to the International Federation of Robotics (IFR ) statistics of 2018, in 2017 robot sales increased by 30\%, representing a new peak for the fifth year in a row. Since 2010, the demand for industrial robots has accelerated considerably due to the ongoing trend toward automation and continued innovative technical improvements in industrial robots. In the last five years, between 2012 and 2017, the average robot sales increase was 19\% per year. In the period of 2015-2017 the average annual increase was about 310,000 units. This is a clear indication of the tremendous, accelerating rise in demand for industrial robots worldwide and the importance of research and development of new ones. Figure 1-7 [5].

A prediction for the annual worldwide supply of robots and the stock of operation robots based on the last years statistics shows that, year by year, robotic applications are growing and it is going to be more and more profitable and important in the industry daily life (Figure 1-8).

Taking into account the importance of robotics improvement and adding a very important human necessity as try to protect people from natural disasters, the development of an earthquake reproducing parallel robot can help to test and enhance building structures and devices which function is to decrease or nullify damages generated by ground shaking movements. As shown in Figure 1-9, every year there are more than 1,300 earthquakes with Richter scale of 5.0 or more in the whole world.

In addition, CIDESI is working in the development of a wireless sensor network for the observation and monitoring of the structural health; both projects can work together in order to bring simulations to real life tests and results. 


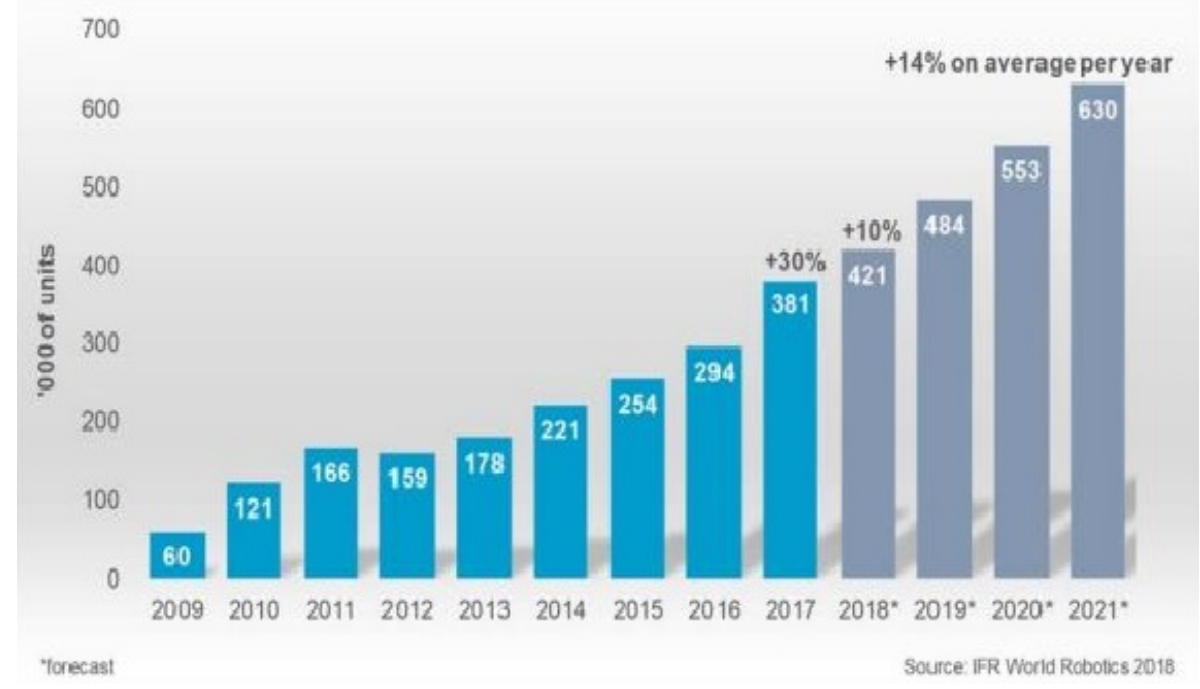

Figure 1-7: Estimated annual worldwide supply of industrial robots 2009-2017 and 2018*$2021 *$. Taken from [5]

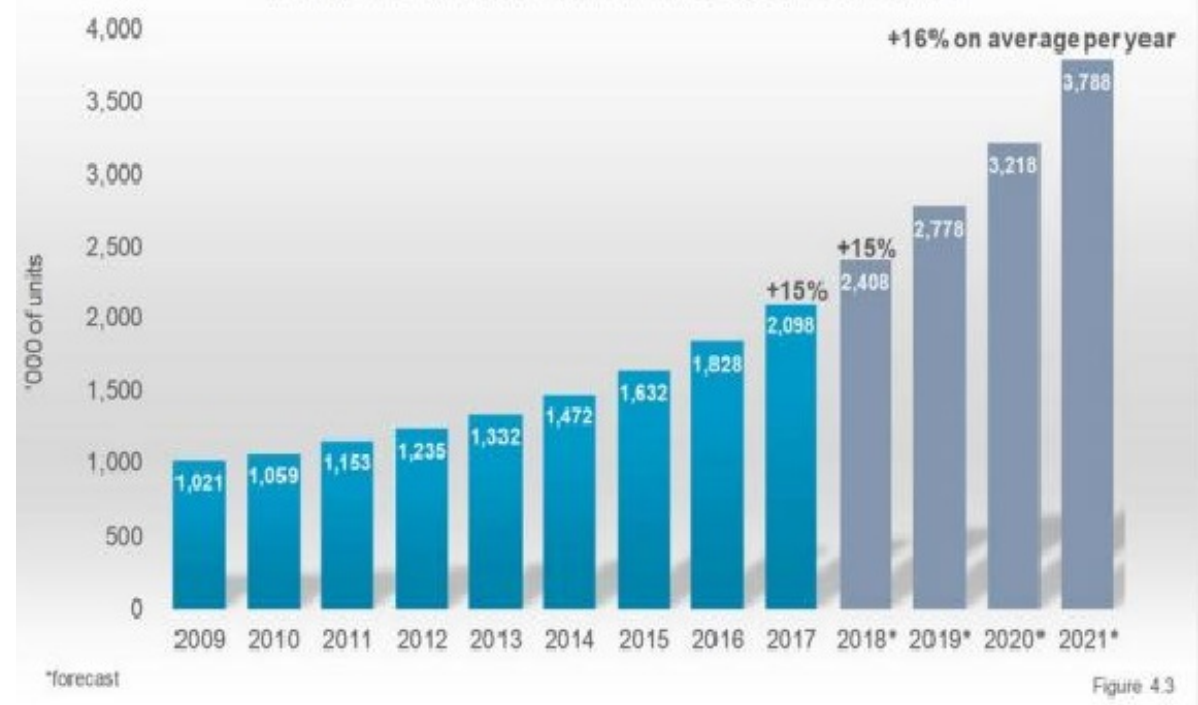

Figure 1-8: Estimated annual worldwide operational stock of industrial robots 2009-2017 and $2018 *-2021 *$. Taken from [5] 


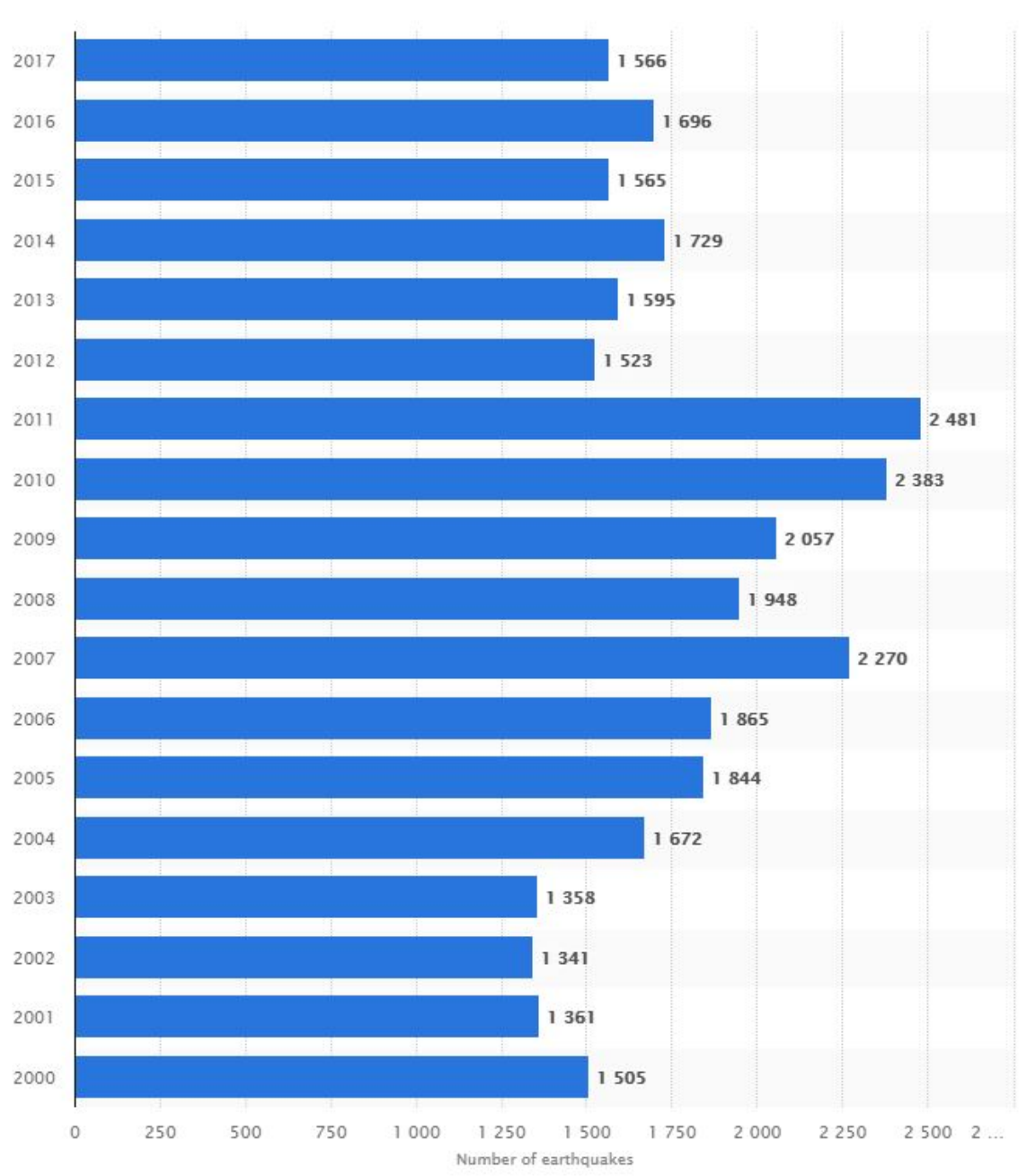

Figure 1-9: Development of the number of earthquakes (M5+) worldwide from 2000 to 2017. Taken from [3]

\subsection{Scope}

In this master thesis, previous work on static analysis will be complemented with dynamic analysis of the Hexapod HxCf1 and using the command line interface profile loader, ground shaking movements of different frequencies will be reproduced. 


\subsection{Hypothesis}

Using an adequate proposed control scheme, it is possible to reproduce ground shaking movements of different frequencies corresponding to real earthquakes based on Pacific Earthquake Engineering Research Center recorded data using HxCf1 CIDESI Hexapod of 6 DOF.

\subsection{Methodology}

The steps to follow in order to develop the master thesis project "Control of a Stewart-Gough platform for earthquake motion Simulation" are listed below.

1. Theoretical background research.

2. HxCfl hexapod characterization.

3. DC motor electrical and mechanical values obtaining.

4. DC motor Matlab model proposal.

5. Develop dynamic model equations.

6. Obtain specific parameters for the proposed model.

7. Generate earthquake profiles for a determined frequency in the working range.

8. Simulation of the Hexapod using Matlab.

9. Experimentation.

10. Obtain and compare results of simulation and experimental tests.

11. Define conclusions and further work.

12. Project documentation as a Master Thesis. 


\section{Theoretical Background}

This chapter presents an introduction to the topics of Parallel robots, the Stewart-Gough Platform (SP ) as well as its applications and kinematics, followed by a literature review of scientific publications and related works.

\subsection{Stewart-Gough platform}

The Hexapod Stewart - Gough, whose design was proposed by VE Gough (1956-1957), and studied and presented by D. Stewart in 1965, is a robot designed in a closed chain, in which two platforms are connected; a platform, considered as the base of the robot, and a mobile platform in parallel connection, considered the upper part of it. Both joined by six actuators or linear extensible arms, where their points of union with the platforms are mechanical joints with more than two degrees of movement, which has 12 possible polynomial forms of movement. The design provides high performance in precision and strength compared to serial manipulators [35].

Hexapod positioners are often referred to as Stewart-Gough Platforms. A hexapod is based on a 6-axis (XYZ, Pitch, Roll, Yaw) actuator system arranged in parallel between a top and bottom platform. Devices placed on the top plate can be moved in the six DOF in which it is possible for a freely-suspended body to move. These are the three linear movements $\mathrm{x}, \mathrm{y}, \mathrm{z}$ (lateral, longitudinal and vertical), and the three rotations pitch, roll and yaw (see Figure 2-1). 


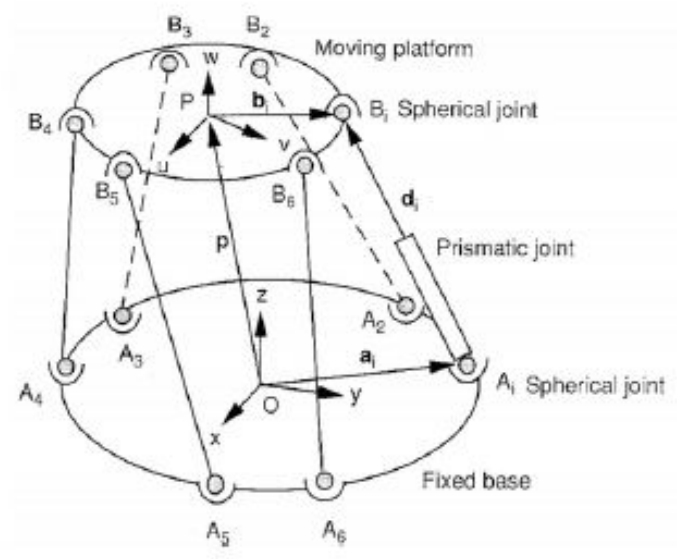

Figure 2-1: Stewart-Gough platform. Taken from [37].

Parallel kinematics precision positioning systems have many advantages over serial kinematics stages such as lower inertia, improved dynamics, smaller package size and higher stiffness; in addition Hexapods are more flexible than conventional 6 axis positioners [31]. However, a major drawback is that the workspace of parallel robots is severely restricted compared to equivalent serial robots.

Since a parallel structure is a closed kinematics chain, all legs are connected from the origin of the tool point in the base to the mobile base by a parallel connection. This connection allows a higher precision and a higher velocity [31].

Complex kinematics and dynamics often lead to model simplifications decreasing the accuracy and because of this, accurate kinematic and dynamic identification is needed in order to overcome this problem. The kinematic and dynamic modelling of SP is extremely complicated in comparison with serial robots.

For a parallel manipulator, inverse kinematics calculations are can be done straight forward which represents no complexity deriving the equations. However, forward kinematics calculations of SP are very complicated and difficult to solve since it requires the solution of many non-linear equations. Moreover, the forward kinematic problem generally has more than one solution [39].

The dynamic modelling of parallel manipulators is quite complicated because of their closedloop structure, coupled relationship between system parameters, high non-linearity in system dynamics and kinematic constraints. Robot dynamic modelling can be also divided into two topics: inverse and forward dynamic model. The inverse dynamic model is important for system control while the forward model is used for system simulation. The dynamic analysis of parallel 
manipulators has been traditionally performed through several different methods such as the Newton-Euler method, the Lagrange formulation, the principle of virtual work and the screw theory [39].

\subsubsection{Stewart-Gough platform configurations}

There are different geometrical configurations for this parallel robot, each one of them has singular characteristics in the difficulty of the implementation of the control system, manufacturing, performance in the kinematics and the workspace (see Figure 2-2). The 3 most common configurations are [31]:

- Minimal Symmetric Simplified Manipulator (MSSM ). This configuration that is the simplest of all consists of a fixed base, a mobile base, 6 actuators and 6 connectors or unions, 3 of these in the mobile base and the other 3 in the fixed base with a triangular symmetrical separation, simplifying the number of connectors facilitates the analysis of the direct kinematics of the mechanism, and with this the control of the same, however each connector is linked to two actuators and should have three degrees of freedom so that the The design of these joints is complicated, has many physical restrictions and is very difficult to manufacture.

- Triangular Symmetric Simplified Manipulator (TSSM ). This configuration has 6 joints in the fixed base and 3 in the mobile base, which is why it presents the same problem of concentric joints.

- Symmetric simplified Manipulator (SSM ). This configuration avoids the problems of concentric joints using a union for each actuator, however the number of singular positions is increased, the analysis of direct kinematics becomes complicated and it is necessary to use long computational methods reaching up to 40 with singular configuration [24]. 


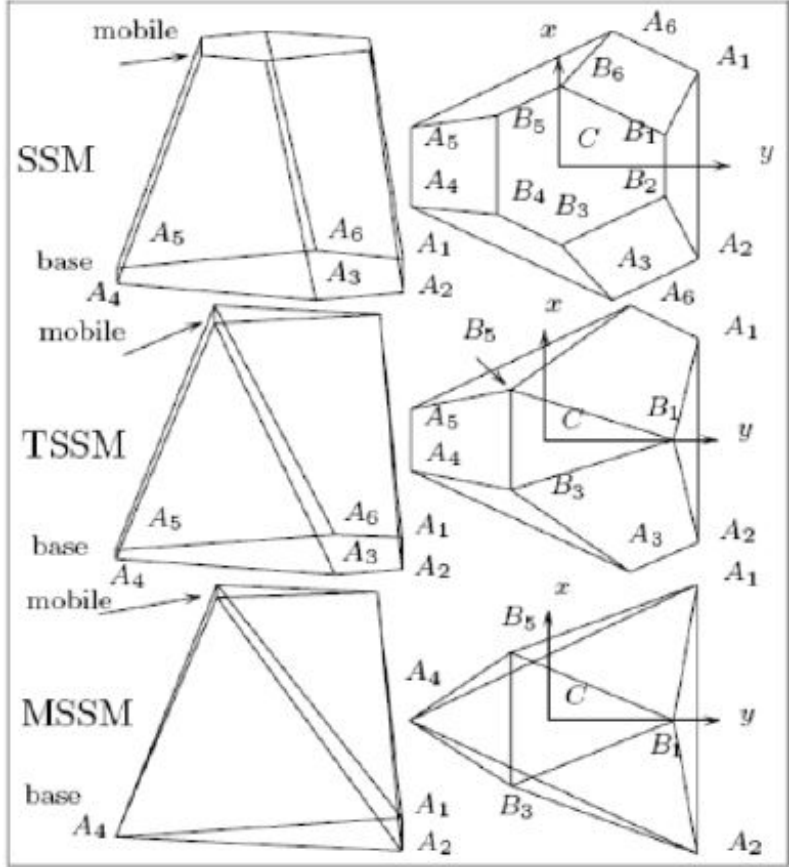

Figure 2-2: Geometrical configurations for Stewart-Gough platforms of 6 DOF. Taken from [31].

\subsubsection{Stewart-Gough platforms applications}

Stewart Gough platform was proposed originally as a mechanism for flight simulation but with the time, a lot of new applications were proposed and developed; a list of applications is listed below.

- Wave compensation. Ship mounted cranes are intensively used in the shipping industry to load and unload ships. As loading and unloading of cargo can take place at sea, the crane and cargo is exited by the motion of the ship. The excitation can cause the hoisted cargo to pendulate uncontrollably and coerce the crane operator to shut down the operation for safety reasons. Crane operations at sea are thus dependent on no or only small wave-exited motion of the ship to avoid uncontrollable pendulation of the hoisted cargo. To increase the available work time of crane ships, a design of a hydraulic Stewart platform can be used to work as a stable foundation for the ship crane and suppress the motion from the waves as shown in Figure 2-3 [28]. 


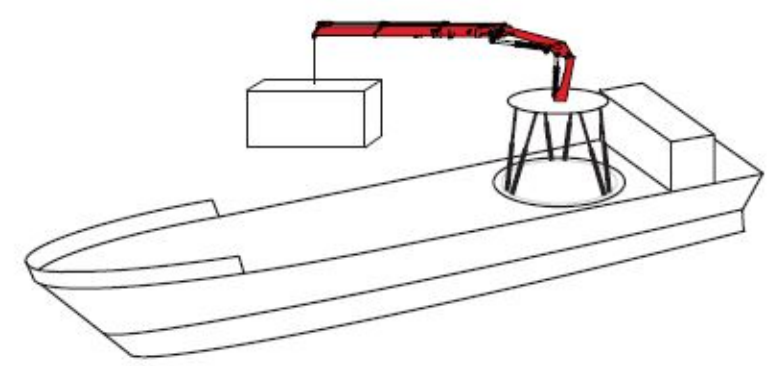

Figure 2-3: Ship Crane mounted on Stewart platform. Taken from [28].

- Spherical radio telescopes. For the requirement of trajectory tracking of large spherical radio telescopes, a large fine tuning platform based on the Stewart platform design can be used to assure the performance. Design of the SP is showed in Figure 2-4.

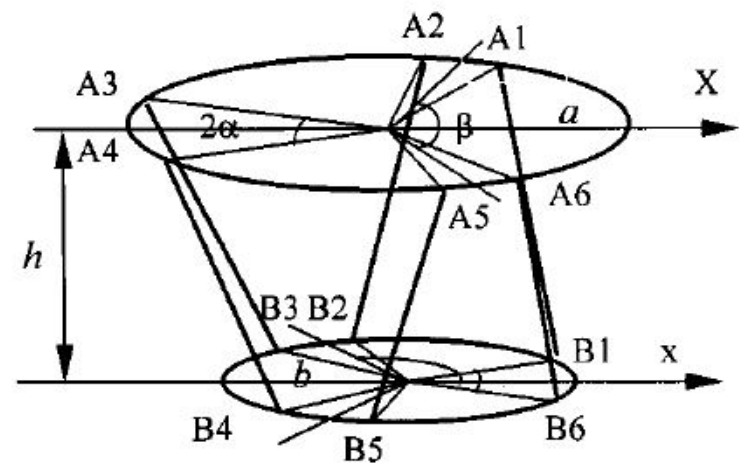

Figure 2-4: Proposed design structure of the fine tuning platform. Taken from [19].

- Surgical robotics. To simulate body organ motion due to breathing, heart beats, or peristaltic movements, a miniaturized Hexapod (Figure 2-5) can be used to translate and rotate phantom tissue [38]. This can be very useful in surgical robotics and with high importance looking forward in medicine innovations.

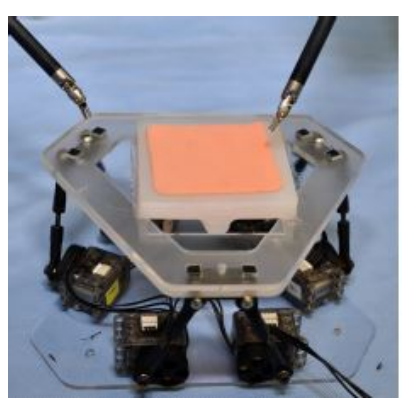

Figure 2-5: Miniaturized Stewart platform to simulate anatomical movements. Taken from [38]. 


\subsection{Stewart-Gough Platform kinematics}

Kinematics studies the motion of bodies without consideration of the forces or moments that cause the motion. Robot kinematics refers the analytical study of the motion of a robot manipulator. Formulating the suitable kinematics models for a robot mechanism is very crucial for analysing the behaviour of industrial manipulators; kinematics to take into account are inverse and forward.

\subsubsection{Inverse kinematics}

Given the robot's end-effector location, inverse kinematics equations calculate the joint angles required to move the end-effector to that location.

In order to calculate inverse kinematics, the configuration shown in Figure 2-6 is used and the following equations are needed to obtain a solution:
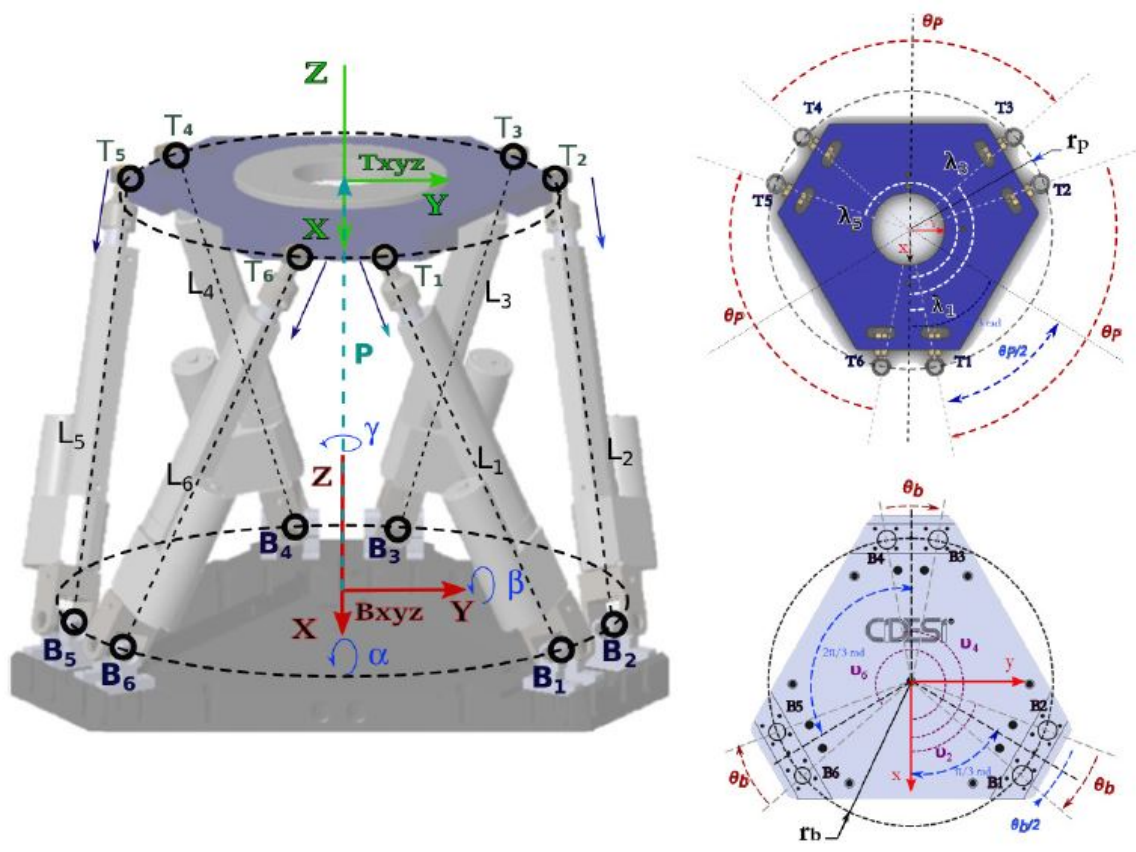

Figure 2-6: Hexapod configuration. Taken from [13] [27].

The following position vectors of the connexion points on its corresponding coordinate system are derived: 


$$
\begin{gathered}
G T_{i}=\left[\begin{array}{c}
r_{p} \cos \left(\lambda_{i}\right) \\
r_{p} \sin \left(\lambda_{i}\right) \\
0
\end{array}\right] \\
B_{i}=\left[\begin{array}{c}
r_{b} \cos \left(v_{i}\right) \\
r_{b} \sin \left(v_{i}\right) \\
0
\end{array}\right]
\end{gathered}
$$

We refer to the origin of the moving platform as $\mathrm{G}$. The position vector of $\mathrm{G}$ w.r.t. the $B_{X Y Z}$ coordinate system is denoted as $P=\left[P_{x} P_{y} P_{z}\right]^{T} \cdot{ }^{B} R_{T}$ is the rotation matrix defined by the roll $(\alpha)$, pitch $(\beta)$, and yaw $(\gamma)$ angles. For any rotation performed on the moving platform, this matrix maps any vector represented in $T_{X Y Z}$ coordinates to its corresponding $B_{X Y Z}$ coordinates. Define $R_{x}(\alpha)$ as the matrix that represents the rotation $\alpha$ about the fixed $\mathrm{x}$-axis, in the same manner $R_{Y}(\beta)$ and $R_{Z}(\gamma)$ represent rotations about the fixed y and z-axes. We have that

$$
\begin{aligned}
R_{X}(\alpha)= & {\left[\begin{array}{ccc}
1 & 0 & 0 \\
0 & \cos \alpha & -\sin \alpha \\
0 & \sin \alpha & \cos \alpha
\end{array}\right] } \\
R_{Y}(\beta)= & {\left[\begin{array}{ccc}
\cos \beta & 0 & \sin \beta \\
0 & 1 & 0 \\
-\sin \beta & 0 & \cos \beta
\end{array}\right] } \\
R_{Z}(\gamma)= & {\left[\begin{array}{ccc}
\cos \gamma & -\sin \gamma & 0 \\
\sin \gamma & \cos \gamma & 0 \\
0 & 0 & 1
\end{array}\right] }
\end{aligned}
$$

There are twelve different combinations for the order of rotation of this angles, considering also combinations of three rotations with respect to only two axes, that can represent arbitary 3D rotations. In this document we adopt the order $\alpha, \beta, \gamma$ all of them w.r.t. the fixed platform, that is:

$$
{ }^{B} R_{T}=R_{Z}(\gamma) R_{Y}(\gamma) R_{X}(\alpha)
$$




$$
=\left[\begin{array}{ccc}
\cos \beta \cos \gamma & \cos \gamma \sin \alpha \sin \beta-\cos \alpha \sin \gamma & \sin \alpha \sin \gamma+\cos \alpha \cos \gamma \sin \beta \\
\cos \beta \cos \gamma & \cos \alpha \cos \gamma+\sin \alpha \sin \beta \sin \gamma & \cos \alpha \sin \beta \sin \gamma-\cos \gamma \sin \alpha \\
-\sin \beta & \cos \beta \sin \alpha & \cos \alpha \cos \beta
\end{array}\right]
$$

Thus, the vector $L_{i}$ corresponding to the link $i$ is obtained as follows (in $B_{X Y Z}$ coordinates).

$$
L_{i}=R_{X Y Z} G T_{i}+P-B_{i}, i=1, \ldots, 6
$$

that is,

$$
L_{i}=\left[\begin{array}{c}
r_{p}[\sin (\alpha) \sin (\beta) \cos (\gamma)-\cos (\alpha) \sin (\gamma)] \sin \left(\lambda_{i}\right)+r_{p} \cos (\beta) \cos (\gamma) \cos \left(\lambda_{i}\right)+x-r_{b} \cos \left(v_{i}\right) \\
r_{p}[\sin (\alpha) \sin (\beta) \sin (\gamma)+\cos (\alpha) \cos (\gamma)] \sin \left(\lambda_{i}\right)+r_{p} \cos (\beta) \sin (\gamma) \cos \left(\lambda_{i}\right)+y-r_{b} \sin \left(v_{i}\right) \\
r_{p} \operatorname{coa}(\alpha) \cos (\beta) \sin \left(\lambda_{i}\right)-r_{p} \sin (\beta) \cos \left(\lambda_{i}\right)+z
\end{array}\right]
$$

and their lengths, $l_{i}$, correspond to the actuator positions

$$
l_{i}=\left\|L_{i}\right\|
$$

After obtaining inverse kinematics, a dynamic model of the Stewart-Gough platform may be derived using the Lagrange formulation [22]. Kinetic and potential energies of the moving platform and the legs, each one consisting of a piston and a cylinder, are obtained in compact expressions. Lagrangian formulations are taken in compact symbolic forms in order to make the expression understandable. The matrix equation is showed below:

$$
M^{\prime}\left(X_{p}\right) \ddot{X}_{p}+V_{m}^{\prime}\left(X_{p}, \dot{X}_{p}\right) \dot{X}_{p}+G^{\prime}\left(X_{p}\right)=J^{T}\left(X_{p} \tau\right)
$$

Where $M^{\prime}($.$) is the mass matrix, V_{m}^{\prime}($.$) is the velocity product term, G^{\prime}($.$) is the gravitational$ force, $\mathrm{J}($.$) is the Jacobian matrix and \tau \in R^{6}$ is the generalized actuator force at each actuator all with respect to the based frame.

Dynamic formulation of the Hexapod in the joint space coordinates can be described by transforming the dynamics written in the task-space coordinates starting with the Jacobian Matrix $J($.$) :$

$$
\dot{x}=J^{-1} \dot{q},
$$


where $\dot{q} \in R^{6}$ is the six legs velocity vector. A dynamic matrix equation in the joint space coordinates will be obtained as follows:

$$
M(q) \ddot{q}+V_{m}(q, \dot{q}) \dot{q}+G(q)=\tau
$$

where

$$
\begin{gathered}
M(q)=J^{-T} M^{\prime}\left(X_{p}\right) J^{-1}, \\
V_{m}(q, \dot{q})=J^{-T} M^{\prime}\left(X_{p}\right) J^{-1} \dot{J J}^{-1}+J^{-T} V_{m}^{\prime}\left(X_{p}, \dot{X}_{p}\right) J^{-1}, \\
G(q)=J^{-T} G^{\prime}\left(X_{p}\right),
\end{gathered}
$$

Where $q \in R^{6}$ represents the six link displacement vector.

\subsection{Control scheme proposal}

In order to linearise and decouple robot manipulator dynamics, inverse dynamic control is used; possible non-linearities such as Coriolis and centrifugal terms as well as gravity terms can be compensated by adding these terms to the control input. However, this type of feedback linearising controller is much more complicated and it's conception is not straightforward for the robot manipulator dynamics [23].

To cancel non-linear terms and decouple the dynamics of each link is used an inverse dynamics control of the form:

$$
u=M(q) u_{0}+C(q, \dot{q}) \dot{q}+G(q)
$$

applied to (2.3). The mass matrix, $M(q)$, is a symmetric positive definite matrix which verifies:

$$
\lambda_{m} I \leq M(q) \leq \lambda_{M} I
$$

where $\lambda_{m}\left(\lambda_{M}<\infty\right)$ denotes the strictly positive minimum(maximum) eigenvalue of $M(q)$ for all configuration $q$. These properties show the regularity of the inertia matrix. The controller of (2.7) and the regularity property of $M(q)$, yield a set of $n$ decoupled linear systems.

$$
\ddot{q}=u_{0}
$$


where $u_{0}$ is an auxiliary control input to be designed. A typical choice for $u_{0}$ is:

$$
u_{0}=\ddot{q}_{d}+K_{D}\left(\dot{q}_{d}-\dot{q}\right)+K_{P}\left(q_{d}-q\right)
$$

which leads to the error equation

$$
\ddot{\tilde{q}}+K_{D} \dot{\tilde{q}}+K_{P} \tilde{q}=0
$$

where $\tilde{q}=-e$.

The error equation, (2.11), is exponentially stable by a suitable choice of the matrices $K_{D}$ and $K_{P}$; equation (2.11) can be rewrite in its state space form, $\dot{\xi}=A \xi$, if the matrix A is stable, $K_{D}$ and $K_{P}$ were suitably chosen.

\subsection{Ground motion database}

Nowadays an earthquake ground motion recording and registration is a tool for several studies in different areas; Berkeley University have a web database for Pacific Earthquake Engineering Research center (PEER ) with the objective of create an electronic library of recorded ground motion acceleration time series suitable for use by engineering practitioners for time-history dynamic analyses of various facility types.

PEER took great effort in ensuring that all the data had been processed consistently and that it was reliable in all regards. PEER also gathered related meta-data such as earthquake magnitude, various site-to-source distance measures, style of faulting, local site conditions at the recording stations, and other relevant engineering parameters. Products of this electronic library project include an internet web-based application that includes the original unscaled PEER ground motion database, inclusive of acceleration time series and corresponding acceleration response spectra, and a feature based on an improved Directed Graph Markup Language (DGML ) software tool that allows the user to select, scale, and evaluate time series for applications [10].

Several features of the PEER Ground Motion Database are highlighted as follows [11]:

- Graphic User Interface (GUI ). GUI provides a user-friendly interface for data input and processing. The users operation involves checking boxes, selecting pop-up menus, and pushing buttons. There are numerous features that are designed to facilitate easy usage. One example is the software can automatically retrieve previously specified input data. 
- Interactive Plotting. Results in each step can be visualized in real time, and results from different sets of input parameters can be easily compared. The software provides a list of the selected records with important information. By clicking the list, users can hyphenate inspect the response spectrum, acceleration/velocity displacement time-series of each individual record for each component.

- Flexibility. The PEER Ground Motion Database (PGMD ) provides users flexibility to exercise different criteria to select the design records. Users have the options to scale or not to scale the records; to select the record according to the geometric mean of fault normal and fault parallel components. The user can choose to select records with pulses or without pulses. The number of output records can be user-specified, and users have the capacity to manually select and adjust the results to meet their specific requirement.

- Easy Output.The PGMD provides easy ways to output search results, plots and tables. The web based application can automatically generate a "Target Spectrum Report" and a "Search Result Report" and save them into a Microsoft Excel spreadsheet file. The PGMD reports summarize search criteria, scale factors, scaled spectra of selected records, and other record information. The response spectra plots and time series plots can be exported into figure files of different formats. Finally, files containing acceleration time series of selected records can be saved for each project.

- Efficiency. The algorithm of the PGMD web application is robust and efficient. The search engine can scan and sort the Next Generation of Ground-Motion Attenuation Models (NGA) database within a few seconds.

- Extendibility. The PGMD web application is directly connected to the NGA flatfile and strong motion database, so it can be easily upgraded to accommodate future development of the NGA database.

\subsubsection{Earthquake ground motion profile}

Ground motion database is a powerful tool in order to obtain earthquake ground motion "profiles" using an interactive web based application that allows the user to select sets of strong ground motion acceleration time series that are representative of design ground motions. The user specifies the design ground motions in terms of a target response spectrum and the desired characteristics of the earthquake ground motions in terms of earthquake magnitude, source-tosite distance and other general characteristics [10]. 



\section{CIDESI Hexapod HxCf1 Project}

This chapter presents an overview of the CIDESI Hexapod project since its proposal, explaining the different phases of development and the previous work that has been done by members of the High Reliability Real-Time Control Systems (HRRTCS) at CIDESI in order to make possible the development of this master thesis project.

\subsection{Project overview}

The CIDESI Hexapod HxCf1 robotic system is a project that aims to develop an industrial grade robotic system that provides similar functionalities to the ones found in a common used industrial robot. The Hexapod robotic system project consists of the parallel robot, the controller, Human Machine Interface (HMI ), and a user documentation; all these as the basic components that are generally included in a commercial robotic system used in the industry.

The CIDESI Hexapod project aims is high and in order to achieve the desired result, its development is divided in four phases, as shown in Table 3-1.

\begin{tabular}{|c|c|c|c|c|}
\hline Parameter & Phase I & Phase II & Phase III & Phase IV \\
\hline Accuracy & $1 \mathrm{~mm}$ & $100 \mathrm{um}$ & $1 \mathrm{um}$ & \\
\hline Payload & $10 \mathrm{~kg}$ & $100 \mathrm{~kg}$ & $500 \mathrm{~kg}$ & \\
\hline Actuator & $\begin{array}{l}\text { LACTP6P- } \\
12 \mathrm{~V}-20\end{array}$ & To be decided & $\begin{array}{l}\text { Linear actua- } \\
\text { tor }\end{array}$ & \\
\hline Estimated cost & $<80,000$ & $<300,000$ & $<900,000$ & \\
\hline Objective & $\begin{array}{l}\text { Experimental } \\
\text { prototype }\end{array}$ & $\begin{array}{l}\text { Low capabili- } \\
\text { ties app. }\end{array}$ & Normal app. & $\begin{array}{l}\text { High capabili- } \\
\text { ties app. }\end{array}$ \\
\hline
\end{tabular}

Table 3-1: Development phases for the CIDESI Hexapod project. Taken from [21] 
The project is currently in the Phase I, where the robot is mainly intended as an experimental prototype; this prototype consists of detailed mechanical design, hardware elements of the controller and the controller software of the robot as the components that already been developed and work together in order to have a fully functional SP prototype.

Future developments of the system, consist in replace commercial actuators Glideforce LACTP6P12V-20 with CIDESI own linear actuators, as well as the development of a Teach Pendant for the robot.

\subsection{Previous work on the CIDESI Hexapod HxCf1 project}

This section describes the research and development work that was done on the CIDESI Hexapod system since its proposal until the Phase I of the project.

\subsubsection{Industrial Robot project proposal}

In 2012, Gengis K. Toledo-Ramirez proposed the design of a six DOF industrial robot at CIDESI in the research center located in Nuevo León, México. The main goal of the proposal was to aid national industry with the reduction of manufacturing costs by increasing the quality and efficiency.

In 2015 a new project proposal was done, this time was a research project called "Development of technologies for a Stewart-Gough Platform". The main goal was to develop a robotic platform at an experimental level with enough efficiency to be of interest to the research, academic and industrial sectors; the project was proposed and to be developed by the Research and Development group of the Automated Systems Division at CIDESI located in the state of Querétaro, México [21].

\subsubsection{Demonstration development for a Hexapod robot}

The main activity of this work was to develop the programming of three demo programs for the commercial parallel robot FANUC F-200ib. For the first program, the objective was to visualize the maximal positions of displacement $(\mathrm{x}, \mathrm{y}, \mathrm{z})$ and rotation $(\alpha, \beta, \gamma)$ of the robot, as a first demonstration. In the second program, it is shown how to program the robot in order to 
perform industrial tasks, using simulated digital inputs. Finally, the third program consisted in the communication between a robot and an industrial vision system [34].

\subsubsection{Hexapod system for truck motion simulation}

In 2016, Víctor David Acosta-Abraham developed a study and preliminary draft for a truck simulator mechanism [12]. The mechanism is based on a SP and its purpose is to create an environment where the forces to which a truck is subjected when operating are simulated. Using servo-motors and Arduino, a prototype was developed and a Labview interface was created in order to solve the inverse kinematic equations and establish communication between the components to drive the servo-motors.

\subsubsection{Alpha prototype of an Hexapod robot}

Gerson Andrés Díaz López [29] developed an alpha concept design for the Hexapod system. This concept design consist on preliminary ideas for the mechanical structure of the robot, the control system and communication possibilities for the system.

\subsubsection{Preliminary Design of a Stewart-Gough platform}

The preliminay design of the mechanical structure of the CIDESI Hexapod system was developed by Rubén Ordaz Madrigal in 2017 [33]. In this work, the commercial mechanical components as joints and actuators were selected and a preliminary design was developed using these components.

\subsubsection{Detailed Design of a Stewart-Gough platform}

Based on the previous work done by Ordaz and guided by area director, Noé Reyes, the detailed design for the CIDESI Hexapod was developed by José-Ramón Barajas-Fletes [27]. In this detailed design, some parts were changed, and the Computer Aided Design (CAD ) model was refined. Additional to the model, motion simulations were performed in order to determine the workspace capabilities of the Hexapod. 


\subsubsection{Robot assembly}

Based on the detailed design CAD model, the robot components were fabricated and the selected commercial components were acquired. Once all components were available, the robot was assembled.

First, the parts that form the universal joints were assembled and fixed to the base platform of the robot. Then, the linear actuators were assembled with the universal joints and secured in place, adding the head couplings on the top of the shaft of the actuators. After this, the spherical joints were screwed in the mobile platform,secured with bolts and the coupling disc was assembled to the mobile platform. Finally, all the actuators were assembled to the spherical joints and secured in place with screws. The assembled robot highlighting the main components is shown in Figure 3-1 [21].

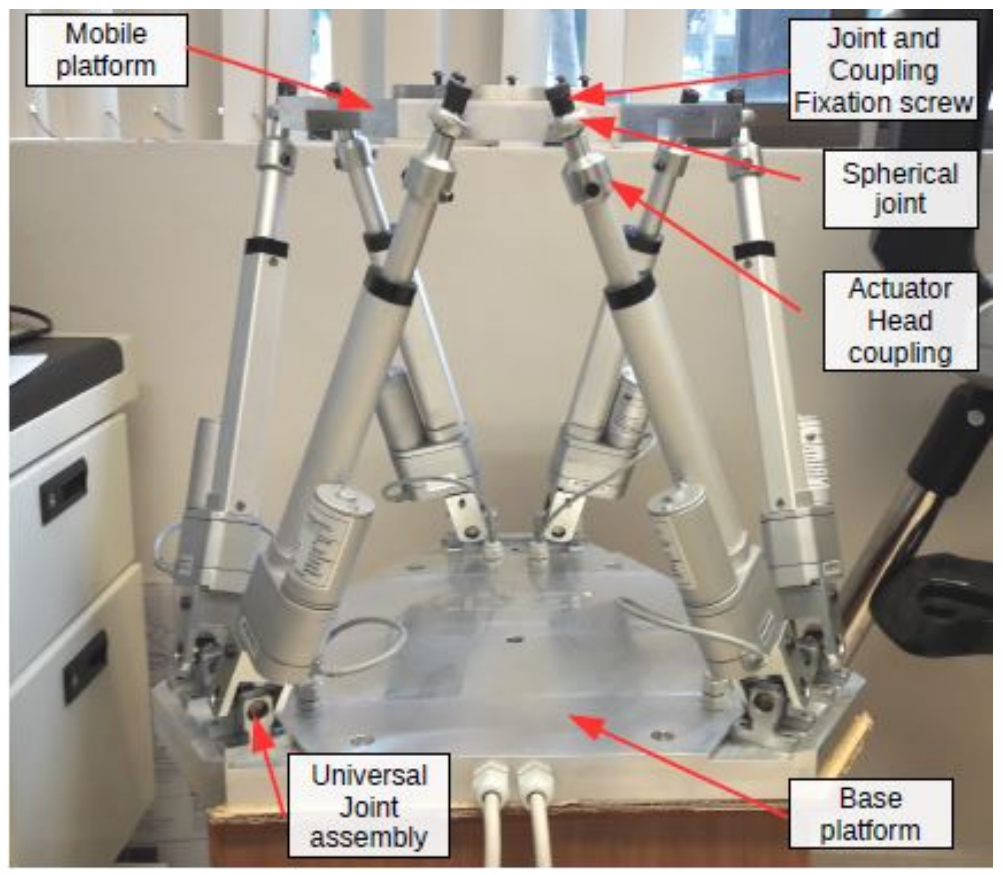

Figure 3-1: Assembled CIDESI Hexapod system. Taken from [21].

\subsubsection{Robot Controller}

This section describes a general overview of the implemented hardware and software components of the Hexapod HxCf1 robot controller. 


\subsubsection{Hardware}

The hardware components of the controller consist in DC power supplies, protoboards, jrk drivers and its related connection with the power supply and the robot itself.

The base of the prototype is a plastic plate where all components are fixed. This material was used because unlike wood, acrylic, or metal, it is easy and fast to make fixation points for the components. For the connection of the power supply channels, the communication lines of the jrk drivers, and the AND gate circuit, three protoboards were used (see Figure 3-2).

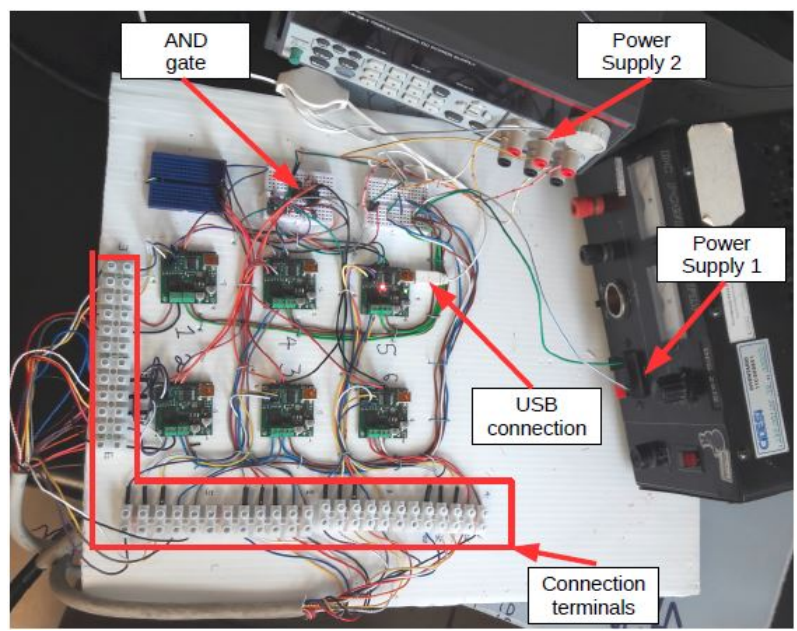

Figure 3-2: Assembly of critical components of the CIDESI Hexapod controller. Taken from [21].

Every actuator requires five wires, which must come from the jrk drivers in the controller board. The controller board and the robot are joint together by two 20-wire cables using six 12-point terminals: three on the robot side, and three on the controller side. This provides 36 connections, but only 30 are needed for the six actuators; the remaining connections can be used for other purposes in future development of the system.

The bottom part of the robot's base frame is designed to contain the electrical connections that will go from the actuators to the controller board. In order to connect the actuators to the 20wire cables, three 12-point terminals were used, as shown in Figure 3-3. One cable connects actuators 1, 2, and 3, and the other cable connects actuators 4, 5, and 6. The remaining cables were not removed because they may be used in future development of the system. 


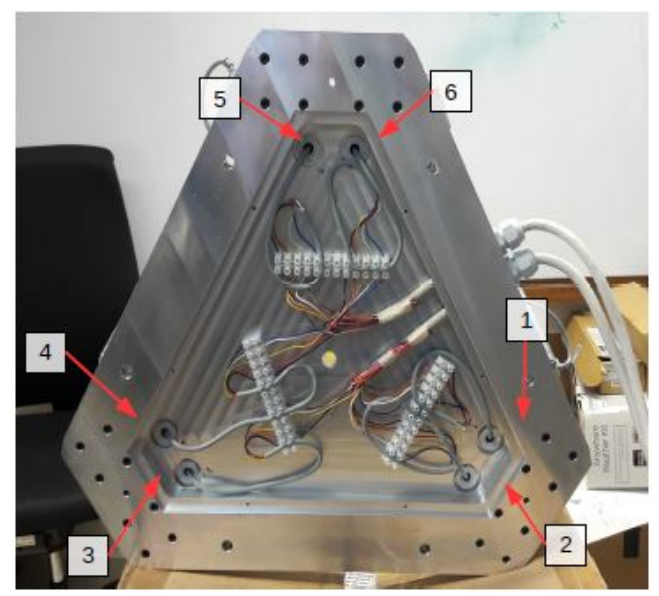

Figure 3-3: Robot electrical connections. Taken from [21].

\subsubsection{Software}

The software part of the controller was implemented as modules; these modules were implemented as $\mathrm{C}++$ classes, whose internal parameters can be read from a configuration file and initialized inside a class constructor. Communication between classes is achieved by allowing class methods to call other methods from other classes [21].

Additionally, there are other software modules which only contain helper functions, such as those needed for parsing strings, or logging events and errors in a log file. The functions provided by these modules are available to all classes.

Some of the controller's software modules require the use of advanced mathematical functions, such as matrix multiplication, norm and vector product. While there exist libraries for $\mathrm{C}++$ that provide functions to perform these operations, their use is somewhat difficult, and their syntax is complicated [21].

MatLab has a tool called Coder, which allows the generation of $\mathrm{C} / \mathrm{C}++$ source code from standard .m files. Coder takes a matlab function as input and produces a header file with the function declaration, a source file with the function definition, a sample test program, and other header files with data types definitions, for use in different platforms.

After the implementation of the modules, the robot can be manipulated using Command Line Interface (CLI) where the user just need to write a command using specific input values that are described in the user documentation manual. The robot can move in translational and rotational axis, the actuators can be manipulated individually and it can run predefined routines using specific input coordinates. 


\section{CIDESI Hexapod HxCf1 characteriza- tion}

This chapter presents the development of the required equations in order to obtain a full characterization of the CIDESI HxCf1 Hexapod necessary to create and run a dynamic model.

\subsection{Inverse Kinematics}

Kinematics studies the movement of a mechanism without taking into account weights, inertia and the forces that generate it. In general, kinematic analysis of a parallel or serial manipulator can be inverse kinematics or direct kinematics, both of them with advantages and withdraws.

Inverse kinematics calculate the actuator lengths, using as input, the position and orientation of the final effector, which can be described by generalized coordinates; these coordinates are usually established at a specific point in the end effector. Inverse kinematics has an unique solution for parallel manipulators, due to its ease of calculation compared to direct kinematics and that is why this is going to be used in the HxCf1 CIDESI Hexapod.

A model representation of the CIDESI HxCf1 Hexapod with the coordinate axis, components and nomenclature from front and top view is shown in Figure 4-1. 


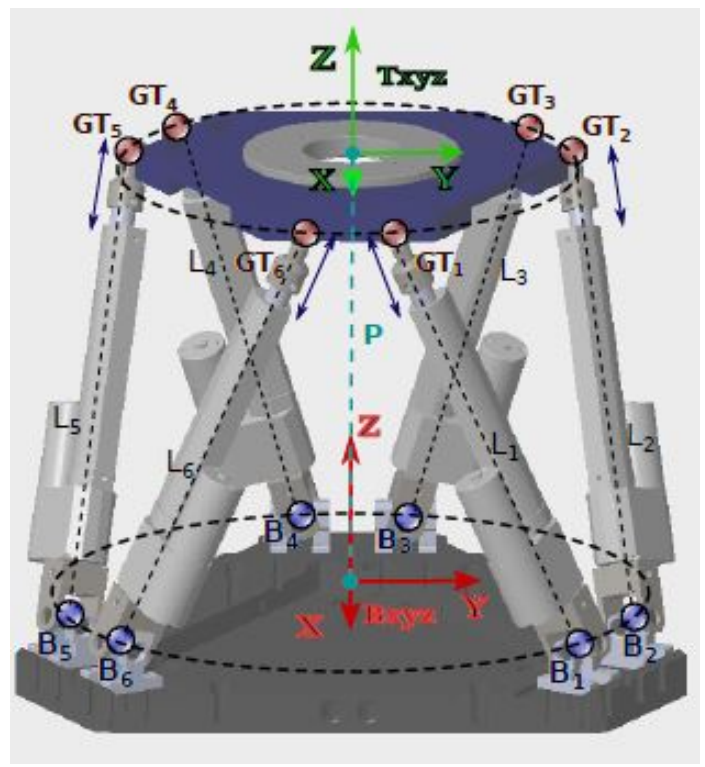

(a) Front view of HxCf1.

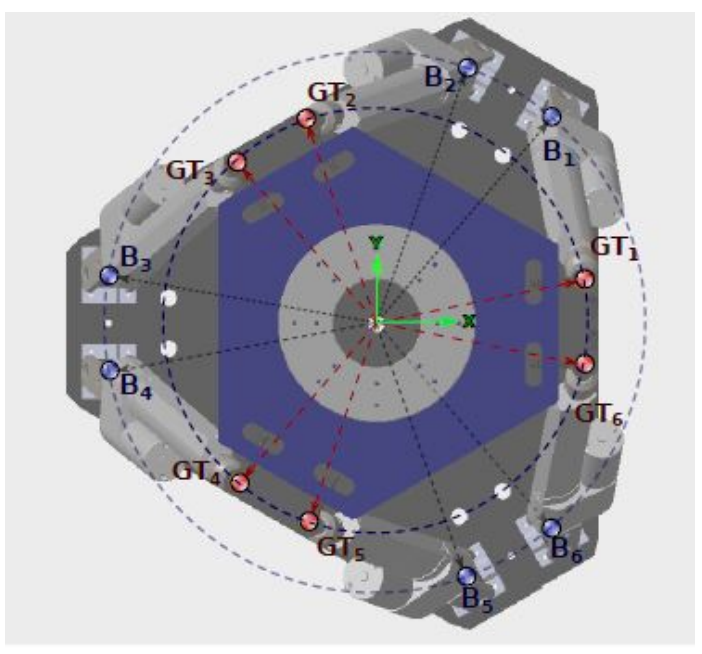

(b) Top view of HxCf1.

Figure 4-1: CIDESI HxCf1 Hexapod. Taken from [13].

\subsubsection{Base and Mobile platforms configuration}

The Figure 4-2 is a schematic representation of the mobile platform, where the coordinate system is referred as $T_{x y z}$ and $\theta_{p}$ is the angle between the end connection of each pair of actuators having a platform radius denoted as $r_{p}$.

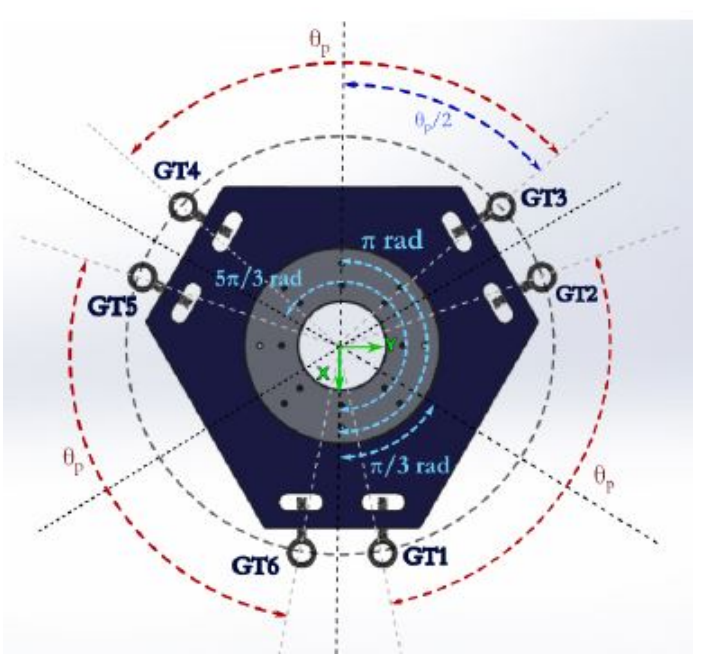

Figure 4-2: Angle characterization for the mobile platform using its coordinate system $T_{x y z}$. Taken from [13]. 
The Figure 4-3 show the characterization of points $G T_{i}$, where $i$ represent the number of each actuator and values for $\lambda_{1}$ to $\lambda_{6}$ is obtained using $\theta_{p}$ as a known value.

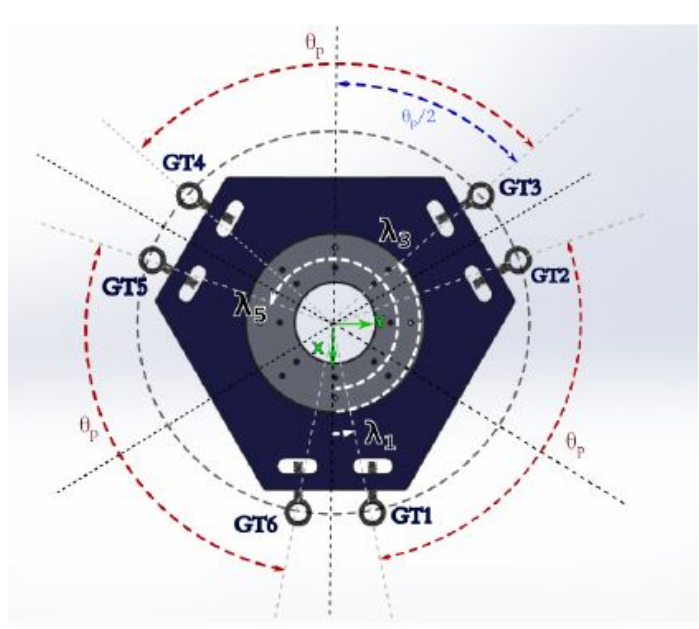

(a) Schematic representation for $\lambda_{1}, \lambda_{3}$ and $\lambda_{5}$.

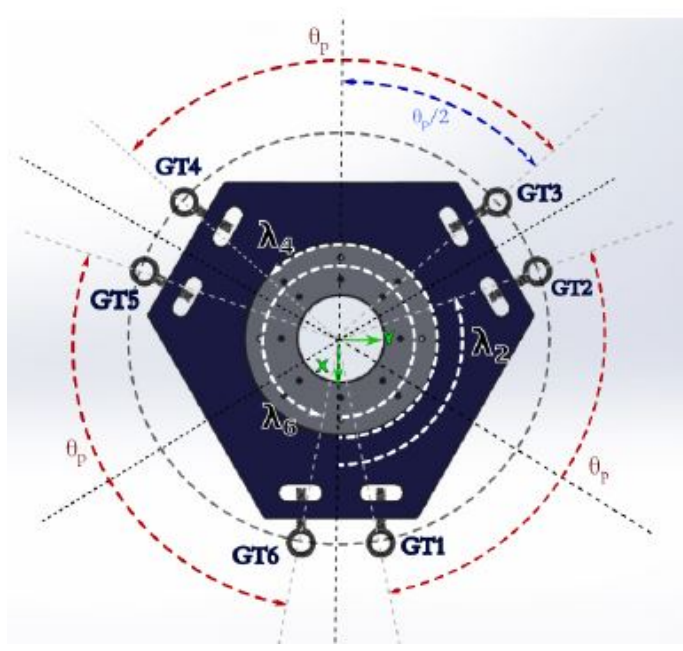

(b) Schematic representation for $\lambda_{2}, \lambda_{4}$ and $\lambda_{6}$.

Figure 4-3: Schematic representation of the mobile platform. Taken from [13].

The Figure 4-4 is a schematic representation of the base platform, where the coordinate system is referred as $B_{x y z}$ and $\theta_{b}$ is the angle between the base connection of each pair of actuators having a platform radius denoted as $r_{b}$.

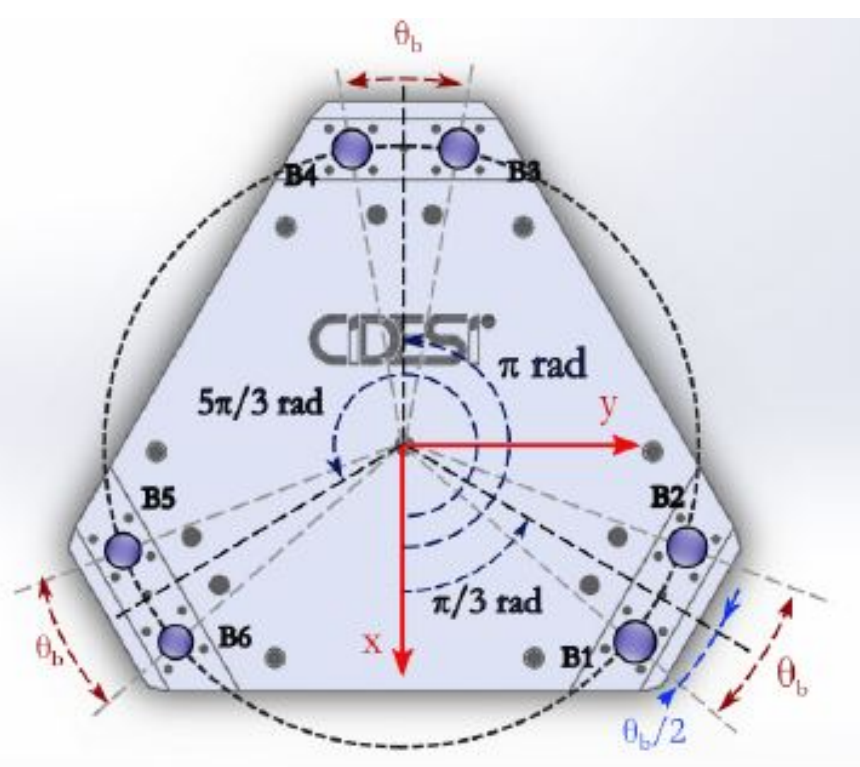

Figure 4-4: Angle characterization for the base platform using its coordinate system $B_{x y z}$. Taken from [13]. 
The Figure 4-5 show the characterization of points $B_{i}$, where $i$ represent the number of each actuator and values for $v_{1}$ to $v_{6}$ is obtained using $\theta_{b}$ as a known value.

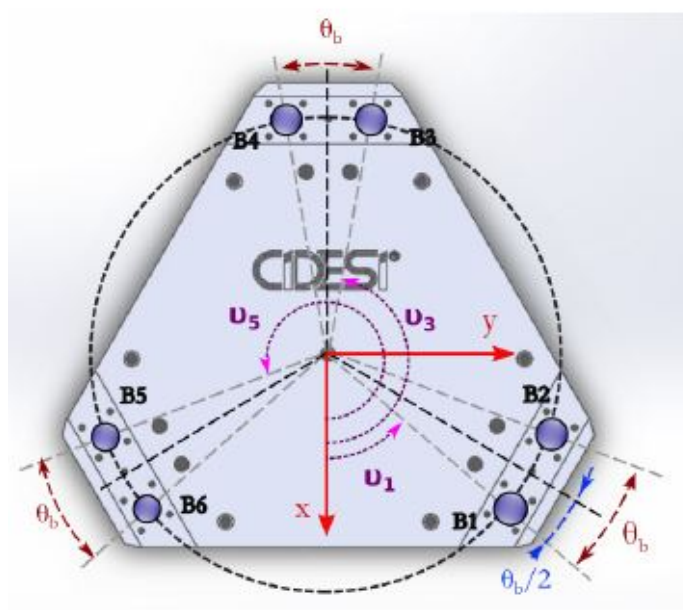

(a) Schematic representation for $v_{1}, v_{3}$ and $v_{5}$.

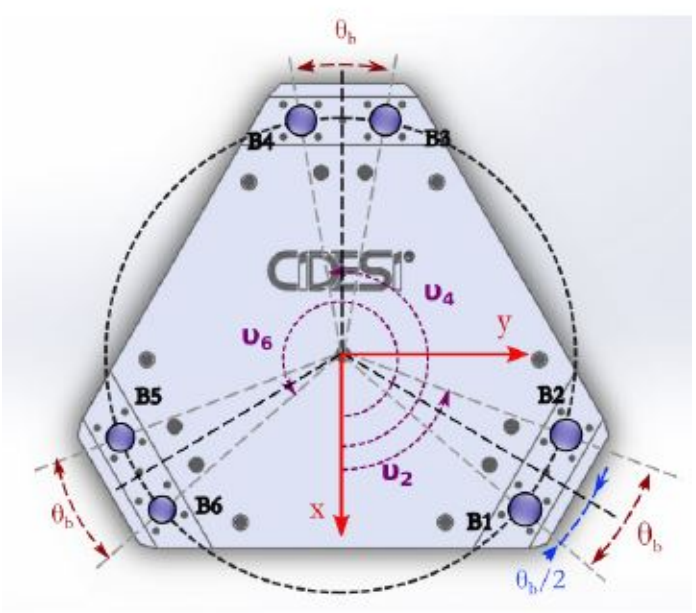

(b) Schematic representation for $v_{2}, v_{4}$ and $v_{6}$.

Figure 4-5: Schematic representation of the base platform. Taken from [13].

The parameters to take into account in order to define the Hexapod base and mobile platform configuration are shown in Table 4-2.

\begin{tabular}{lcc}
\hline Parameter & Symbol & Value \\
\hline Base platform radius & $r_{b}$ & $192.86 \mathrm{~mm}$ \\
Mobile platform radius & $r_{p}$ & $247.97 \mathrm{~mm}$ \\
Angle $\theta_{b}$ & $\theta_{b}$ & $20.4414^{\circ}$ \\
Angle $\theta_{p}$ & $\theta_{p}$ & $97.88^{\circ}$
\end{tabular}

Table 4-1: Physical parameters of HxCf1 Hexapod. Taken from [13]

Using the mobile platform configuration and the parameters shown in Table 4-2, the following equation corresponds to the calculation of the points $G T_{i}$ :

$$
G T_{i}=\left[\begin{array}{c}
G T_{x i} \\
G T_{y i} \\
G T_{z i}
\end{array}\right]=\left[\begin{array}{c}
r_{p} \cos \left(\lambda_{i}\right) \\
r_{p} \sin \left(\lambda_{i}\right) \\
0
\end{array}\right]
$$

with 


$$
\lambda_{i}=\frac{i \pi}{3}-\frac{\theta_{p}}{2} \quad \text { for } \quad i=1,3,5
$$

and

$$
\lambda_{i}=\lambda_{i-1}+\theta_{p} \quad \text { for } \quad i=2,4,6
$$

Where $G T_{i}$ is the position vector from the union points of the mobile platform with each one of the six actuators taking as base the coordinate system $T_{x y z}$ using the trigonometric functions that apply for each actuator depending if it is even or odd. For the base platform the same procedure is done, just adjusting the value of the corresponding parameters as it was mentioned in Figure 4-2, Figure 4-3 and Table 4-2.

$$
B_{i}=\left[\begin{array}{c}
B_{x i} \\
B_{y i} \\
B_{z i}
\end{array}\right]=\left[\begin{array}{c}
r_{b} \cos \left(v_{i}\right) \\
r_{b} \sin \left(v_{i}\right) \\
0
\end{array}\right]
$$

with

$$
v_{i}=\frac{i \pi}{3}-\frac{\theta_{b}}{2} \quad \text { for } \quad i=1,3,5
$$

and

$$
v_{i}=\lambda_{i-1}+\theta_{b} \quad \text { for } \quad i=2,4,6
$$

The coordinate values for each point calculated using Equation 4.1 and Equation 4.2 are shown in Table 4-2.

\begin{tabular}{lccc}
\hline Point & Coordinates & Point & Coordinates \\
\hline B1 & $(160.1226,189.3407,0)$ & GT1 & $(189.2780,36.9977,0)$ \\
B2 & $(83.9125,189.3407,0)$ & GT2 & $(-62.5981,182.4184,0)$ \\
B3 & $(-244.0351,43.9999,0)$ & GT3 & $(-126.6799,145.4207,0)$ \\
B4 & $(-244.0351,-43.9999,0)$ & GT4 & $(-126.6799,-145.4207,0)$ \\
B5 & $(83.9125,-233.3407,0)$ & GT5 & $(-62.5981,-182.4184,0)$ \\
B6 & $(160.1226,-189.3407,0)$ & GT6 & $(189.2780,-36.9977,0)$
\end{tabular}

Table 4-2: Point coordinates for $B_{i}$ and $G T_{i}$. Taken from [13] 


\subsubsection{Mobile platform position and orientation}

The mobile platform is a rigid body that will be subjected to movement in a three-dimensional space that will be measured through a point of origin in the same platform, that is, $(x, y, z) \in \mathbb{R}$

The mobile platform positioning is described by a position vector named $\mathrm{P}$, located at the origin of the coordinate system of the mobile platform with respect to the origin of the coordinate system of the base platform, and a rotation matrix ${ }^{B} R_{T}$, which is nesting the absolute angles $(\alpha, \beta, \gamma)$ in the coordinate system of the mobile platform.

Angle $\alpha$ represent the rotation with respect to the $\mathrm{x}$ axis, $\beta$ represent the rotation with respect to the $\mathrm{y}$ axis and $\gamma$ represent the rotation with respect to the $\mathrm{z}$ axis. The corresponding rotation matrices $R_{x}(\alpha), R_{y}(\beta)$ and $R_{z}(\gamma)$ are given by:

$$
\begin{aligned}
R_{X}(\alpha) & =\left[\begin{array}{ccc}
1 & 0 & 0 \\
0 & \cos \alpha & -\sin \alpha \\
0 & \sin \alpha & \cos \alpha
\end{array}\right] \\
R_{Y}(\beta) & =\left[\begin{array}{ccc}
\cos \beta & 0 & \sin \beta \\
0 & 1 & 0 \\
-\sin \beta & 0 & \cos \beta
\end{array}\right] \\
R_{Z}(\gamma) & =\left[\begin{array}{ccc}
\cos \gamma & -\sin \gamma & 0 \\
\sin \gamma & \cos \gamma & 0 \\
0 & 0 & 1
\end{array}\right]
\end{aligned}
$$

There are twelve different combinations for the order of rotation of this angles, considering also combinations of three rotations with respect to only two axes, that can represent arbitary 3D rotations. In this document we adopt the order $\alpha, \beta, \gamma$ all of them w.r.t. the fixed platform, that is:

$$
\begin{gathered}
{ }^{B} R_{T}=R_{Z}(\gamma) R_{Y}(\gamma) R_{X}(\alpha) \\
=\left[\begin{array}{ccc}
\cos \beta \cos \gamma & \cos \gamma \sin \alpha \sin \beta-\cos \alpha \sin \gamma & \sin \alpha \sin \gamma+\cos \alpha \cos \gamma \sin \beta \\
\cos \beta \cos \gamma & \cos \alpha \cos \gamma+\sin \alpha \sin \beta \sin \gamma & \cos \alpha \sin \beta \sin \gamma-\cos \gamma \sin \alpha \\
-\sin \beta & \cos \beta \sin \alpha & \cos \alpha \cos \beta
\end{array}\right]
\end{gathered}
$$


Thus, the vector $L_{i}$ corresponding to the link $i$ is obtained as follows (in $B_{X Y Z}$ coordinates).

$$
L_{i}=R_{X Y Z} G T_{i}+P-B_{i}, i=1, \ldots, 6
$$

that is,

$$
L_{i}=\left[\begin{array}{c}
r_{p}[\sin (\alpha) \sin (\beta) \cos (\gamma)-\cos (\alpha) \sin (\gamma)] \sin \left(\lambda_{i}\right)+r_{p} \cos (\beta) \cos (\gamma) \cos \left(\lambda_{i}\right)+x-r_{b} \cos \left(v_{i}\right) \\
r_{p}[\sin (\alpha) \sin (\beta) \sin (\gamma)+\cos (\alpha) \cos (\gamma)] \sin \left(\lambda_{i}\right)+r_{p} \cos (\beta) \sin (\gamma) \cos \left(\lambda_{i}\right)+y-r_{b} \sin \left(v_{i}\right) \\
r_{p} \operatorname{coa}(\alpha) \cos (\beta) \sin \left(\lambda_{i}\right)-r_{p} \sin (\beta) \cos \left(\lambda_{i}\right)+z
\end{array}\right]
$$

and their lengths, $l_{i}$, correspond to the actuator positions

$$
l_{i}=\left\|L_{i}\right\|
$$

\subsection{Jacobian}

To analyse a mechanical and articulated system, an analysis is required to perform linear and angular velocity calculations. For the Stewart Gough platform, these cinematic velocities will be obtained using the Jacobian matrix; the speed relationships between the positions and orientations of the mobile platform are determined by the Jacobian.

The Jacobian is one of the most important variables in the analysis and control of robot movements. This arises virtually in every aspect of robotic manipulation: in the study and execution of smooth trajectories, in the determination of singular positions, in the execution of anthropomorphic movement, in the emergence of dynamic equations of movement and in the transformation of forces and torques from the final effector to the manipulator joints. The Jacobian is usually represented as a vector notation, expressed as the following equation:

$$
\dot{L}=J \dot{X}
$$

Where $\dot{L}$ are the actuator velocities and $\dot{X}$ is the velocity of the coordinates of the mobile platform $\left(\dot{X}_{p-0}\right)$. There are several forms to work with the velocity relationships in parallel robots, but in this work is going to be used the proposal that describes the Jacobian as: 


$$
J_{B}=J_{I B} J_{I I B}
$$

With the vector $G T_{i}=\left[\begin{array}{lll}G T_{x i} & G T_{y i} & G T_{z i}\end{array}\right]^{T}$ obtained, the $T_{j}$ point in the mobile platform with reference on the coordinate system $B_{x y z}$ is obtained by:

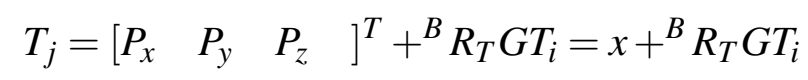

The velocity in the joint point $T_{j}$ is obtained by a differentiation of the Equation 4.10 with respect of time:

$$
\overrightarrow{V_{T j}}=\left[\begin{array}{lll}
\dot{P}_{x} & \dot{P}_{y} & \dot{P}_{z}
\end{array}\right]^{T}+\omega \times \quad{ }^{B} R_{T} G T_{i}=\dot{x}+\omega \times \quad{ }^{B} R_{T} G T_{i}
$$

Where $\omega=\left(\omega_{x}, \omega_{y}, \omega_{z}\right)$ is the angular velocity of the mobile platform with respect to the base platform.

$$
\omega=\left[\begin{array}{c}
\dot{\alpha} \cos \beta \cos \gamma-\dot{\beta} \sin \gamma \\
\dot{\alpha} \cos \beta \sin \gamma-\dot{\beta} \cos \gamma \\
\dot{\alpha} \sin \beta-\dot{\gamma}
\end{array}\right]=\left[\begin{array}{ccc}
\cos \beta \cos \gamma & -\sin \gamma & 0 \\
\cos \beta \sin \gamma & \cos \gamma & 0 \\
\sin \beta & 0 & -1
\end{array}\right]\left[\begin{array}{c}
\dot{\alpha} \\
\dot{\beta} \\
\dot{\gamma}
\end{array}\right]
$$

The velocity vector defined as $\dot{T}_{j}$ in the contact point with the linear actuators $i$ is generated by the action of these, and due to the contact without deformation between mobile platform and the actuators, the velocity between them is related as follows:

$$
\dot{L_{i}}=\overrightarrow{V_{T j}} \vec{u}_{i}=\left[\begin{array}{lll}
\dot{P}_{x} & \dot{P}_{y} & \dot{P}_{z}
\end{array}\right]^{T} * \vec{u}_{i}+\omega \times \quad{ }^{B} R_{T} G T_{i} * \vec{u}_{i}=\dot{x} * \vec{u}_{i}+\omega \times \quad{ }^{B} R_{T} G T_{i} * \vec{u}_{i}
$$

Where $u_{i}$ is the unitary vector along the linear actuator and result of the velocity of the same; the value is obtained as follows:

$$
u_{i}=\frac{L_{i}}{\left\|L_{i}\right\|}
$$

Using Equation 4.12, the actuator velocities are expressed in matrix form and the following equation is obtained: 


$$
\dot{L}=J_{B}\left[\begin{array}{c}
\dot{x} \\
\omega
\end{array}\right]=J_{I B} J_{I I B} \dot{X}_{p-0}
$$

Where the first Jacobian matrix is:

$$
J_{I B}=\left[\begin{array}{cccc}
u_{x 1} & u_{y 1} & u_{z 1} & \left({ }^{B} R_{T} G T_{1} \times \vec{u}_{1}\right)^{T} \\
u_{x 2} & u_{y 2} & u_{z 2} & \left({ }^{B} R_{T} G T_{2} \times \vec{u}_{2}\right)^{T} \\
u_{x 3} & u_{y 3} & u_{z 3} & \left({ }^{B} R_{T} G T_{3} \times \vec{u}_{3}\right)^{T} \\
u_{x 4} & u_{y 4} & u_{z 4} & \left({ }^{B} R_{T} G T_{4} \times \vec{u}_{4}\right)^{T} \\
u_{x 5} & u_{y 5} & u_{z 5} & \left({ }^{B} R_{T} G T_{5} \times \vec{u}_{5}\right)^{T} \\
u_{x 6} & u_{y 6} & u_{z 6} & \left({ }^{B} R_{T} G T_{6} \times \vec{u}_{6}\right)^{T}
\end{array}\right]_{6 \times 6}
$$

And the second Jacobian matrix is:

$$
J_{I I B}=\left[\begin{array}{cccccc}
1 & 0 & 0 & 0 & 0 & 0 \\
0 & 1 & 0 & 0 & 0 & 0 \\
0 & 0 & 1 & 0 & 0 & 0 \\
0 & 0 & 0 & \cos \beta \cos \gamma & -\sin \gamma & 0 \\
0 & 0 & 0 & \cos \beta \sin \gamma & \cos \gamma & 0 \\
0 & 0 & 0 & \sin \beta & 0 & -1
\end{array}\right]_{6 \times 6}
$$

Using this calculations as base, it is possible to determine if the actuators are able to move from one point to another at the desired speed. If one movement require a speed higher than the maximum speed given by the actuators of the prototype $(14.6 \mathrm{~mm} / \mathrm{s})$, the movement or routine cannot be reproduced by the SP.

\subsection{Frequency work range}

As mentioned in the last section, the maximum speed of the linear actuator is $14.6 \mathrm{~mm} / \mathrm{s}$ and it is necessary to find the working frequencies of the platform that make possible the reproduction of movements. Using a sinusoidal movement, the position formula is given as follows:

$$
l=A * \sin (\omega t)
$$

Deriving the Equation 4.18 we obtain the following speed formula: 


$$
i=A * \omega * \cos (\omega t)
$$

Substituting a given amplitude of $\mathrm{A}=1 \mathrm{~mm}$ in Equation 4.19 and having into account that the speed value $V \in[0,14.6] \mathrm{mm} / \mathrm{s}$, we obtain the maximum angular velocity value of $\omega_{\max }=$ $14.45 \mathrm{rad} / \mathrm{s}$ and a frequency value of $f=2.3 \mathrm{~Hz}$ which is represented as $l_{\max }=14.45 \mathrm{~mm} / \mathrm{s}$ (see Figure 4-6).

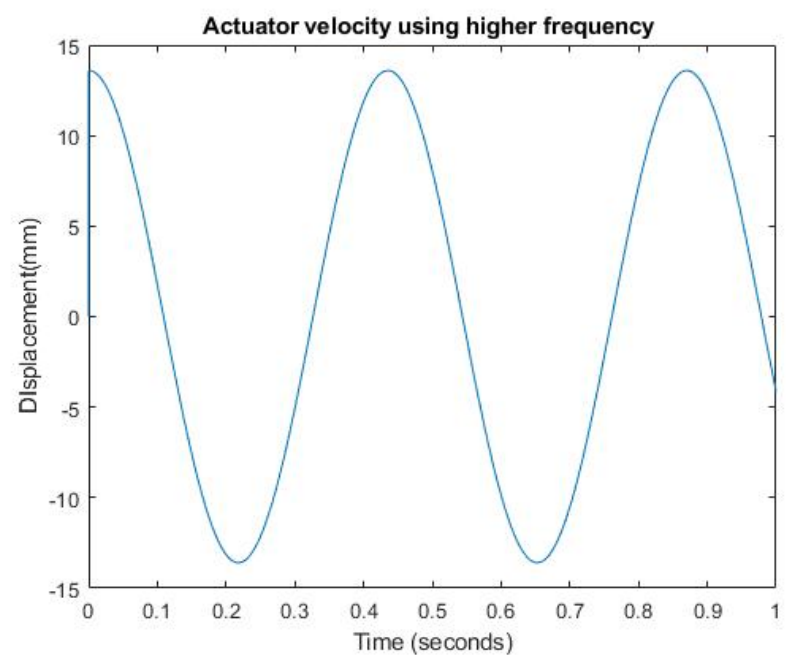

Figure 4-6: Actuator velocity using higher possible frequency.

In order to obtain the lower working frequency in particular for the $\mathrm{Z}$ axis, it is important to take into account the maximum displacement of the platform in the $\mathrm{z}$ axis; for this platform the maximum displacement is $\pm 65 \mathrm{~mm}$ from the home position. For the same sinusoidal movement the following equation for velocity is defined:

$$
\dot{z}=14.6 \mathrm{~mm} * \sin (\omega t)
$$

Integrating the Equation 4.20 we obtain:

$$
z=\frac{14.6 m m}{\omega} \cos (\omega t)
$$

Using the Equation 4.21 the minimum angular velocity value found is $\omega_{\min }=0.225 \mathrm{rad} / \mathrm{s}$ and a frequency value of $f=0.0358 \mathrm{~Hz}$ which is represented as a $l_{\min }=0.225 \mathrm{~mm} / \mathrm{s}$ (see figure Figure 4-7). 


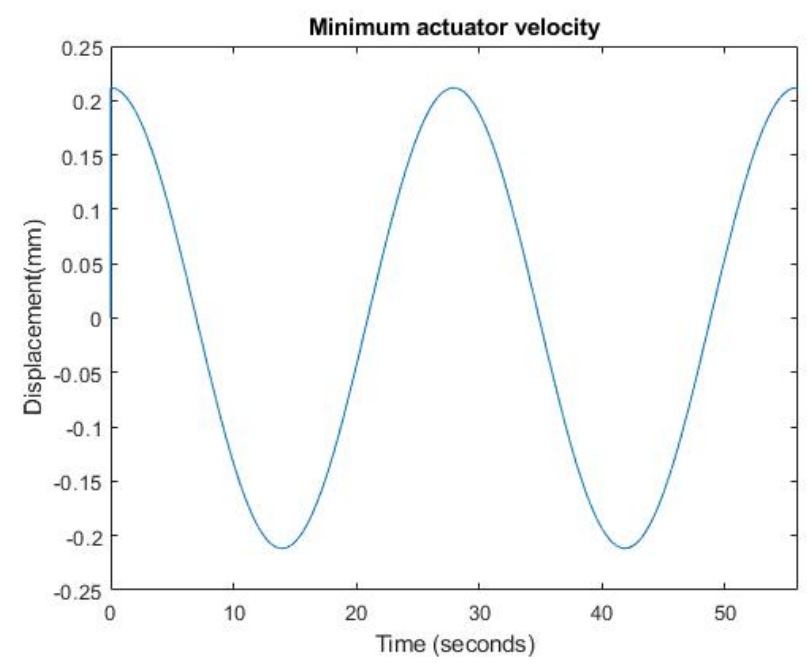

Figure 4-7: Actuator velocity using lower possible frequency. 



\section{Dynamic Model}

This chapter presents the development of the dynamic model of the CIDESI HxCf1 Hexapod using Simscape and the components required in order to perform simulations.

\subsection{Simscape dynamic model}

Dynamic of the parallel robots are more complicated in comparison with serial robots due to the existence of more than one kinematic chain, all of them connected to the mobile platform. The dynamic model of the CIDESI HxCf1 Hexapod was made taking as base the CAD model in Simscape software [13].

Simscape enables you to rapidly create models of physical systems within the Simulink environment. With Simscape, is possible to build physical component models based on physical connections that directly integrate with block diagrams and other modelling paradigms. Simscape add-on products provide more complex components such as electric motors, bridge rectifiers, hydraulic actuators, and refrigeration systems, by assembling fundamental components into a schematic and also add more analysis capabilities [40].

Simscape helps to develop control systems and test system-level performance. SimMechanics is a block diagram modeling enviroment for the engineering design and simulation of rigid body machine and their motions, using the standard Newtonian dynamics of forces and torques. With SimMechanics the user can model and simulate mechanical systems with a suite of tools to specify bodies and their mass properties, their possible motions, kinematics constraints and coordinate systems, and to initiate and measure body motions [40]. The full model with its components is seen in Figure 5-1. 


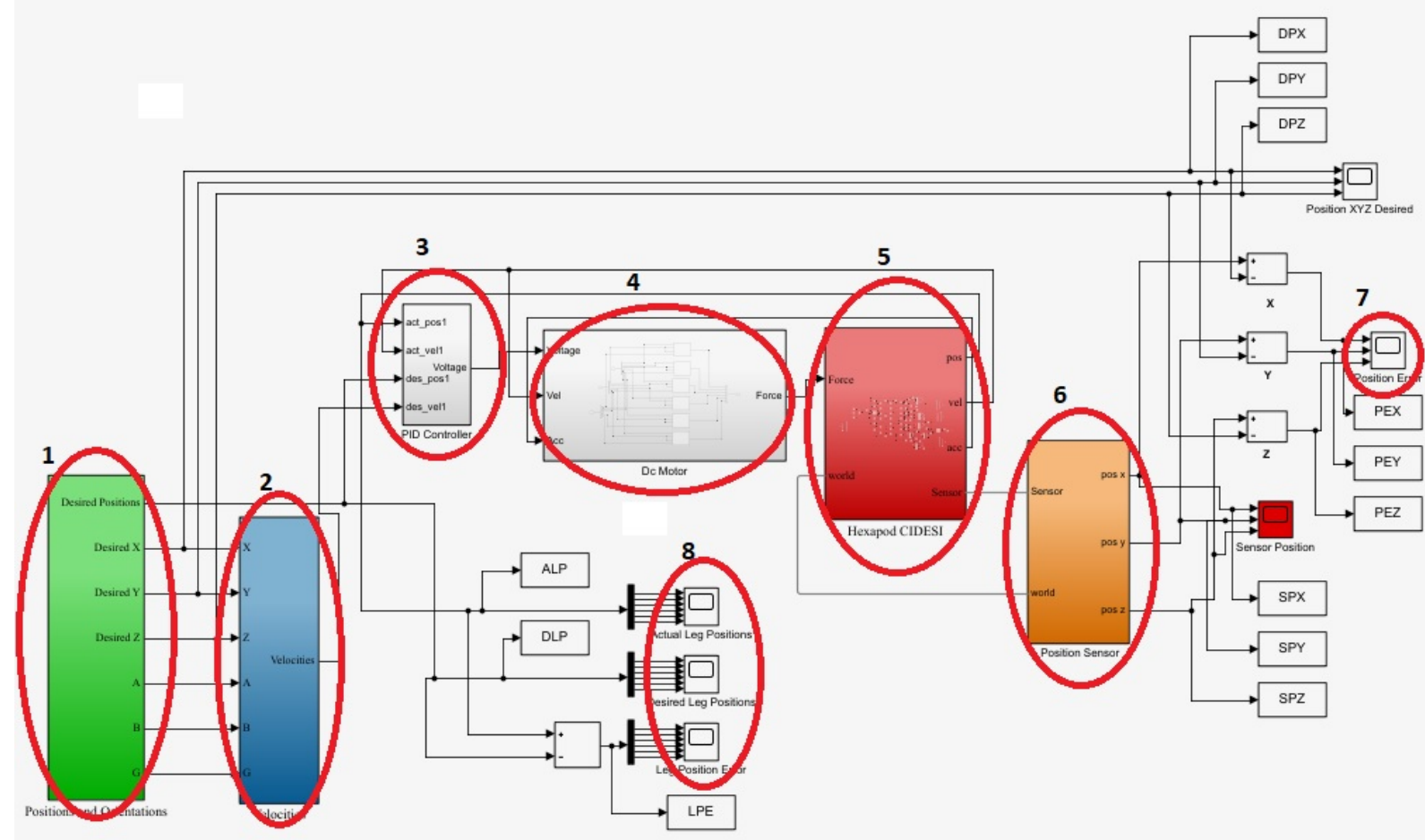

Figure 5-1: CIDESI HxCf1 Hexapod Simscape model. 1.Kinematic blocks, 2.Jacobian blocks, 3.Configurable PID controller, 4.DC motor model ,5.Assembly exported from SolidWorks, 6.Position sensor of the mobile platform, 7.Mobile platform position error, 8.Leg position position and error.

\subsubsection{CIDESI HxCf1 Hexapod Simscape model}

The CAD model of the SP was exported from SolidWorks to Simscape using SimMechanics add-on; the export procedure generates one XML file and a set of geometry files that can be imported into Simscape and converted in order to create the platform part by part by linked blocks. After the conversion is complete, the geometry blocks of the platform looks as seen in Figure 5-2 and the visual output look as seen in Figure 5-3. In order to make the generated platform the closest to the physical one, all the connections and joints were modelled and the part weights were setted to be exactly the same as the real ones. 


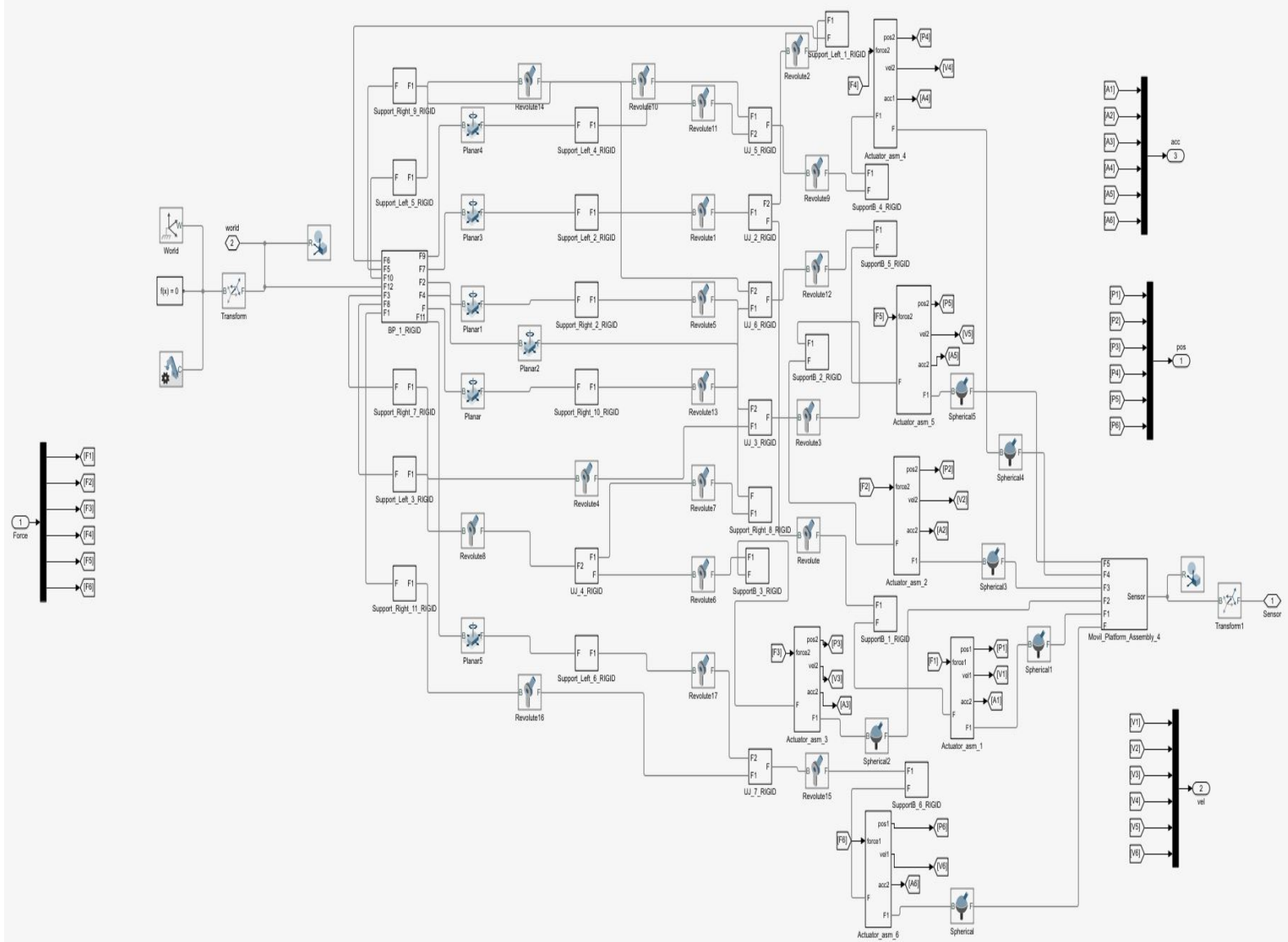

Figure 5-2: CIDESI HxCf1 Hexapod exported geometry.

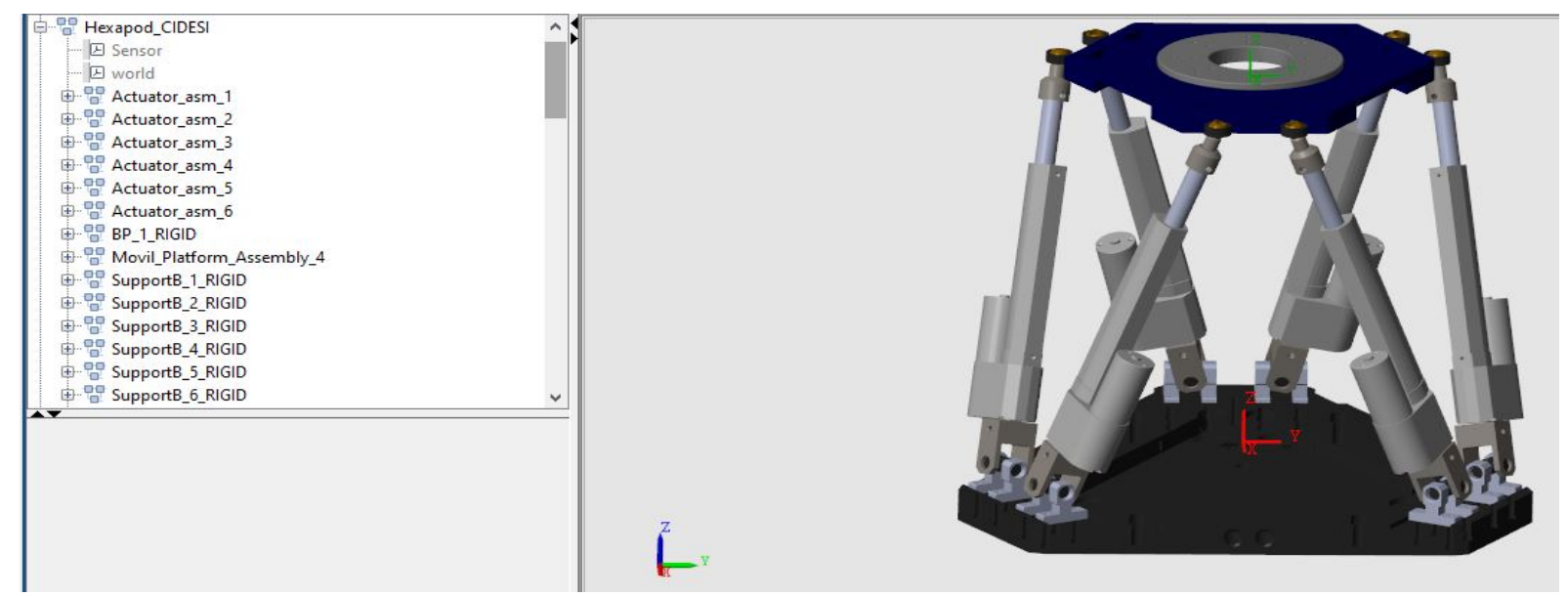

Figure 5-3: CIDESI HxCf1 Hexapod visual output. 


\subsubsection{Positions and Orientations}

The positions and orientation block consist of an input of three positions $(\mathrm{x}, \mathrm{y}, \mathrm{z})$ and three orientations $(\alpha, \beta, \gamma)$, defined by the user; these six values, are considered the desired poses that are going to be reached by the mobile platform of the hexapod. The input data, pass trough a Matlab function (see Appendix B) in order to calculate the inverse kinematics of the hexapod and obtain the leg length required for each actuator in order to reach the desired position.

\subsubsection{Velocities}

The velocities block also use the user input of three positions and three orientations and derive them in order to find the velocity in each position and orientation. This velocities pass trough another Matlab function (see Appendix C) in order to obtain the desired velocity of each actuator and use those values as an input for the configurable PID controller.

\subsubsection{Position sensor}

The position sensor is mounted on top of the mobile platform of the hexapod and measures time-dependent relationship between two frames. A Transform Sensor passively senses this 3-D time-varying transformation, and its derivatives, between the two frames.

After selecting which rotational and translational relationships, including velocities and accelerations want to measure the block displays the corresponding output physical signal ports. The sensor measures the transformation and its derivatives as follower frame relative to base frame. The transformation components can be projected into one of several frames. The output of the sensor is the $\mathrm{x}, \mathrm{y}$ and $\mathrm{z}$ position of the mobile platform that are going to be considered the real positions in order to compare them with the desired positions that were used as input and find the error.

\subsubsection{Earthquake ground motion profile}

The real earthquake ground motion data will be downloaded from the Berkeley records that are available in their own web page (peer.berkeley.edu). From the PEER Ground Motion Database the user have the option to display unscaled earthquake records as well as records that can be scaled to a target spectrum. 
For this tests, unscaled records are going to be used, having a search engine with several options in order to be more specific about the record that the user is looking for. The PGMD SEARCH ENGINE window for the unscaled section contains nine main parts, as labeled in Figure 5-4: (1) Main Search Engine; (2) Supplementary Search Engine to specify the record acceptance criteria and perform search over PEER database; (3) Spectra plotting window; (4) Ground motion record information output list; (5) Google map to display geographic location as well as basic information about each record checked; (6) Acceleration/ Velocity/ Displacement time series plotting of a selected record; (7) Zoom In time function for examining the time series of an individual record; (8) Graphic control panel for line styles and maximum number of output to be listed; (9) Buttons to save the search results and selected acceleration time series files.
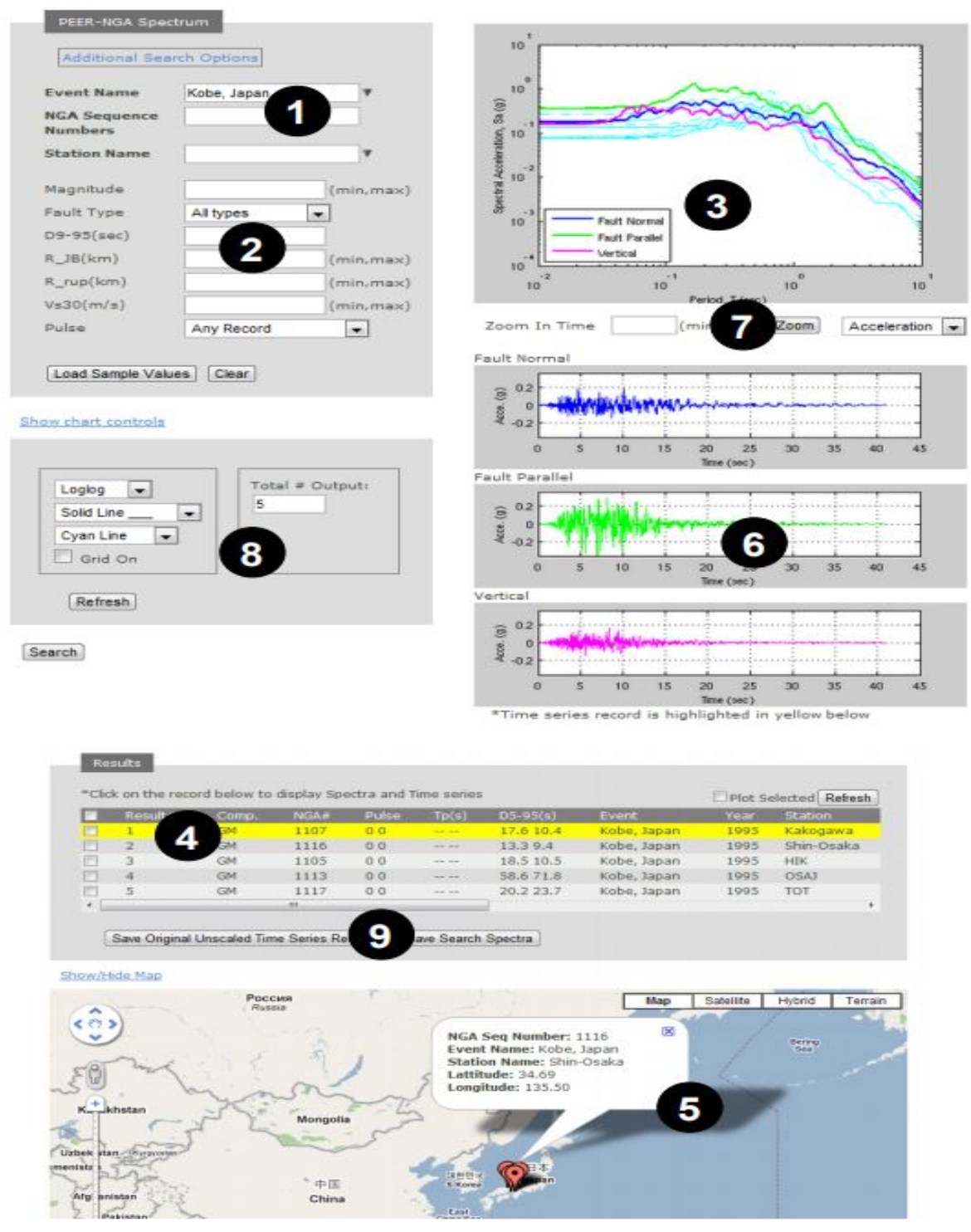

Figure 5-4: PGMD search engine interface. Taken from [11] 
In order to perform simulations with the linear actuators, glideforce LACT6P, an earthquake record with displacements in the order of millimetres was chosen. The record number of the selected earthquake is 10058 and the name of the same is Sparks, recorded in the Oklahoma station in the USA; the full information of the mentioned record as well as the peak spectra acceleration are displayed in Figure 5-5.

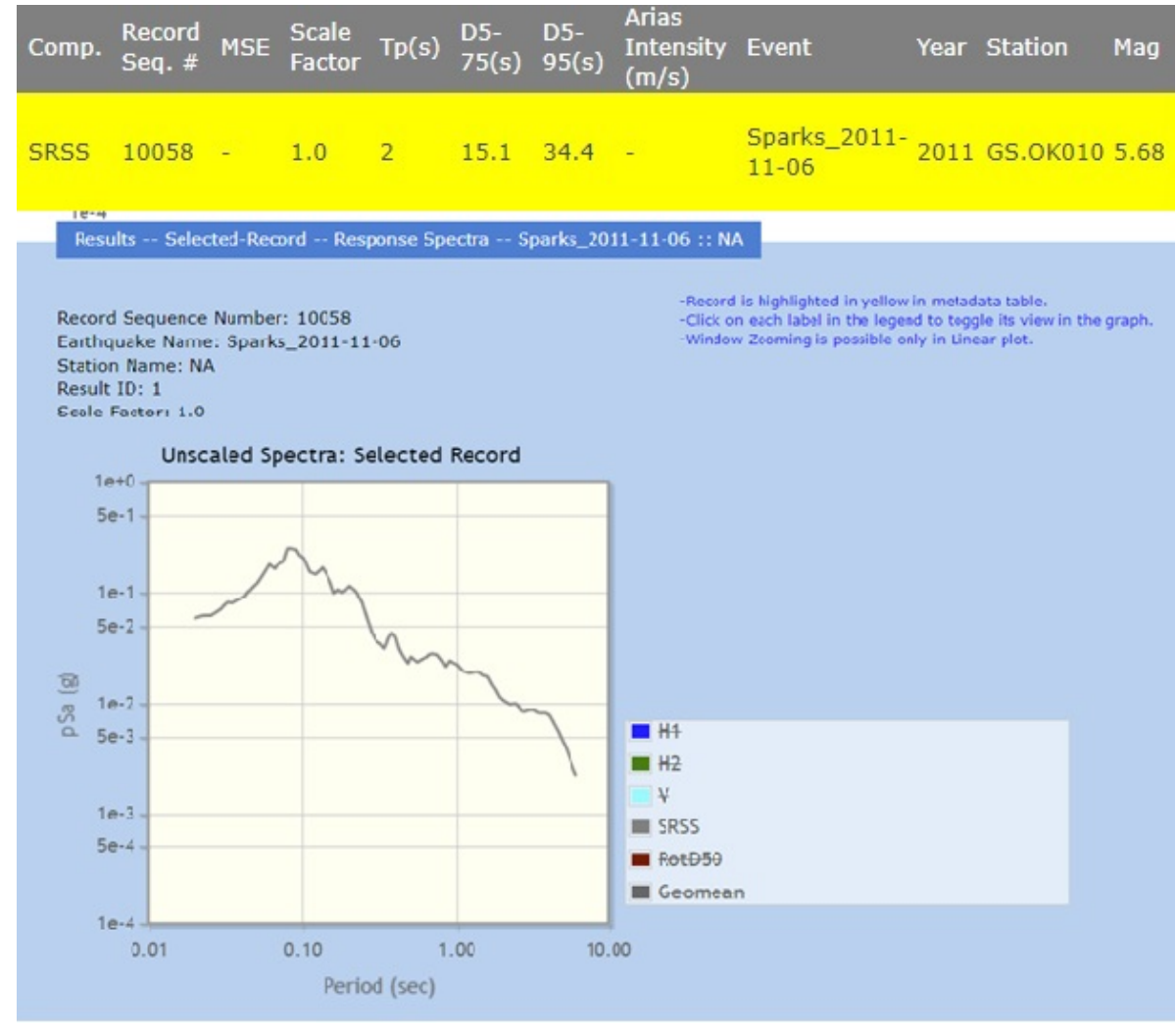

Figure 5-5: Sparks earthquake information.

To create the movement "profile" that will be the input for the SP, time series of the the horizontal component one (y axis), the horizontal component two ( $\mathrm{x}$ axis) and the vertical component( $\mathrm{z}$ axis), displayed in Figure 5-6 of the earthquake record will be used. In order to simplify the simulation, a representative section of the components mentioned before was used resulting in a 802 positions Comma Separated Values (CSV ) file that will represent the desired position of the mobile platform. 

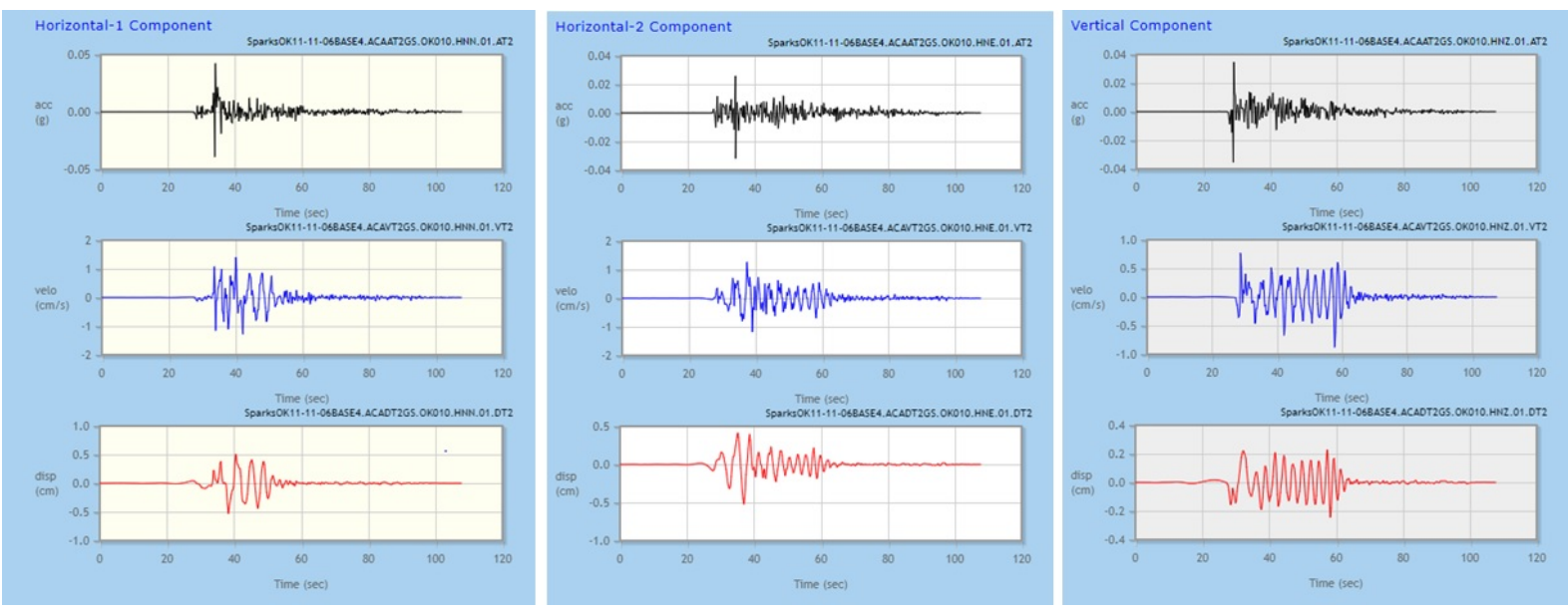

Figure 5-6: Earthquake components time series for acceleration, velocity and position.

\subsection{Linear actuator parameters}

From the datasheet of the LACT6P-12V-20" [2] it is possible to derive motor parameters as well as an equivalent linear friction parameter. The latter will consider altogether rotational friction, linear friction and the screw drive efficiency; parameters are shown in Table 5-1.

\begin{tabular}{|c|c|c|c|c|c|c|c|c|}
\hline$V_{c}[\mathrm{~V}]$ & $i_{n l}[A]$ & $i_{f l}[\mathrm{~A}]$ & $l_{n l}^{\prime}[\mathrm{mm} / \mathrm{s}]$ & $l_{f l}^{\prime}[\mathrm{mm} / \mathrm{s}]$ & lead $[\mathrm{mm} / \mathrm{rad}]$ & $g r$ & $F_{n l}[\mathrm{~N}]$ & $F_{f l}[\mathrm{~N}]$ \\
\hline 12 & 1.2 & 3.2 & 14.6 & 12.3 & $\frac{3}{2 \pi}$ & 20 & 0 & 500 \\
\hline
\end{tabular}

Table 5-1: Linear actuator parameters. Taken from [2]

\subsubsection{Electrical system of equations}

First consider the electrical equilibrium equation of a standard model for a DC motor

$$
V_{c}=i R+l \frac{d i}{d t}+K_{b} \omega_{m}
$$

Where $V_{c}$ is the input control voltage, $\mathrm{R}$ and $\mathrm{L}$ the motor resistance and inductance respectively, $K_{b}$ is the back-emf constant. For the full load velocity and no load velocity cases, the corresponding steady state values for velocities and control voltage are given; the following system of equations can be formulated, recall that $d i / d t=0$ at steady-state. 


$$
\begin{aligned}
& V_{c}=i_{n l} R+l \frac{d i}{d t}+K_{b} \omega_{m n l} \\
& V_{c}=i_{f l} R+l \frac{d i}{d t}+K_{b} \omega_{m f l}
\end{aligned}
$$

where the subscripts $f l$ and $n l$ denote the full-load and no-load case respectively. The motor angular velocity can be obtained considering that $\omega_{m}=g r * \omega_{\text {screw }}$ and $\omega_{\text {screw }}=l^{\prime} /$ lead, with lead $=3[\mathrm{~mm} / \mathrm{rev}]=\frac{3}{2 \pi}[\mathrm{mm} / \mathrm{rad}][25]$. The result of solving the system of equations is

$$
R=0.86358 \Omega ; \quad K_{b}=0.01793 \mathrm{As} / \mathrm{rad}
$$

It is missing the motor inductance $L$. That was obtained through an experimental process in which a resistance of $100 \Omega$ was connected in series to the motor. Then the frequency, $f$, of a periodic input signal is increased until the voltage across the resistance is one half of the input voltage amplitude. It means that the impedance of the remaining part of the circuit is equal to $\sqrt{3} R\left(Z_{T}=\sqrt{R^{2}+(\sqrt{3} R)^{2}}=2 R\right)$. For the linear actuator the remaining part of the circuit is an $L C$ circuit with the following impedance

$$
Z_{L C}=\frac{1}{\frac{1}{Z_{C L}}+\frac{1}{Z_{L}}}
$$

For the capacitive impedance we have $Z_{C T}=\frac{1}{j \omega C_{t}}$ while for the inductive one $Z_{L}=j \omega L$, leading to $Z_{L C}=\frac{1}{\frac{1}{j \omega L}+j \omega C_{t}}=\frac{j}{\frac{1}{\omega L}-\omega C_{t}}$, that is

$$
\begin{gathered}
\left|Z_{L C}\right|=\sqrt{3} R=\frac{1}{\frac{1}{\omega L}-\omega C_{t}} \\
\rightarrow L=\frac{1}{\frac{\omega}{\sqrt{3} R}+\omega^{2} C_{t}}
\end{gathered}
$$

We have that $C_{t}=0.15 \mu F, \omega=2 \pi(13000)$ [25], which leads to

$$
L=679.18 \mu H
$$




\subsubsection{Mechanical system of equations}

Now for the mechanical equation it is convenient to consider the torque on the lead screw transformed to linear force. The amount of work the torque produce in one turn is

$$
W=2 \pi \tau
$$

which is the same as the work produced for a corresponding linear force via the screw after one turn. Thus, with a lead* in $\mathrm{m} / \mathrm{rad}$ we have

$$
W=F 2 \pi l e a d^{*}
$$

which combined leads to

$$
F=\frac{\tau e}{\text { lead }^{*}}
$$

where the new term, $e$, is the lead screw efficiency. However we will consider all of the loses on a linear viscous friction term, i.e., $e=1$. Thus, consider the equilibrium equation

$$
F_{l s}=F_{l o a d}+\beta l^{\prime}
$$

where $F_{l s}$ is the linear force of the lead screw, $F_{\text {load }}$ is due to the load and $\beta l^{\prime}$ the loses due to friction. Using $\tau=\tau_{m}=K_{t} i$ and the gear ratio transferring such a torque to the lead screw we obtain

$$
F_{l s}=\frac{g K_{T} i}{l_{e a d}^{*}}
$$

Now we can construct a system of two equation with two unknowns as follows

$$
\begin{aligned}
& \frac{g r K_{T} i_{n l}}{\operatorname{lead}^{*}}=F_{n l}+\beta l_{n l}^{\prime} \\
& \frac{g r K_{T} i_{f l}}{\operatorname{lead}^{*}}=F_{f l}+\beta l_{f l}^{\prime}
\end{aligned}
$$


where $F_{n l}$ and $F_{f l}$ are the load force for zero load and full load. Substituting the corresponding values, see Table 5-1, and solving the system we obtain $K_{T}=0.005453[\mathrm{Nm} / \mathrm{A}]$ and $\beta=$ $18.7735[\mathrm{Ns} / \mathrm{mm}]$. The parameters are summarized in Table 5-2. The force equilibrium equation is missing the mass load, since the external load is introduce using $F_{\text {load }}$ it can be considered that the force is applied to the stroke mass, $m_{s}$. However, before the $F_{l s}$ is derived it must be considered that there is a torque load due to the rotor and screw inertia moment: that is

$$
\begin{gathered}
\tau_{\text {load }}=J_{m s} \dot{\omega}_{l s}, \quad \dot{\omega}_{l s}=l^{\prime \prime} / \text { lead } \\
J_{m s}=J_{m} g r^{2}+J_{l s} \\
J_{l s}=\frac{1}{2} m_{l s} r_{l s}^{2} \\
J_{m}=\frac{1}{2} m_{m} r_{m}^{2}
\end{gathered}
$$

where $J_{m}$ is the inertia of the rotor, notice that it is reflected to the screw side, $J_{l s}$ is the inertia of the lead screw. The rotor and screw are considered as cylinders for which their mass and radius are known, the values are summarized in Table 5-3.

\begin{tabular}{|c|c|c|c|c|}
\hline $\mathrm{R}[\Omega]$ & $\mathrm{L}[\mu F]$ & $K_{t}[\mathrm{Nm} / A]$ & $K_{b}[$ As $/ \mathrm{rad}]$ & $\beta[\mathrm{Ns} / \mathrm{mm}]$ \\
\hline 0.86358 & 679.18 & 0.005453 & 0.01793 & 18.7735 \\
\hline
\end{tabular}

Table 5-2: Linear actuator found parameters.

\begin{tabular}{|c|c|c|c|c|c|}
\hline$m_{s}[\mathrm{~kg}]$ & $m_{m}[\mathrm{~kg}]$ & $m_{l s}[\mathrm{~kg}]$ & $r_{l s}[\mathrm{~m}]$ & $r_{m}[\mathrm{~m}]$ & $J_{m s}\left[\mathrm{kgm}^{2}\right]$ \\
\hline $73 \times 10^{-3}$ & $130 \times 10^{-3}$ & $118.345 \times 10^{-3}$ & $5.2 \times 10^{-3}$ & $12.5 \times 10^{-3}$ & $4.0641 \times 10^{-3}$ \\
\hline
\end{tabular}

Table 5-3: Rotor, screw parameters and combined inertia.

\subsubsection{Motor model}

The linear actuator assembled in the HxCf1 consist on a direct current motor that moves a lead screw in order to get to the desired position. After analysing the electrical and mechanical characteristics and finding the parameters of the actuator, a Simscape model of the DC motor 
is proposed. This motor model have a control voltage of $12 \mathrm{~V}$ as input and linear velocity and position of the actuator as output (see Figure 5-7).

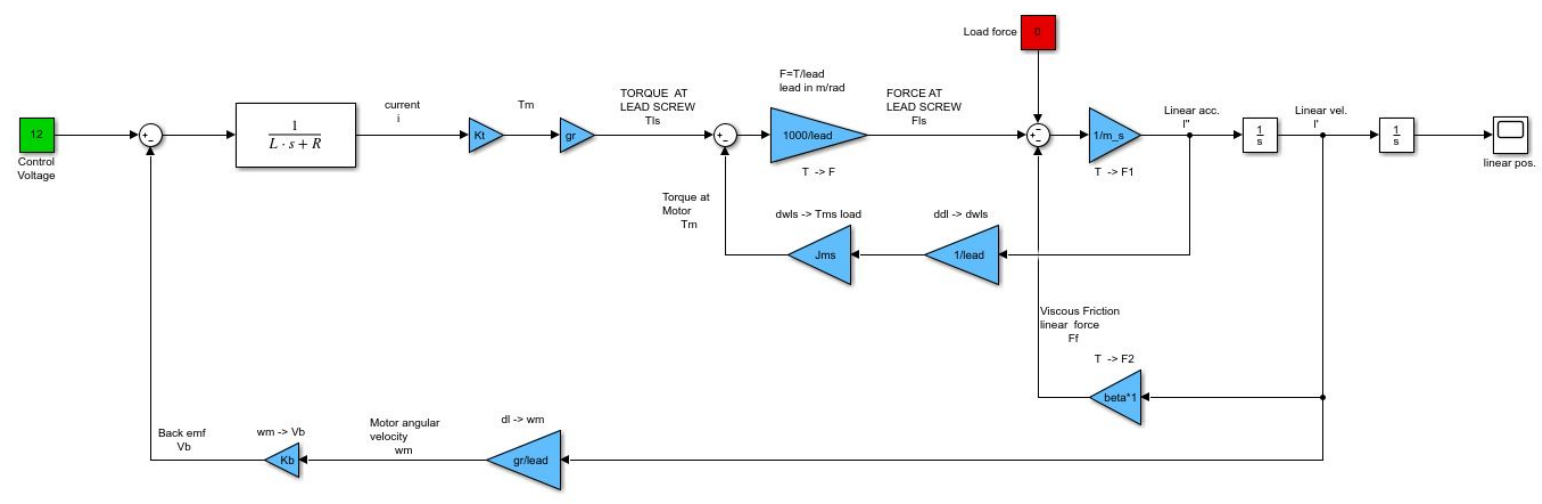

Figure 5-7: Simscape DC motor model.

In order to test the Direct current (DC ) motor model, the two equilibrium conditions were tested, the first one with no external load $(0 \mathrm{~N})$, and the second one with full load capacity (500 N); after performing the simulation, the linear velocity was plotted in order to see if the maximum velocity in each condition corresponds to the one defined in the actuator data sheet [2] by the fabricator. The resulting plots shown in Figure 5-8 show that the velocity in each condition corresponds to the given ones by the fabricator (see Figure 5-9).

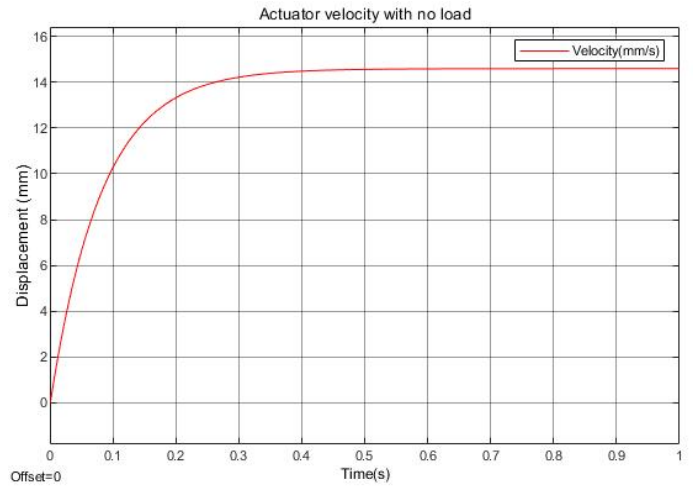

(a) Response with no load.

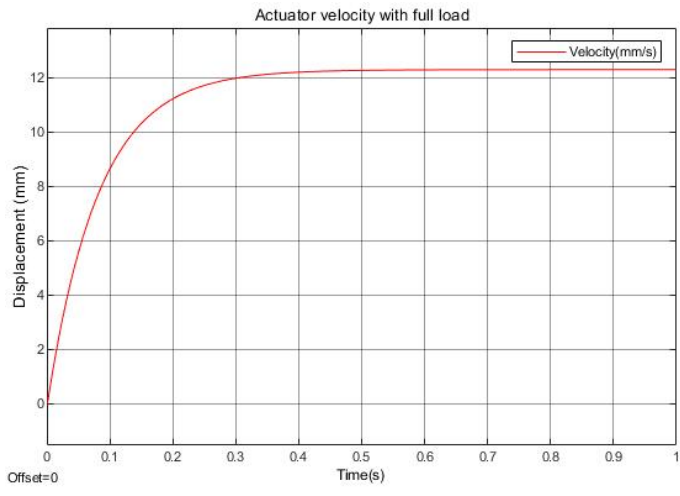

(b) Response with full load.

Figure 5-8: Simscape DC motor model simulation. 


\begin{tabular}{|c|c|c|c|c|c|c|}
\hline $\begin{array}{c}\text { Gear } \\
\text { Ratio }\end{array}$ & \multicolumn{2}{|c|}{ Max Load/Dynamic } & \multicolumn{2}{c|}{ Speed/No Load } & \multicolumn{2}{c|}{ Speed/Max Load } \\
\hline & Lbs. [US] & Newtons & inches/sec & $\mathrm{mm} / \mathrm{sec}$ & inches/sec & $\mathrm{mm} / \mathrm{sec}$ \\
\hline $5: 1$ & 34 & 150 & 1.73 & 43.9 & 1.44 & 36.5 \\
\hline $10: 1$ & 56 & 250 & 1.09 & 27.6 & 0.93 & 23.5 \\
\hline \hline $20: 1$ & 112 & 500 & 0.57 & 14.6 & 0.48 & 12.3 \\
\hline \hline $30: 1$ & 180 & 800 & 0.37 & 9.5 & 0.30 & 7.5 \\
\hline $40: 1$ & 225 & 1000 & 0.28 & 7.0 & 0.22 & 5.5 \\
\hline
\end{tabular}

Figure 5-9: Linear actuator performance data. Taken from [2]. 


\section{PID Controller}

Since Ziegler and Nichols Proportional Integral Derivative (PID ) empirical tuning rules had been published in 1942 [41], the PID control has survived the challenge of advanced control theories. The PID's long life force comes from its clear meaning and effectiveness in practice. In PID control, the P-control (Proportional control) is the present effort making a present state into desired state, I-control (Integral control) is the accumulated effort using the experience information of bygone state and D-control (Derivative control) is the predictive effort reflecting the tendency information for ongoing state.

PID control has been utilized irrespective of linear system or nonlinear one, electrical system or mechanical one, time-invariant system or time-varying one. The strongest advantage of PID control is a 'simplicity' itself; the simple control is preferable to the complex control at least in industry, if the performance enhanced by using complex control does not stand in different levels.

It is important to highlight that the trajectory tracking control system is quite different from the regulation control systems in that the desired configurations which are functions of time are inserted in equation of motion for mechanical system. Hence, the trajectory tracking system has the time-varying characteristics because of desired configurations [15].

In this chapter, the Pololu jrk default motor controller is going to be tested in order to observe its performance and a new proposal of control is going to be developed, tested and compared with the default one, in order to observe the differences in the response and the performance in trajectory tracking. 


\subsection{Pololu Jrk default motor controller}

The CIDESI hexapod HxCf1 uses the pololu LACT6P generic actuators as legs, and following the fabricator recommendations, the motor controller used is the Jrk 21v3. The Jrk 21v3 is a versatile, general-purpose motor controller that supports a variety of interfaces, including USB. The broad operating range from $5 \mathrm{~V}$ to $28 \mathrm{~V}$ and continuous output current of $3 \mathrm{~A}$ (5 A peak) allow this board to control most small DC brushed motors. Analog voltage and tachometer (frequency) feedback options allow quick implementation of closed-loop servo systems, and a free configuration utility (for Windows) allows easy calibration and configuration through the USB port [9].

Configurable parameters of the motor controller include:

- PID period and PID constants (feedback tuning parameters).

- Maximum current.

- Maximum duty cycle.

- Maximum acceleration.

- Error response.

- Input calibration (learning) for analog and RC control.

The fabricator offers in their web page, a generic configuration for the motor controller. For the master thesis purposes, we are going to highlight the PID values; the default configuration is a PD control with a value for the proportional gain of $K_{p}=35$, the value for the integral gain of $K_{i}=0$ and the value for the derivative gain of $K_{D}=60$.

In order to test the performance of one actuator with the default configuration, a simulation was performed using the model shown in Figure 6-1; in the simulation, the input was the earthquake profile in actuator space coordinates and the output was the error while performing the routine, having an external load of $0 N$. The error graph, shown in Figure 6-2, display the performance of this configuration, having a mean squared error of $M S E=0.02491$. The Bode plots generated in the simulation (see Figure 6-3 and Figure 6-4), shows the frequency response of the system. 


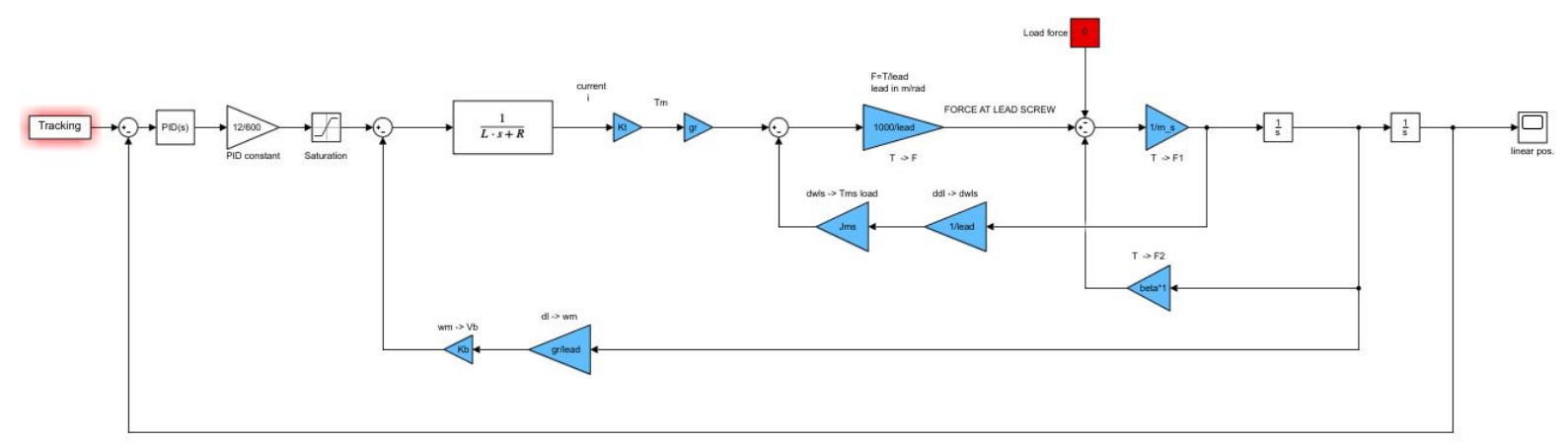

Figure 6-1: Closed loop DC motor model.

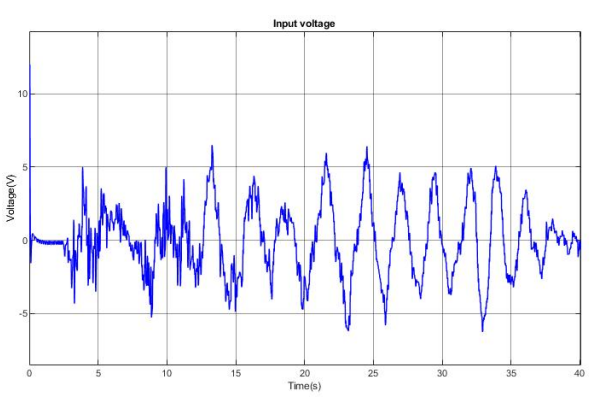

(a) PD Control voltage.

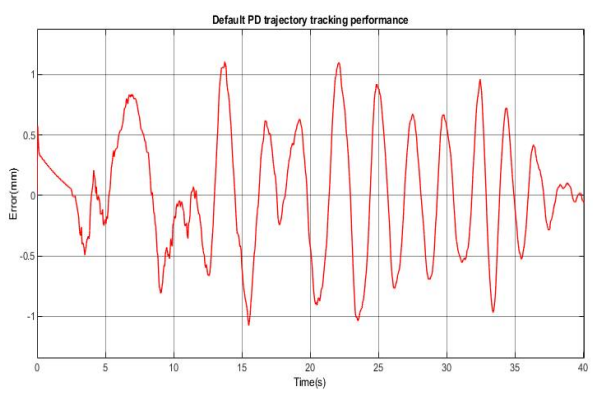

(b) PD Trajectory Tracking error.

Figure 6-2: Default PD trajectory tracking performance.

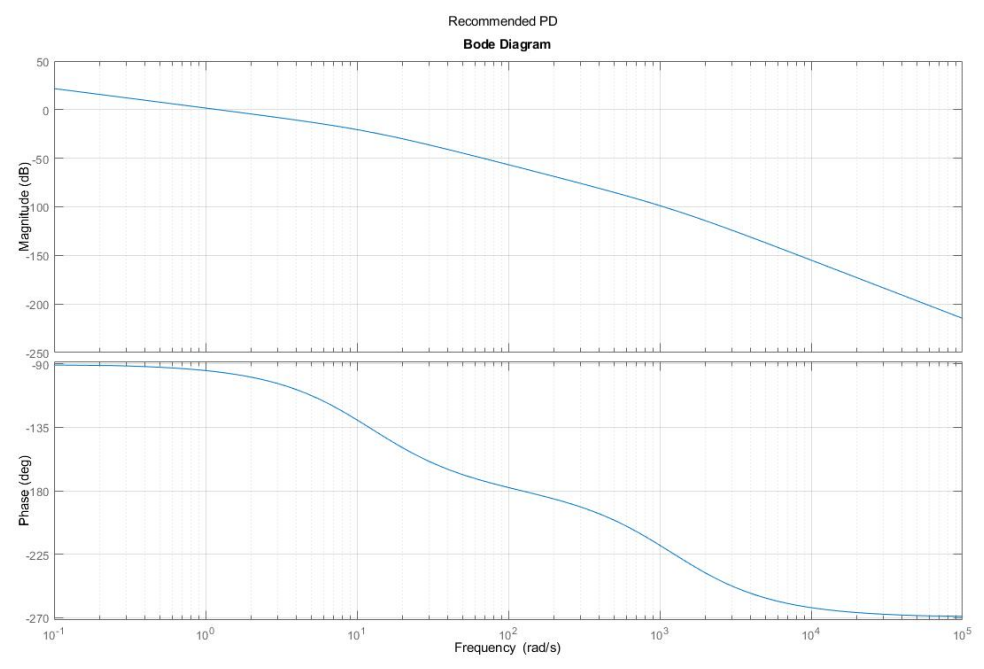

Figure 6-3: Default PD open loop Bode plot. 


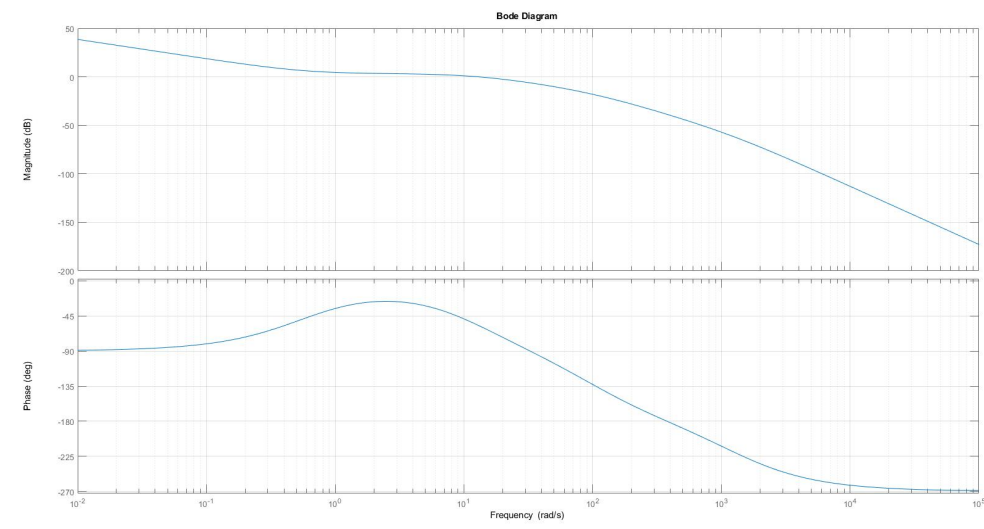

Figure 6-4: Plant Bode plot.

\subsection{Ziegler Nichols tuning}

The Ziegler-Nichols method allows to adjust or "tune" a PID controller empirically, without knowing the plant or the controlled system equations. These adjustment rules proposed by Ziegler and Nichols [41] were published in 1942 and since then it is one of the most widely used and widely used tuning methods. The values proposed by this method try to achieve in the feedback system a step response with a maximum overshoot of $25 \%$, which is a robust value with good characteristics of speed and stability for most systems.

The Ziegler-Nichols PID tuning method allows proportional, integral and derivative gains to be defined from the response of the open loop system or from the response of the closed loop system; each test fit better to different type of systems. For the proposed DC motor model, the closed loop method is the one that should be used.

The test consist of setting the derivative action and the integral action of the PID controller to zero and gradually increase the proportional gain until the system oscillates in a sustained manner; this oscillation must be linear, without saturation. At this time the proportional gain, called critical gain or $K_{c}$, and the oscillation period $T_{c}$ in seconds must be measured.

Once these two values are measured, the PID controller parameters can be calculated with proportional and integral (PI), proportional and derivative (PD) or proportional integral and derivative (PID) using Table 6-1 where the constant $K_{p}$ corresponds to the proportional gain, $K_{I}$ is the integral gain and $K_{D}$ is the derivative gain.

This tuning method was tested in the system but the gain that produces sustained oscillations 


\begin{tabular}{|c|c|c|c|}
\hline Control & $K_{p}$ & $K_{i}$ & $K_{D}$ \\
\hline $\mathrm{P}$ & $0.5 * K_{c}$ & & \\
\hline $\mathrm{PI}$ & $0.45 * K_{c}$ & $0.54 * K_{c} / T_{c}$ & \\
\hline $\mathrm{PD}$ & $0.8 * K_{c}$ & & $0.75 * K_{c} * T_{c}$ \\
\hline $\mathrm{PID}$ & $0.59 * K_{c}$ & $1.18 * K_{c} / T_{c}$ & $0.75 * K_{c} * T_{c}$ \\
\hline
\end{tabular}

Table 6-1: Tuning formulas for $K_{p}, K_{I}$ and $K_{D}$.

leads to a very big proportional gain. In addition, a very big power supply would be necessary, and that is impossible for this system; after testing and identifying this characteristics, this tuning method was discarded and other one was proposed in order to improve the performance of the system.

\subsection{PID proposal}

The main goal of the PID controller is to improve the performance of the system, and the default configuration is normally not the optimal in order to obtain the desired results. Based on the bode plots of the proposed DC motor model, because Equation 6.1 is the PID controller in order to define the values for $K_{p}, K_{I}$ and $K_{D}$. Using this gains will allow the system to improve disturbance rejection, add phase lead, improve phase margin,and speeding up the response.

$$
C(s)=\frac{K_{p} s+K_{D} s^{2}+K_{I}}{s}
$$

Using a gain of $K=70$ and suitable values for zeros $a=1$ and $b=10$, ans substituting the values in Equation 6.2 we obtain

$$
\begin{gathered}
C(s)=\frac{K}{s}(s+a)(s+b) \\
C(s)=\frac{70 s^{2}+770 s+700}{s}
\end{gathered}
$$

From Equation 6.3 and taking as base Equation 6.1 we can obtain the proportional gain $K_{p}=$ 770, the integral gain $K_{I}=700$ and the derivative gain $K_{D}=70$. With the obtained values, the configurable PID of the motor model was changed and the same simulation was performed with the same conditions used with the default PD controller speeding up the response and improving 
the phase margin. In Figure 6-5 the trajectory tracking performance of the controller (control voltage and position error) is shown, having a mean square error of $\mathrm{MSE}=0.0180$, improving the previous performance with the default PD; also we can observe in Figure 6-6 the bode plot of this configuration, having the crossover frequency in $15 \mathrm{rad} / \mathrm{s}$, pretty close to the calculated frequency in the system characterization.

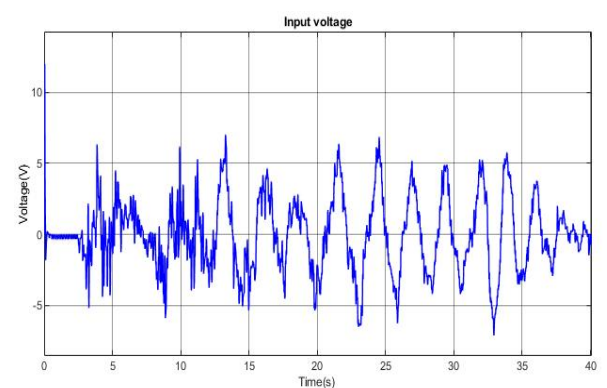

(a) PID Control voltage.

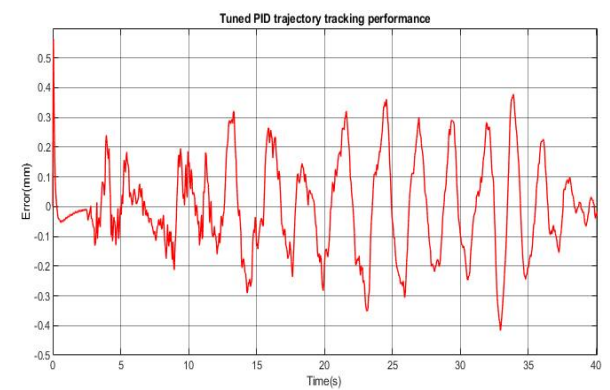

(b) PID Trajectory Tracking error.

Figure 6-5: Tuned PID trajectory tracking performance.

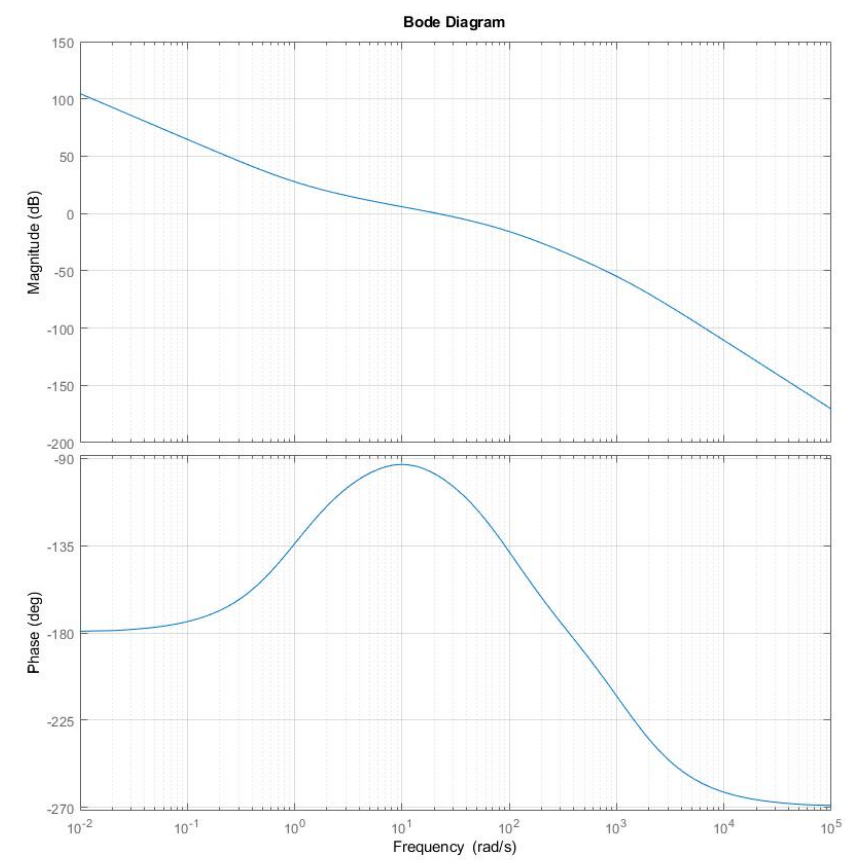

Figure 6-6: Tuned PID Bode plot. 


\section{Earthquake simulations}

This chapter presents the earthquake ground motion simulation of the SP dynamic model using the default PD controller and the proposed PID controller with their corresponding results and plots.

\subsection{Default PD simulation}

In order to perform a simulation, an input file containing the required positions and orientations for the Stewart Platform is used; for this simulation a real earthquake ground motion obtained from PEER database [11] was used as input. This specific earthquake was chosen because the length of the movements were in the order of millimetres and it was recorder close to México, that is the target region for this work.

In the simulation, a representative part of the earthquake was taken and the reference positions are updated at a $50 \mathrm{~ms}$ rate, having a total length of the simulation of 40 seconds; the default PD configuration was used and after running the simulation, the leg length values obtained by the hexapod model output are compared with the desired leg length values calculated by the inverse kinematics and the leg length errors of the actuators 1 to 6 are calculated. The resulting plots are shown in Figure 7-1 having the leg length error plots shown in Figure 7-2.

For the mobile platform position in the $\mathrm{x}, \mathrm{y}, \mathrm{z}$ axis, the position sensor mounted on the top of the mobile platform data was compared against the desired platform position data; desired against actual position plots are shown in Figure 7-3 and the error plots are shown in Figure 7-4. The control voltage plots for Actuator 1 to 6 are shown in Figure 7-5. The MSE for each actuator and for the mobile platform coordinate system are summarized in Table 7-1. 


\begin{tabular}{|c|c|c|c|c|c|c|c|c|c|}
\hline & Leg 1 & Leg 2 & Leg 3 & Leg 4 & Leg 5 & Leg 6 & x Axis & y Axis & z Axis \\
\hline MSE & 0.2491 & 0.2252 & 0.1989 & 0.2277 & 0.2053 & 0.2003 & 0.2932 & 0.3390 & 0.1980 \\
\hline
\end{tabular}

Table 7-1: PD MSE of actuators and mobile platform.
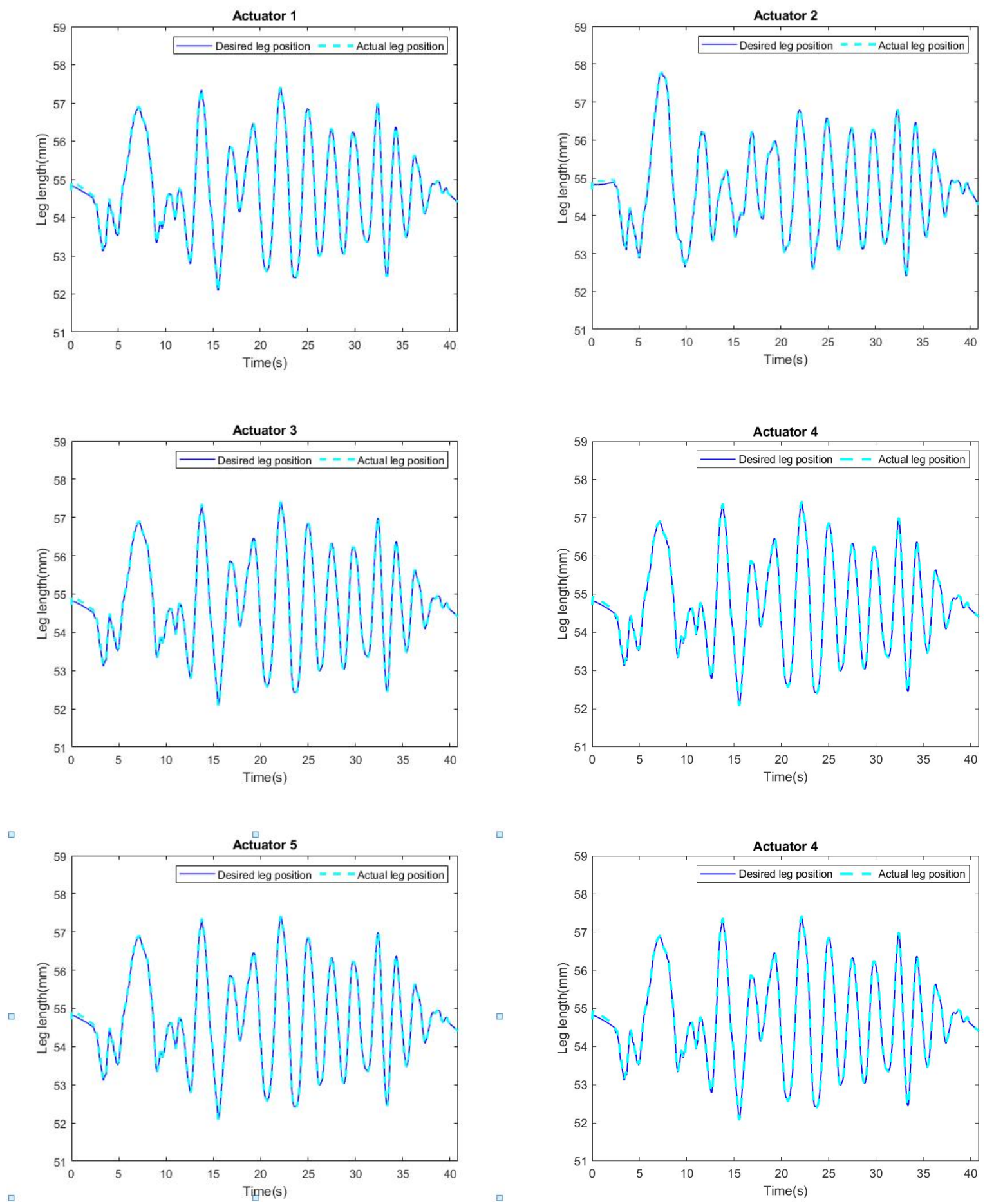

Figure 7-1: PD desired against real actuator Length. 

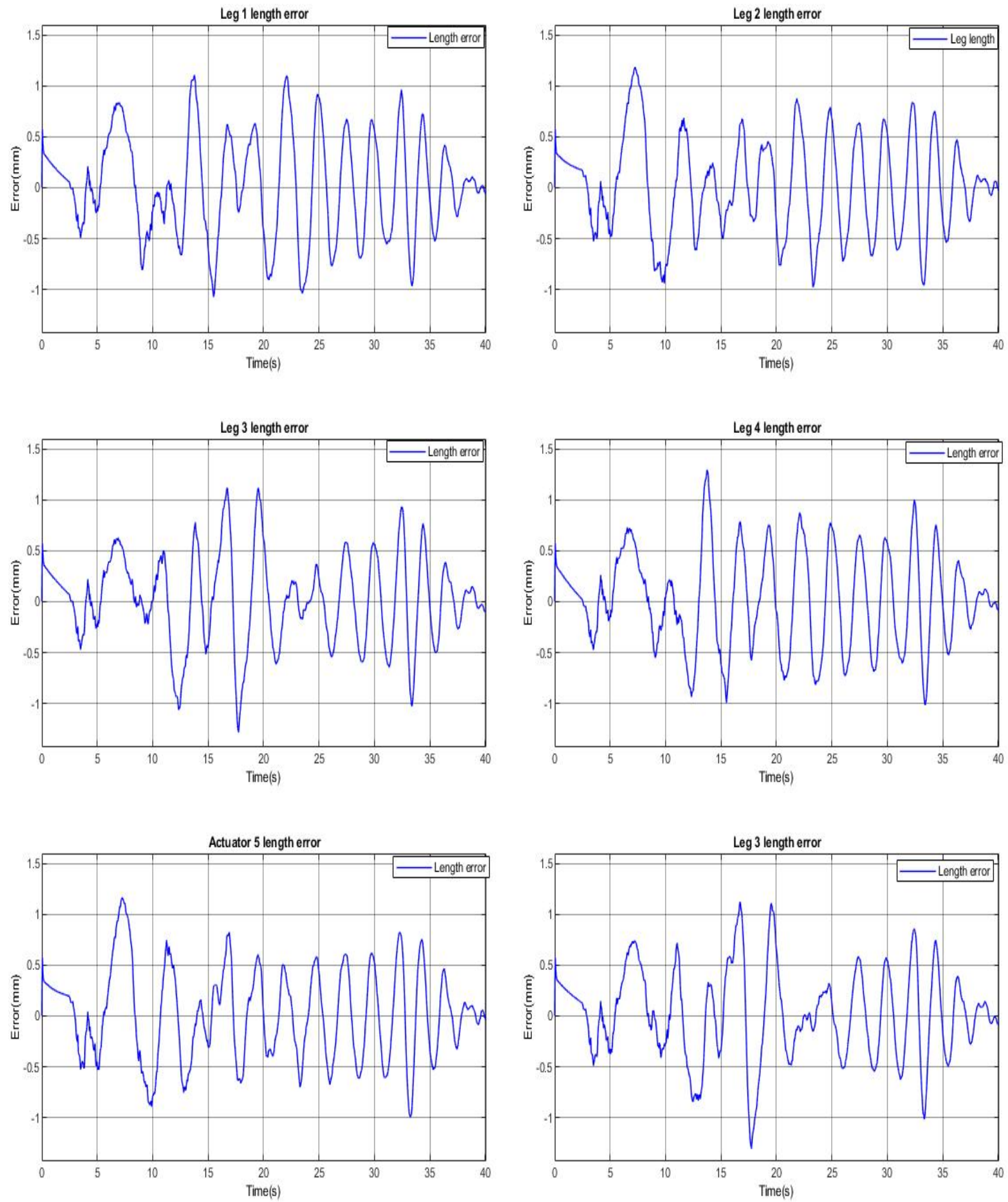

Figure 7-2: PD leg length error. 

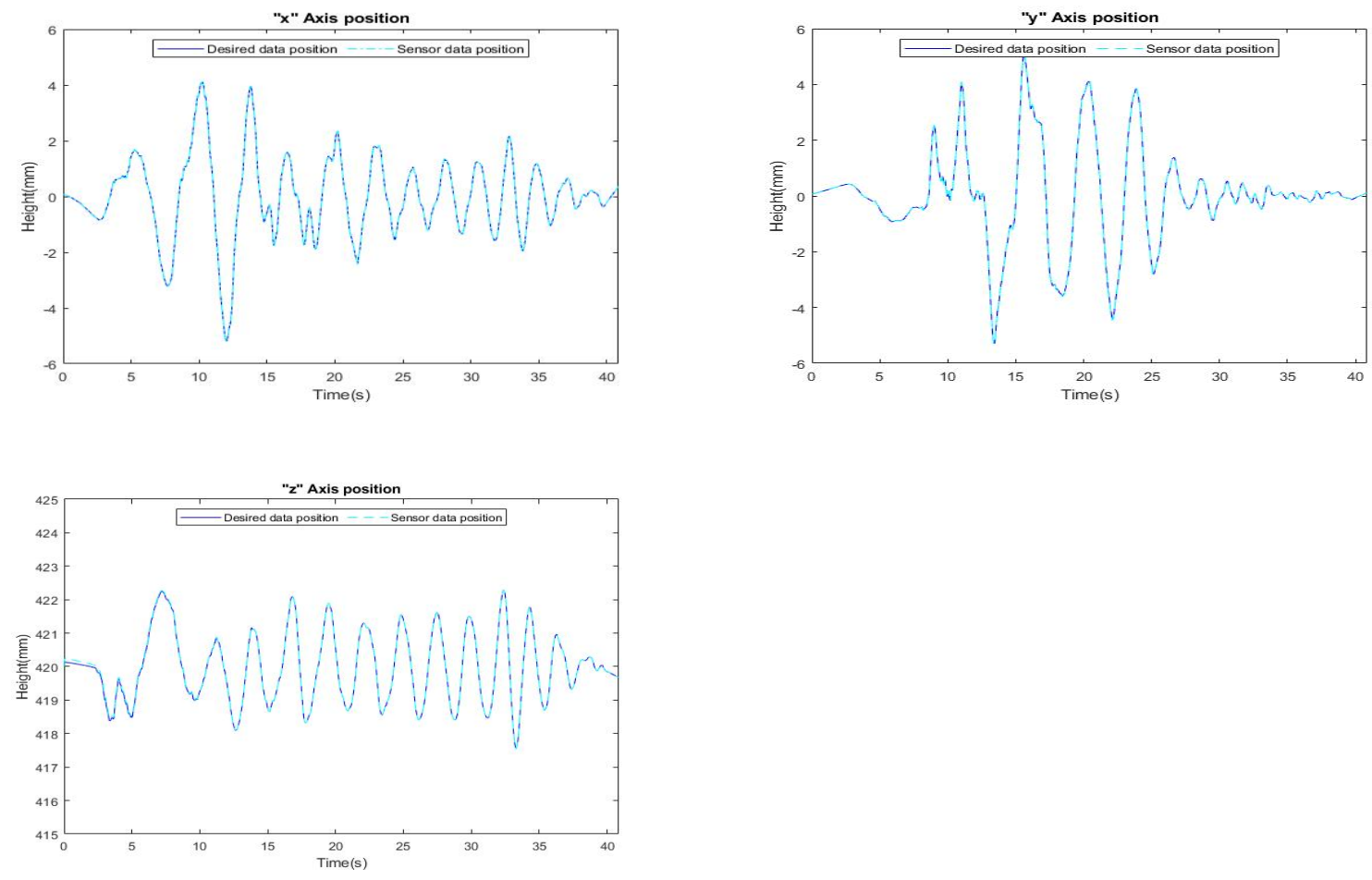

Figure 7-3: PD mobile platform axis position.
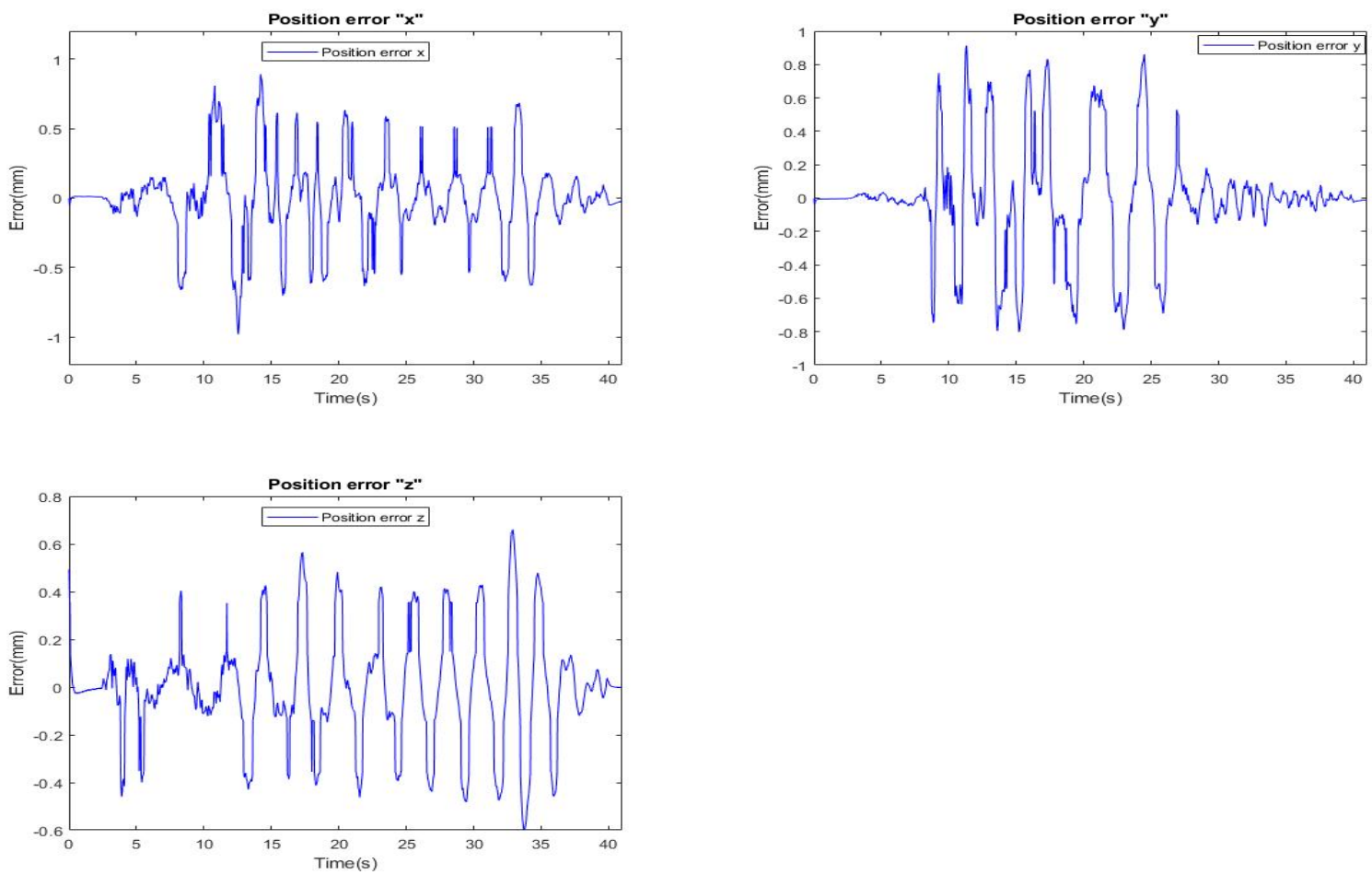

Figure 7-4: Default PD mobile platform axis error. 

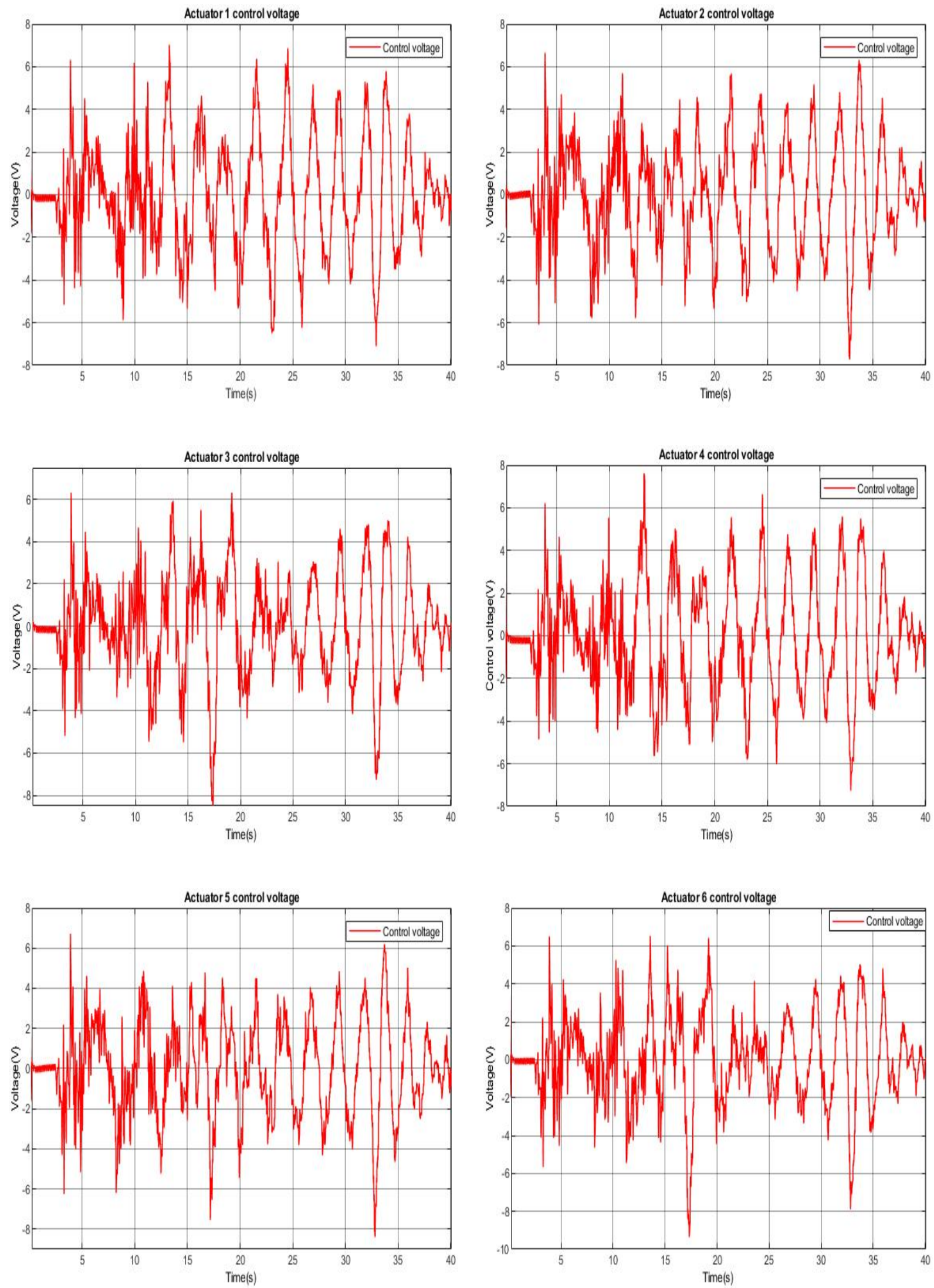

Figure 7-5: PID control voltage for each actuator. 


\subsection{Proposed PID simulation}

The second simulation was done using the PID controller configuration proposed in the previous chapter, with $K_{p}=770, K_{I}=700$ and $K_{D}=70$. After running the simulation, the leg length values obtained by the hexapod model output are compared with the desired leg length values calculated by the inverse kinematics and the leg length errors of the actuators 1 to 6 are calculated. The resulting plots are shown in Figure 7-6 having the leg length error plots shown in Figure 7-7.

For the mobile platform position in the $\mathrm{x}, \mathrm{y}, \mathrm{z}$ axis, the position sensor mounted on the top of the mobile platform data was compared against the desired platform position data; desired against actual position plots are shown in Figure 7-8 and the error plots are shown in Figure 7-9. The control voltage plots for Actuator 1 to 6 are shown in Figure 7-10 and the MSE for each actuator and for the mobile platform coordinate system are summarized in Table 7-2.

\begin{tabular}{|c|c|c|c|c|c|c|c|c|c|}
\hline & Leg 1 & Leg 2 & Leg 3 & Leg 4 & Leg 5 & Leg 6 & x Axis & y Axis & z Axis \\
\hline MSE & 0.0180 & 0.0159 & 0.0154 & 0.0177 & 0.0148 & 0.0146 & 0.0227 & 0.0249 & 0.0150 \\
\hline
\end{tabular}

Table 7-2: PID MSE of actuators and mobile platform. 

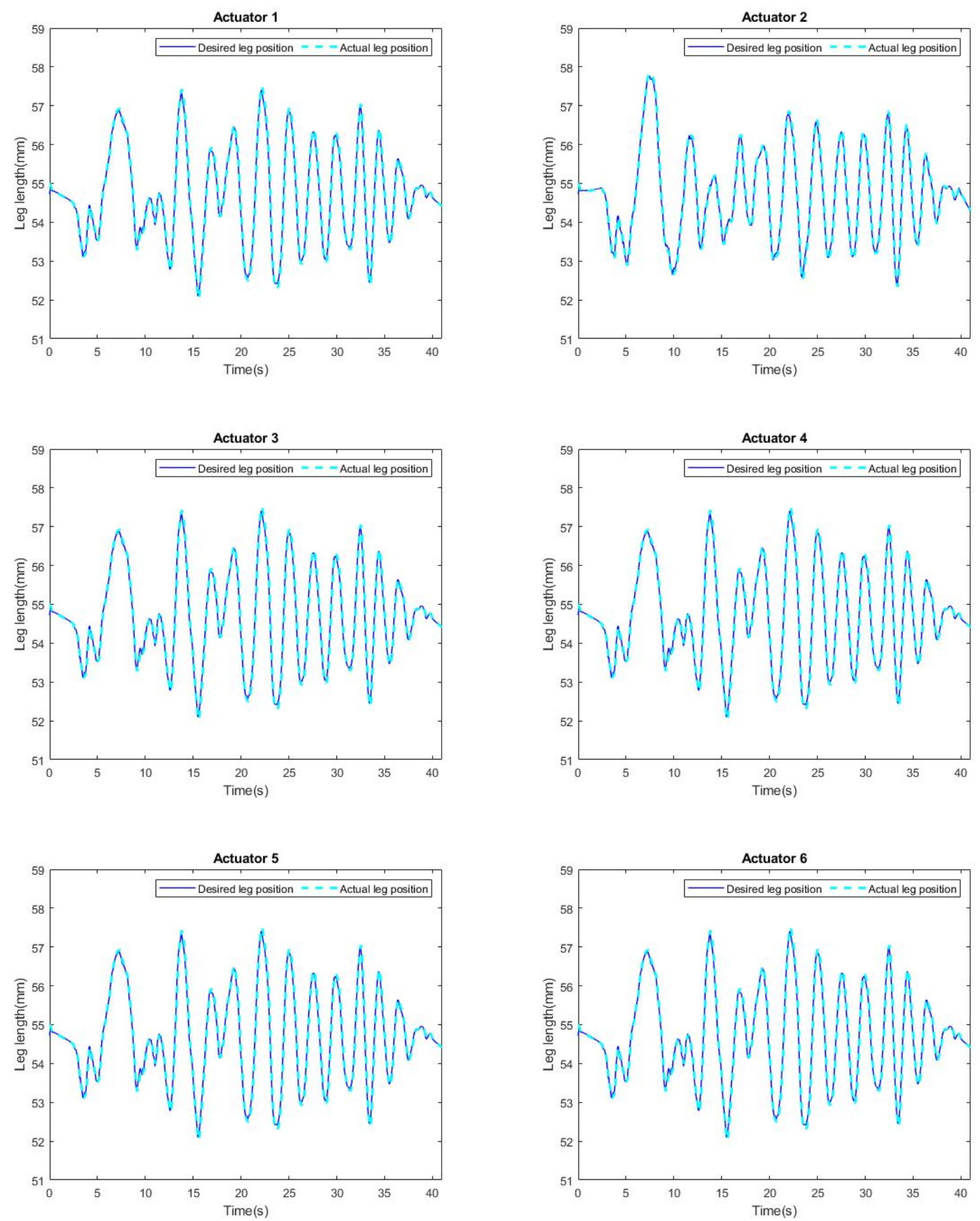

Figure 7-6: PID desired against real actuator Length. 

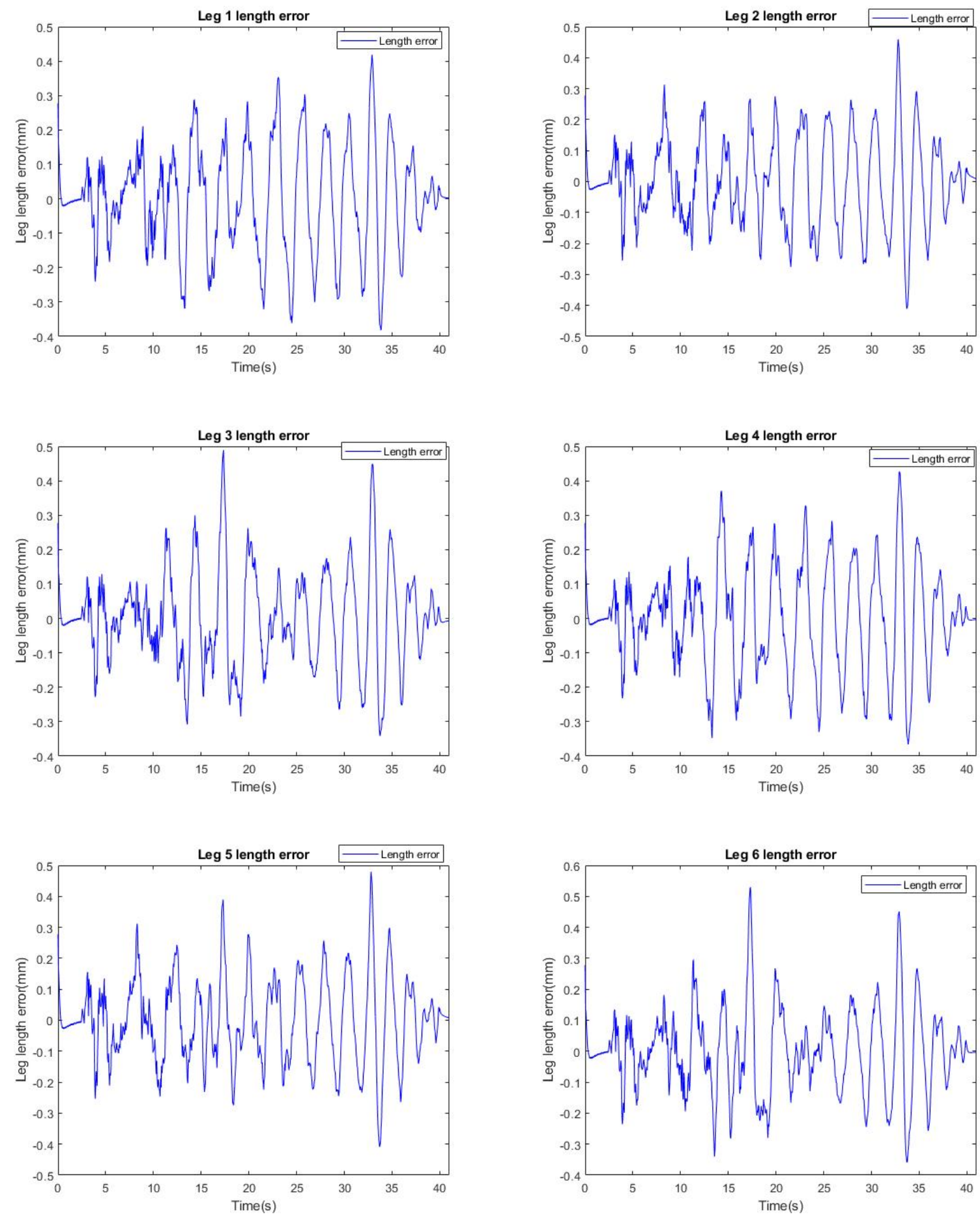

Figure 7-7: PID leg length error. 

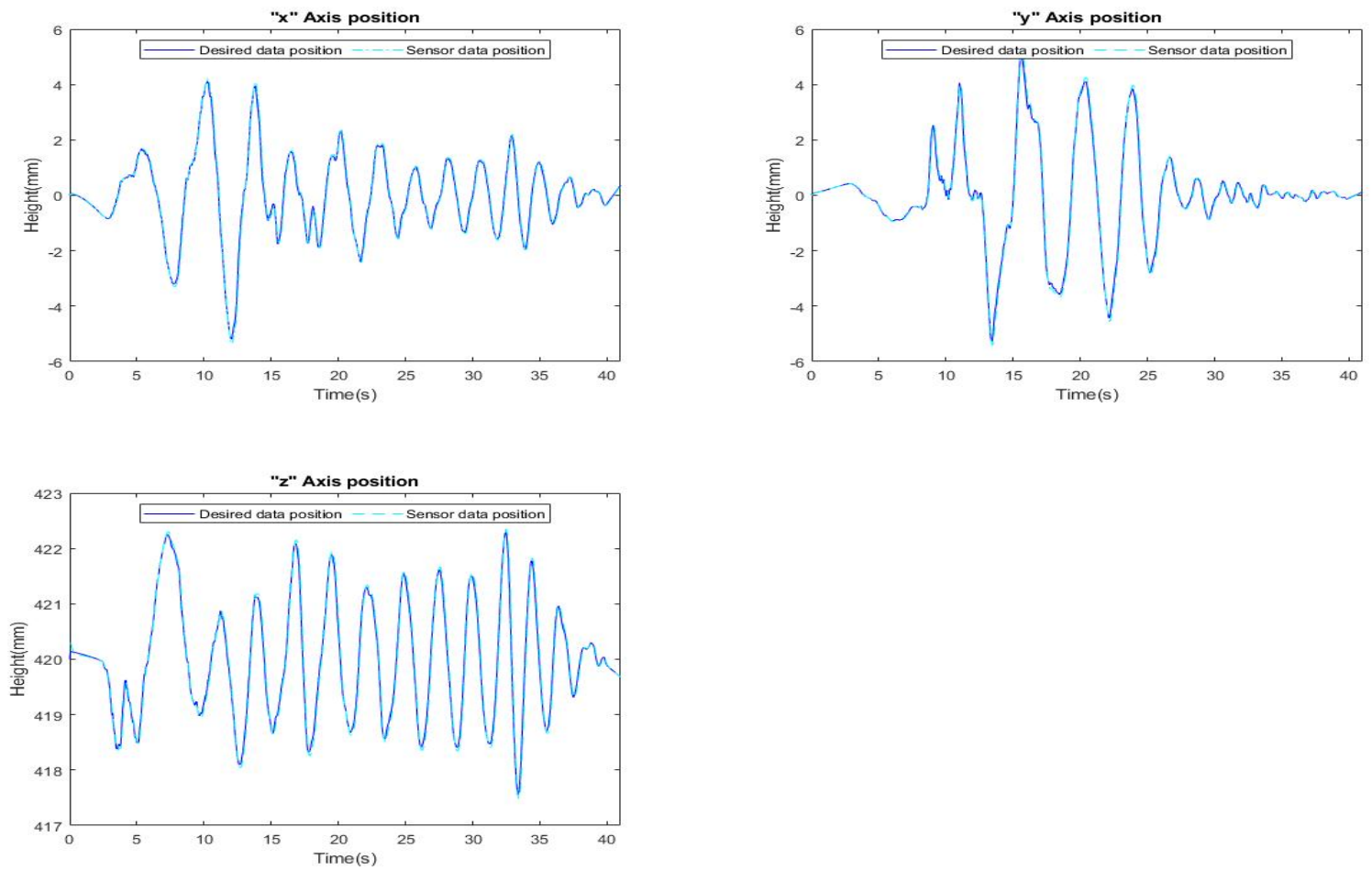

Figure 7-8: PID mobile platform axis position.
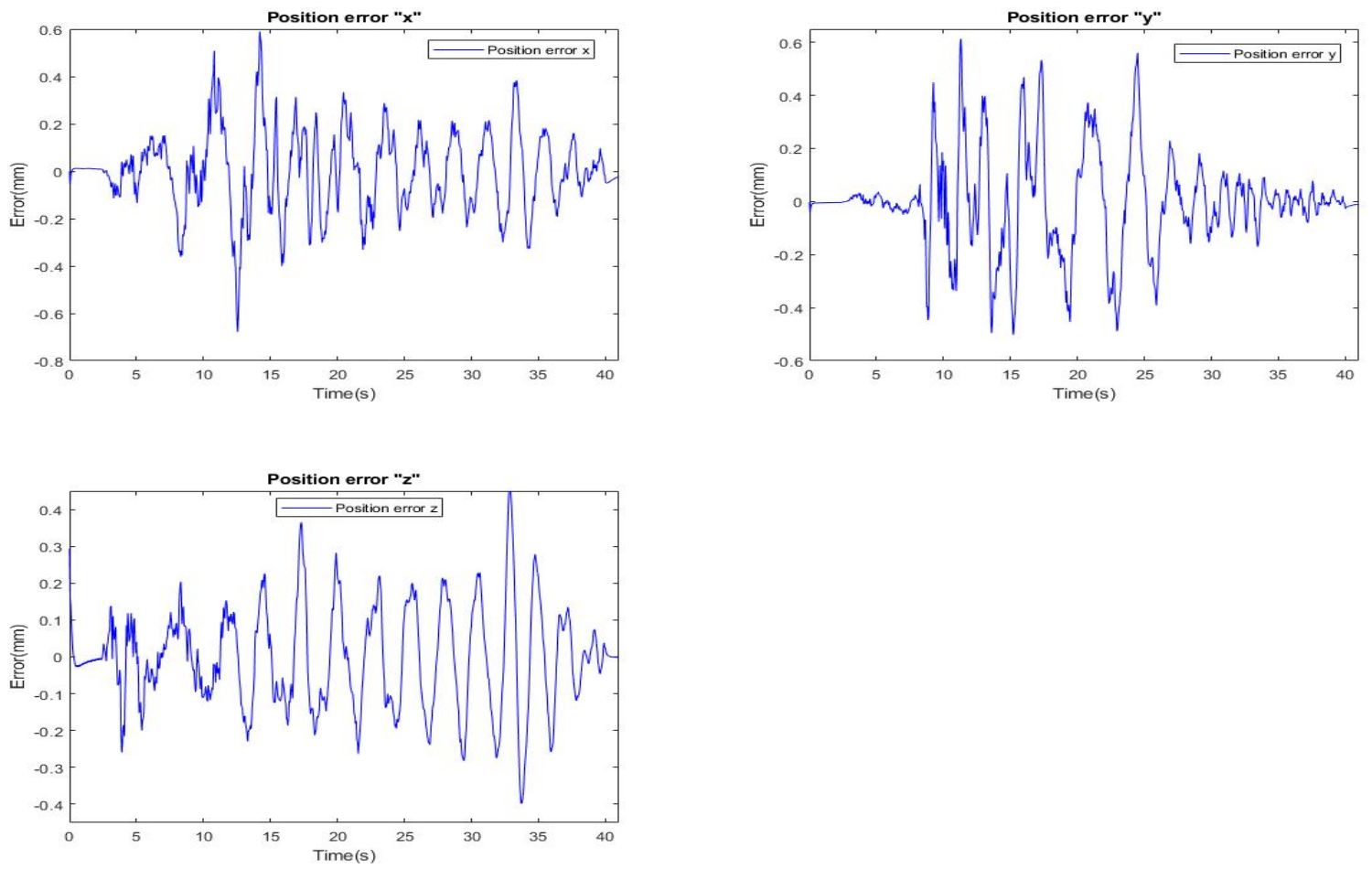

Figure 7-9: PID mobile platform axis error. 

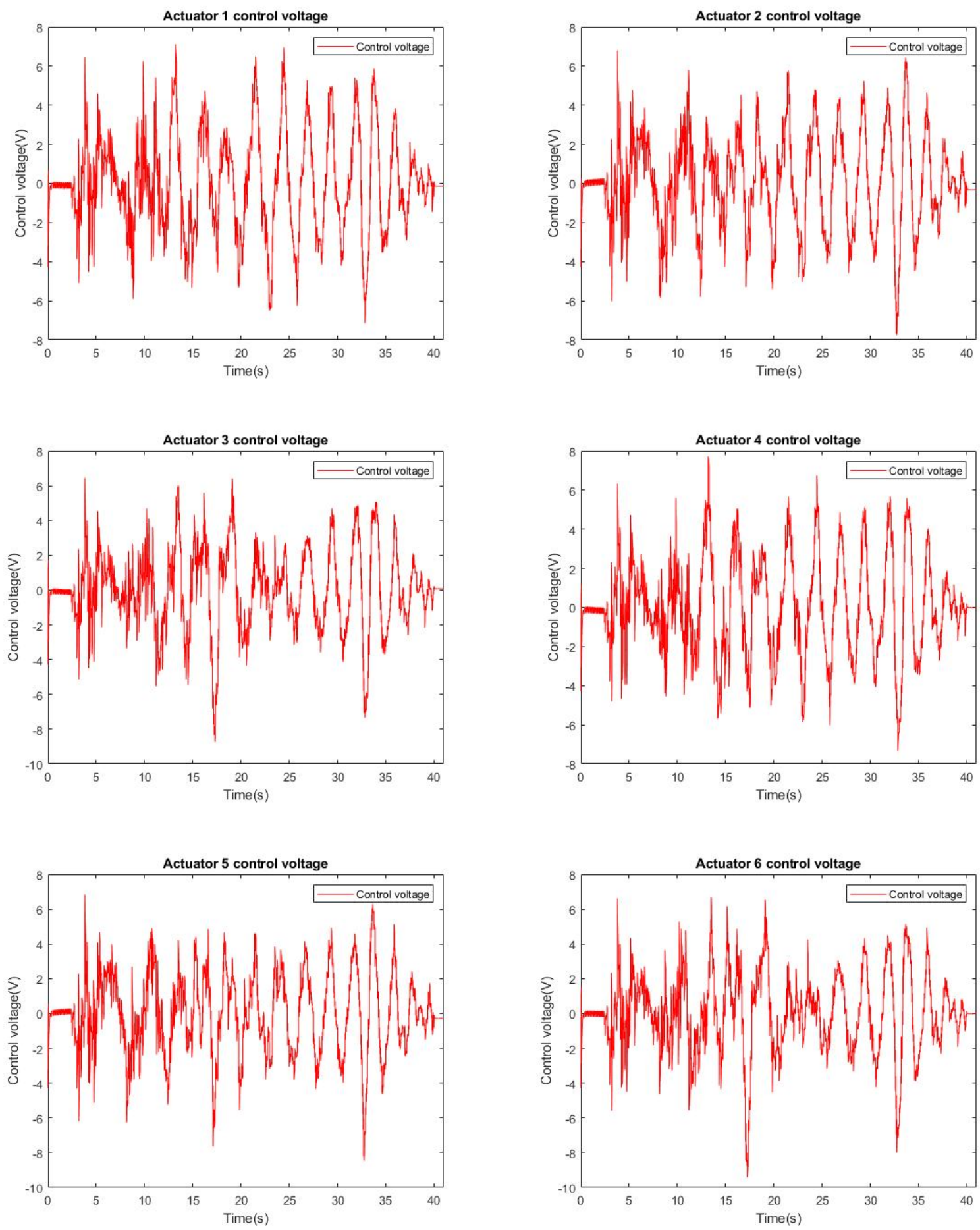

Figure 7-10: PID control voltage for each actuator. 


\section{Experimentation}

This chapter presents the experimental tests of the CIDESI HxCf1 Hexapod using an earthquake ground motion profile with the default PD controller and the proposed controller with the corresponding result plots.

\subsection{Default PD experimental results}

In order to perform experimental tests and be able to compare the results with the results obtained with the Simulink dynamic model, an Arduino program using FreeRTOS library was written; the program allows to read the position of the actuators, using their own potentiometer, at a 50 ms rate.

After running the experiment with the default PD controller and with the measurements done, the real position of the actuators can be compared with the desired position that was used as input to the SP. The resulting plots shown in Figure 8-1 display the desired against the measured leg length for the actuators 1, 3 and 5. The plots shown in Figure 8-2 show the position error also for actuators 1,3 and 5 .

In order to measure the control voltage a powerDNA cube and Labview software were used. When the outputs for the motor from the driver were connected to the cube, the routine was performed and the reading were taken. PWM signal was obtained, so it was necessary to use an RC filter in order to obtain the corresponding voltages. The control voltage plot is shown in Figure 8-3.

The measured positions using the actuator potentiometers is very noisy for each measurement; it was necessary to use a filter in order to obtain a more realistic result. After passing the data trough a first order filter with a time constant of $1 \mathrm{sec}$, the corresponding plots are shown in Fig. 8-4. The MSE of each actuator is summarized in Table 8-1. 


\begin{tabular}{|c|c|c|c|}
\hline & Leg 1 & Leg 3 & Leg 5 \\
\hline MSE & 1.1751 & 2.2415 & 0.4482 \\
\hline
\end{tabular}

Table 8-1: PD MSE of actuators 1, 3 and 5.
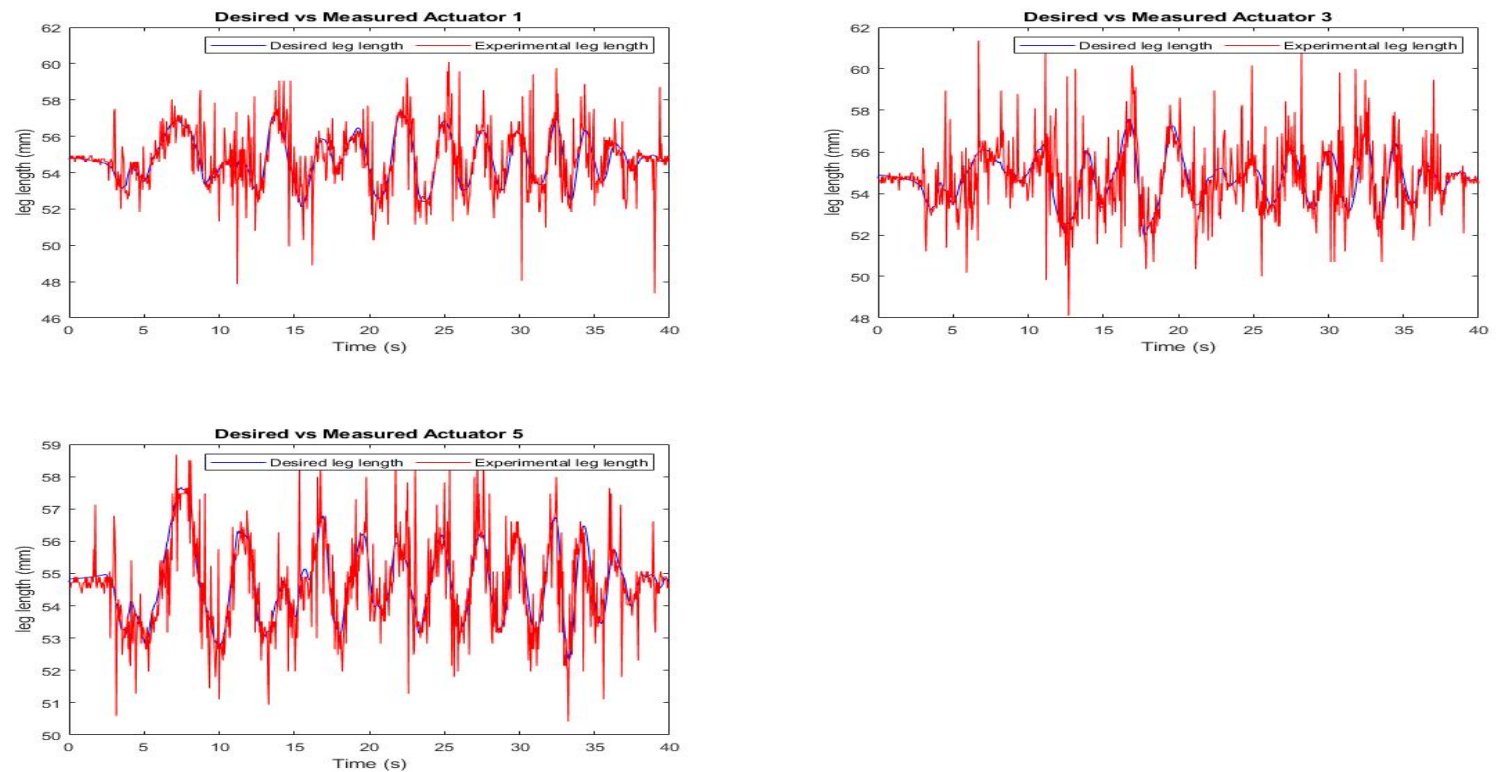

Figure 8-1: Default PD actuator measured positions against desired positions.
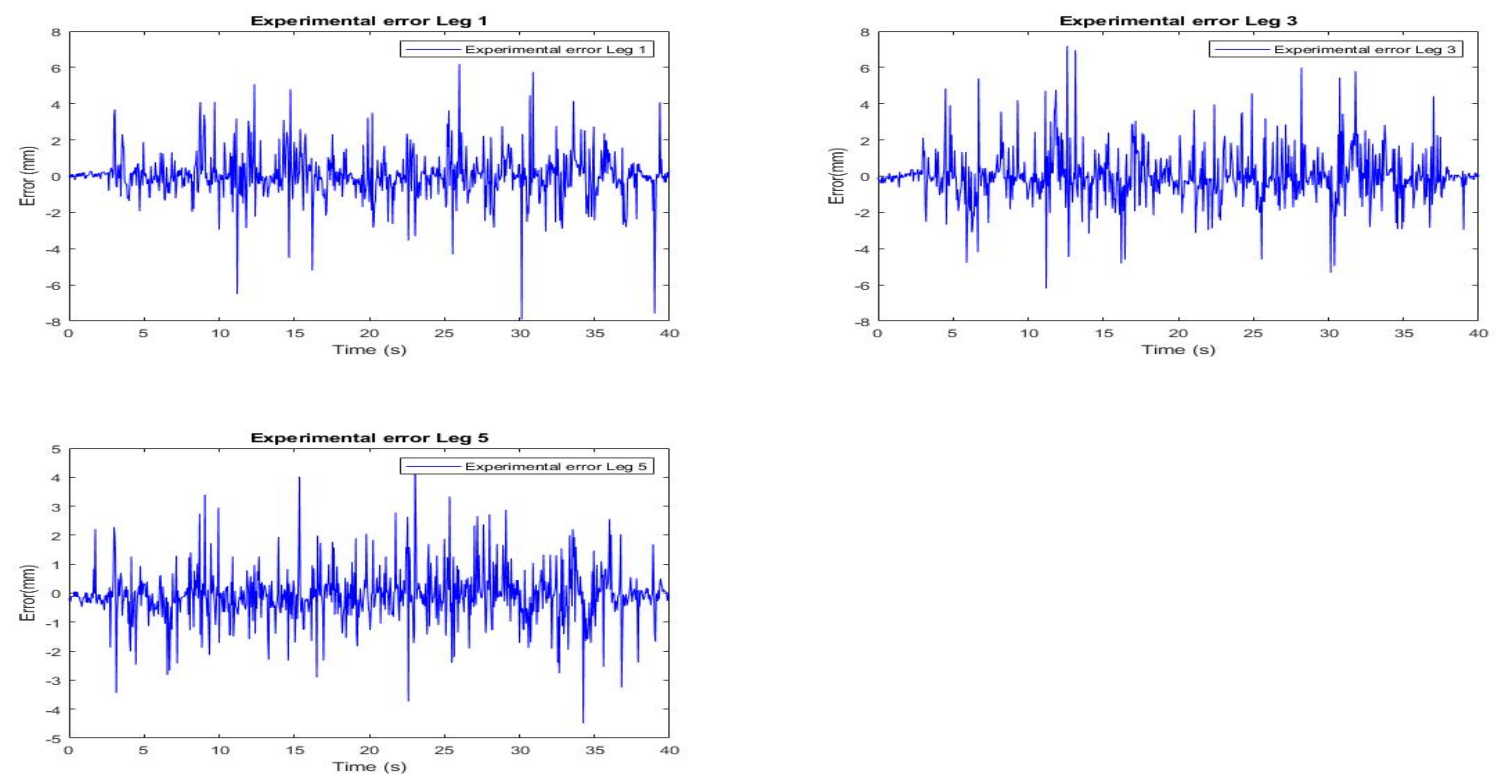

Figure 8-2: Default PD leg length error. 

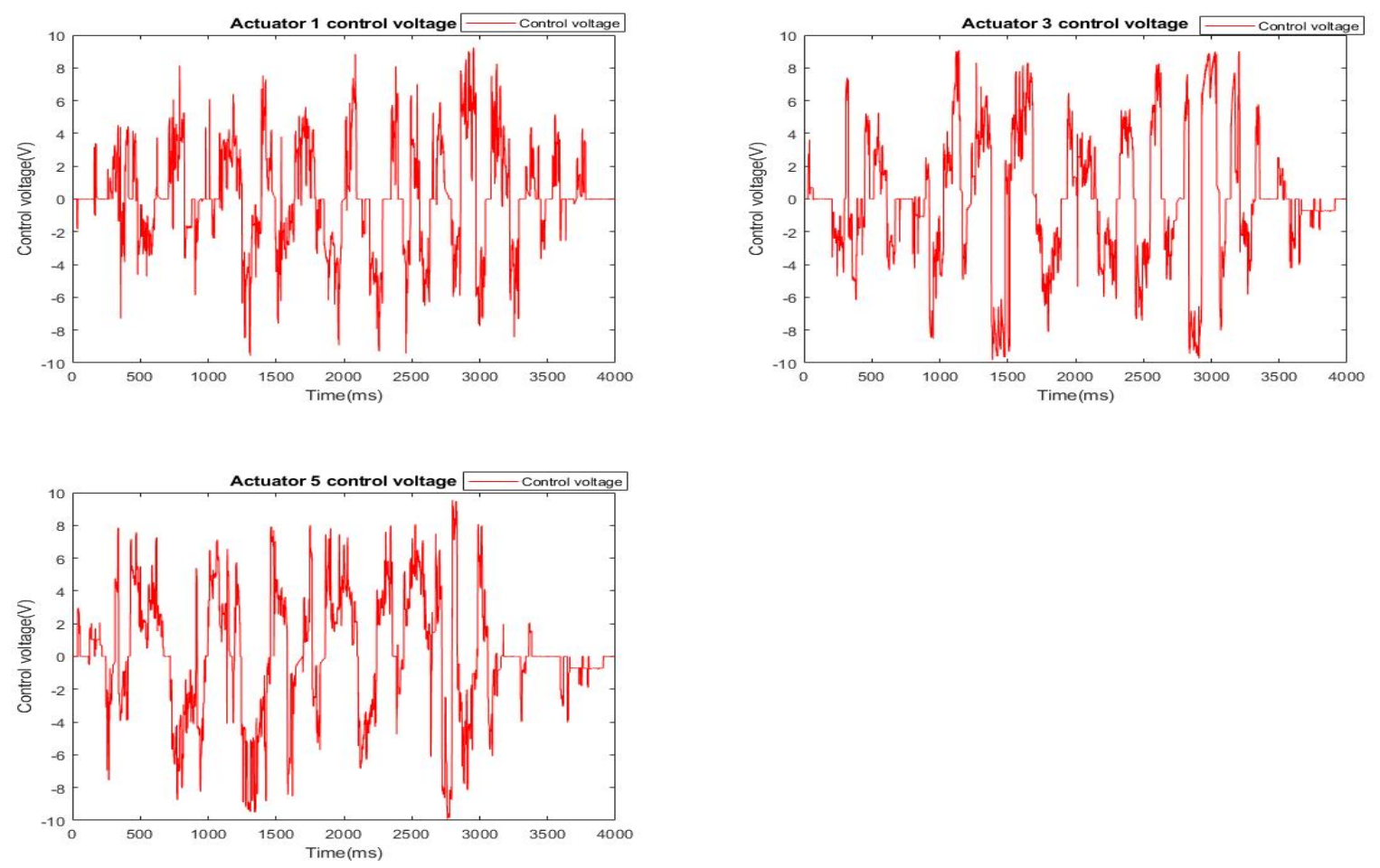

Figure 8-3: Actuator 1, 3 and 5 experimental control voltage.
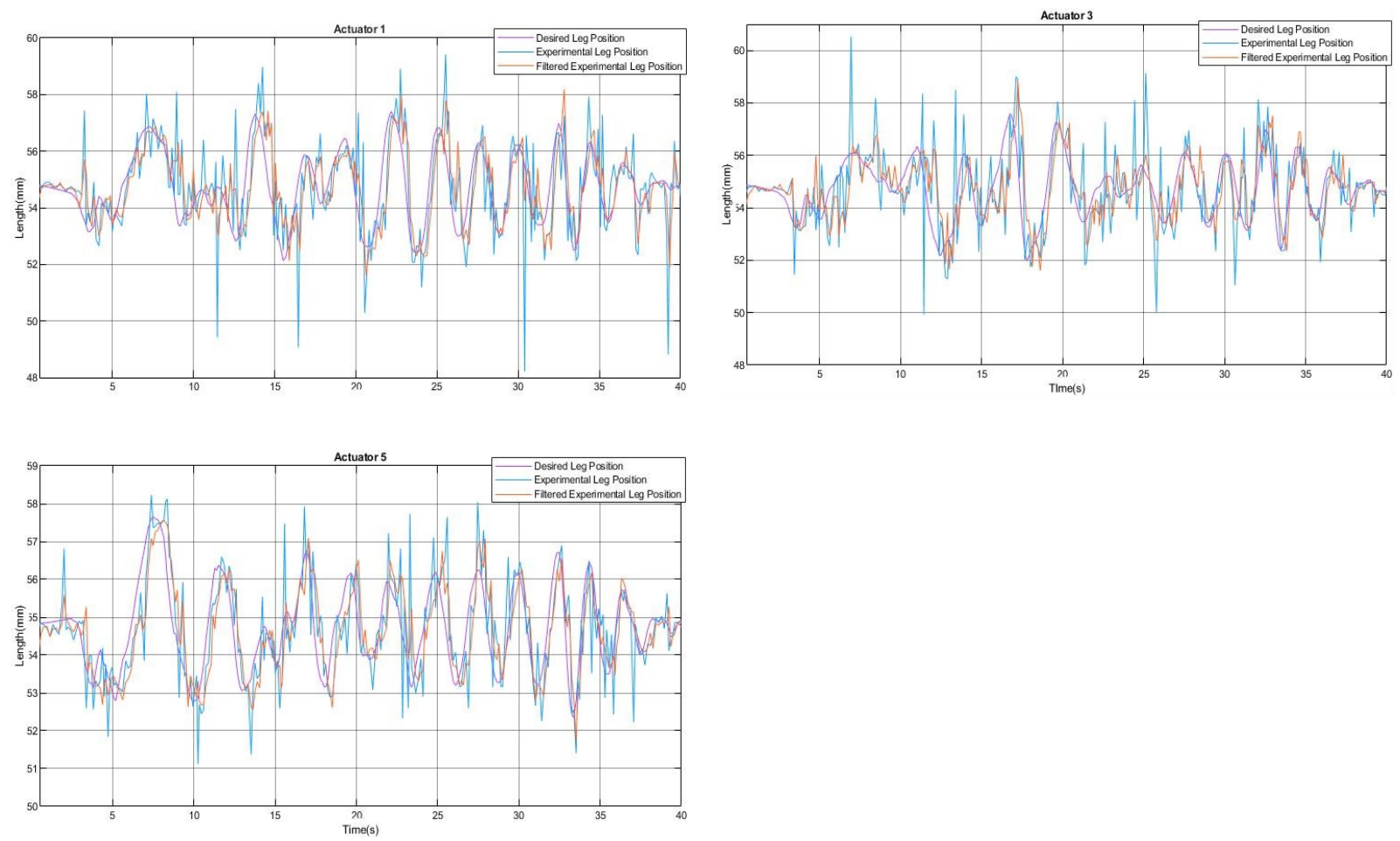

Figure 8-4: Proposed PD filtered experimental error. 


\subsection{Proposed PD experimental results}

The second experiment with the proposed PID controller was not possible to be tested on the SP due to the configuration in the utility software of the actuator and the lack of input power to the DC motors. Instead of that, a conservative control was proposed, based on the Bode plots and searching for an increment in the cut-off frequency and a phase gain. The proposed values for this control were $K_{p}=50$ and $K_{D}=50$.

The real position of the actuators can be compared with the desired position that was used as input to the SP. The resulting plots shown in Figure 8-5 display the desired against the measured position of the actuators 1, 3 and 5. The plots shown in Figure 8-6 show the position error also for actuators 1, 3 and 5. The control voltage plot is shown in Figure 8-7.

The measured positions using the actuator potentiometers is very noisy for each measurement; it was necessary to use a filter in order to obtain a more realistic result. After passing the data trough a first order filter with a time constant of $1 \mathrm{sec}$, the corresponding plots are shown in Fig. 8-8. The MSRE of each actuator is summarized in Table 8-2.

\begin{tabular}{|c|c|c|c|}
\hline & Leg 1 & Leg 3 & Leg 5 \\
\hline Proposed PD MSE & 0.9323 & 1.1551 & 0.3623 \\
\hline Default PD MSE & 1.1751 & 2.2415 & 0.4482 \\
\hline
\end{tabular}

Table 8-2: Proposed PD and Default PD MSE of actuators 1, 3 and 5 comparison. 

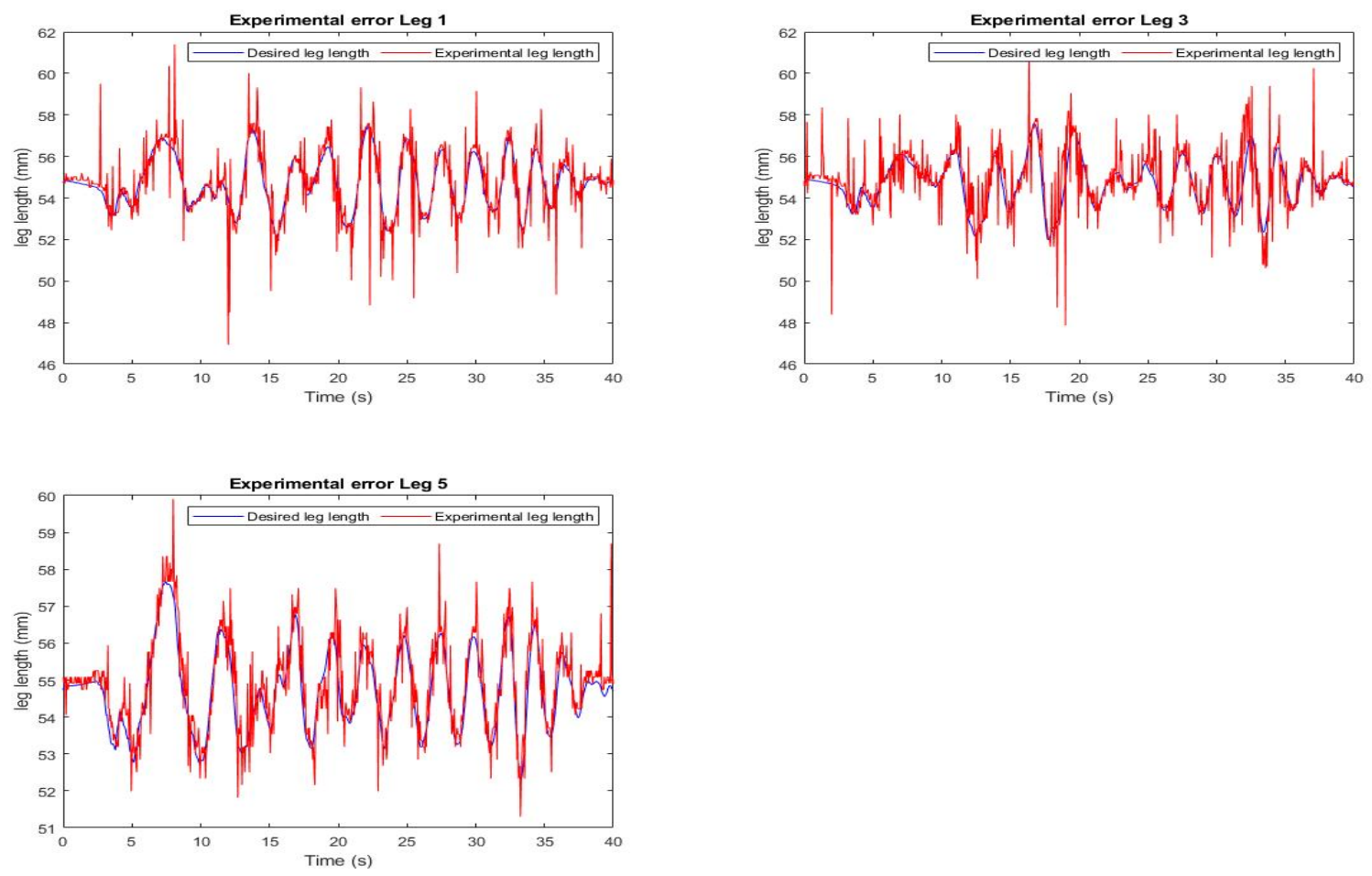

Figure 8-5: Proposed PD measured positions against desired positions.
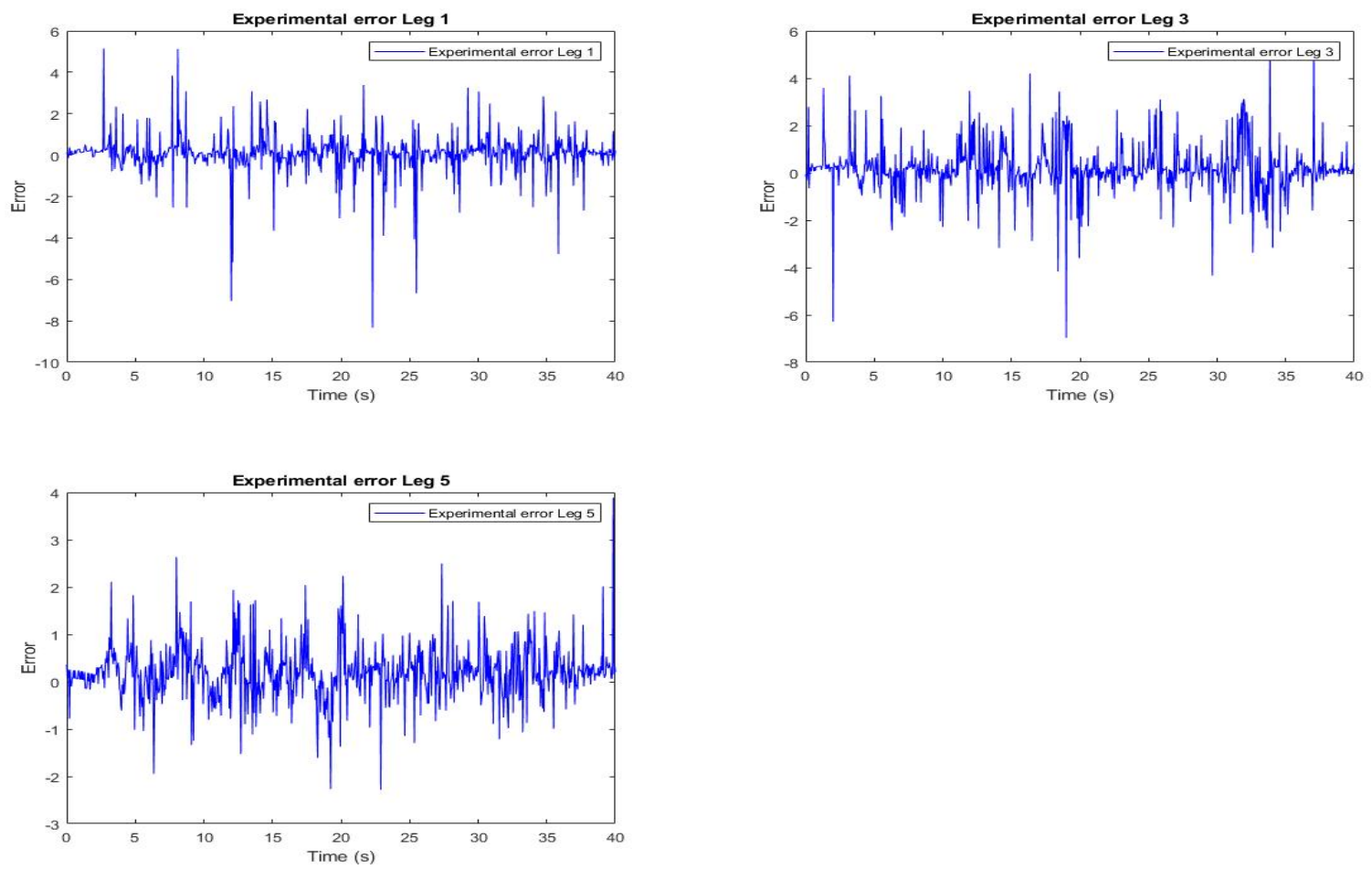

Figure 8-6: Proposed PD leg length error. 

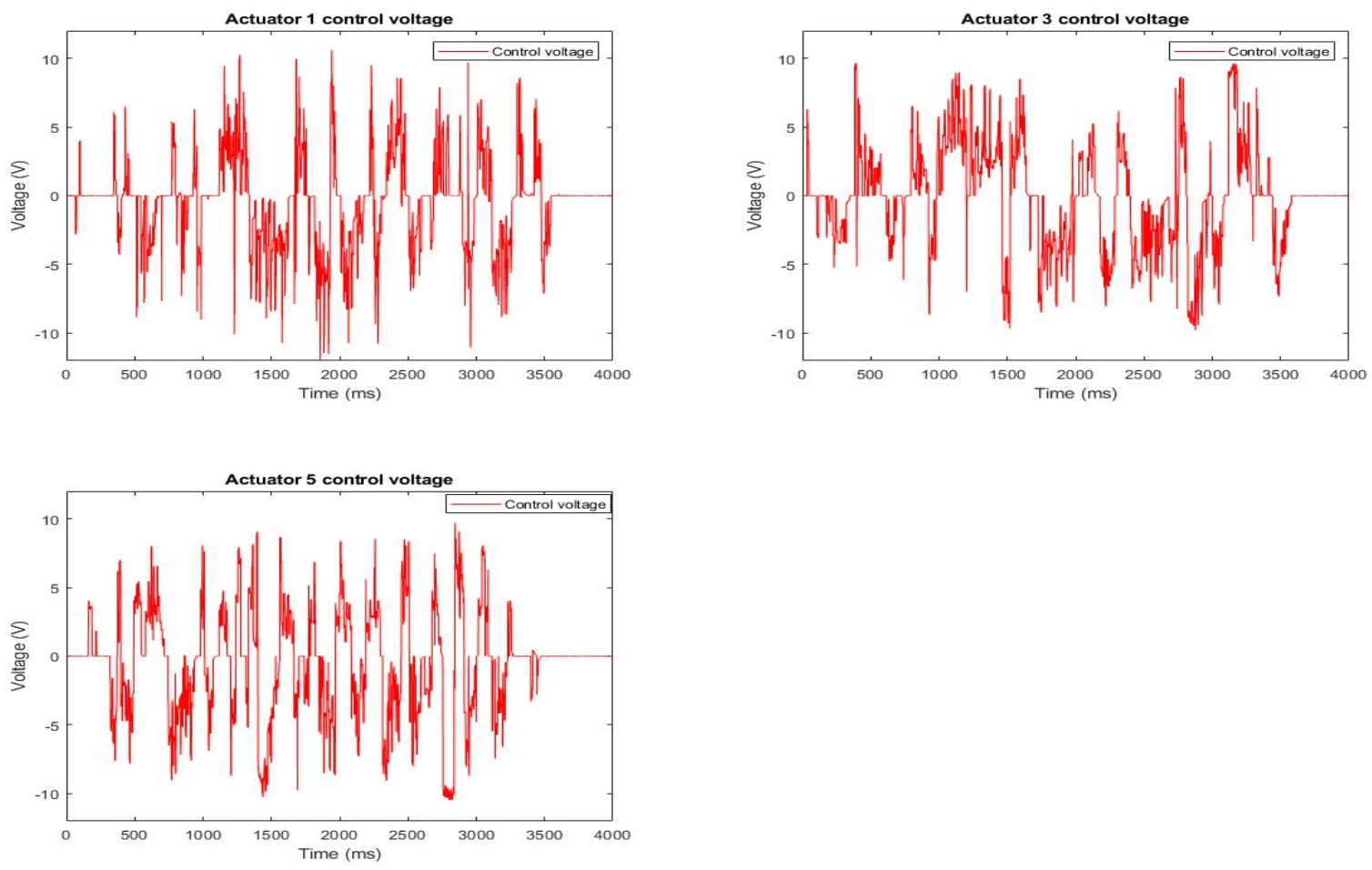

Figure 8-7: Proposed PD actuator 1, 3 and 5 experimental control voltage.
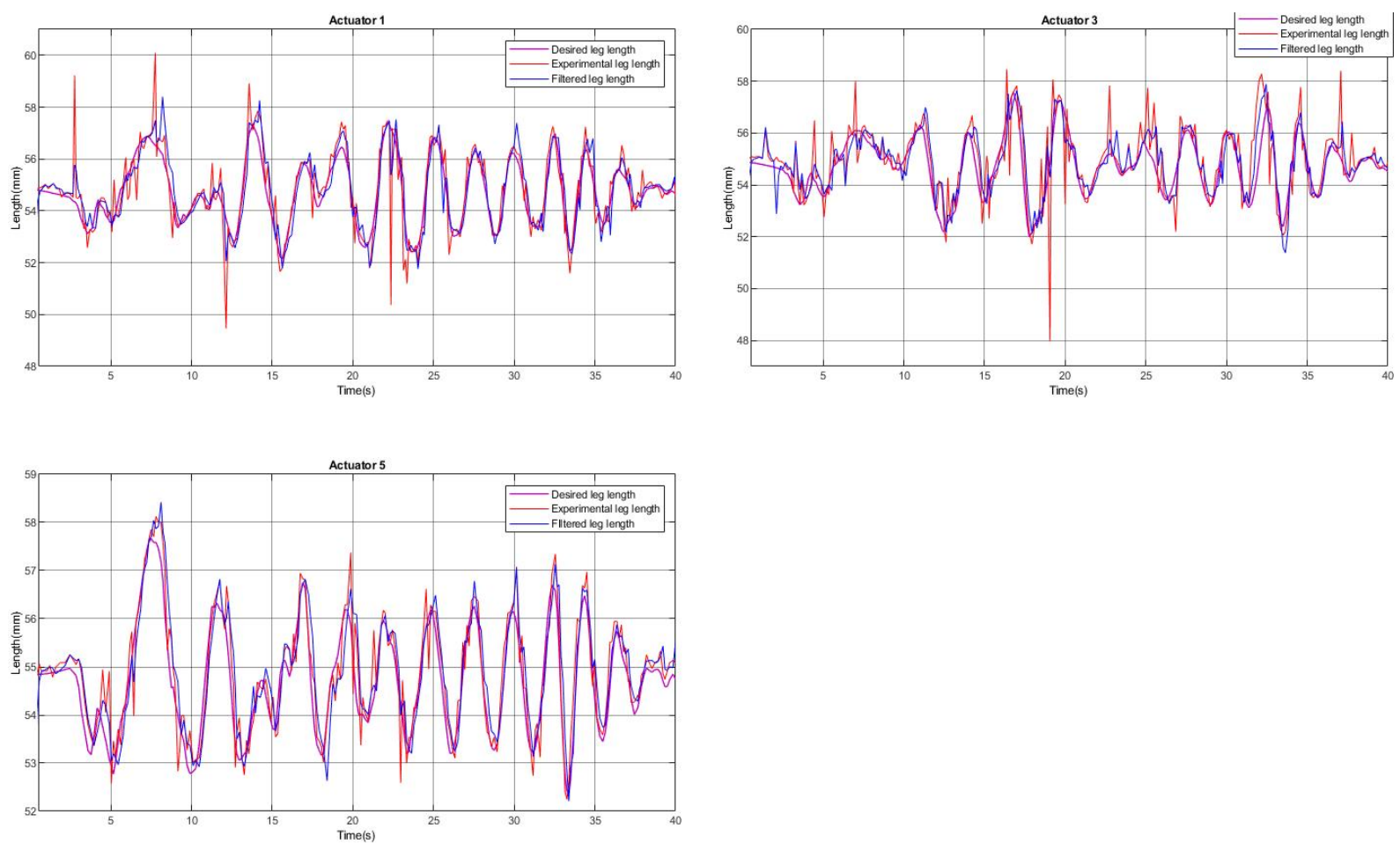

Figure 8-8: Proposed PD filtered experimental error. 


\section{Conclusions and Further Work}

The CIDESI hexapod is a serious robot project developed from scratch at CIDESI; thus, all the drawings and models were available in order to import them to Simscape, making the model as close as possible to the real one. Also few differences in part weights were taken into account. The DC motor that powers the actuators was modelled and added to the imported model, making it much closer to the real platform.

The simulation of the earthquake profile was slow due to the processing time of each position with the actuators, but the results were good. A very good improvement in the mean squared error in each actuator and axis was obtained, and this can be seen graphically in the resulting plots; also in the resulting plots we can observe that using the proposed PID controller, the control voltage was not saturating in any point of the routine.

For the experimental tests, it is evident that the measured position values at each point are following the reference values. Nevertheless, the error is quite larger than the results in simulation. Additionally, there is the problem of the noise in measurements of positions, this could be due to parasitic current that are returning to the voltage source when the routine is being performed; the filter that was applied help to obtain results closer to reality since the spikes on measured positions do not represent a real feasible motion of the platform. It is important that for further work to eliminate most of the noise generated in the measurements in order to be able to experiment with more earthquake "profiles" with different frequencies and have a better evaluation of the HxCf1 performance.

Measure of the output voltage to the motors was a little bit complicated due to the PWM that the driver uses; the signals for the channel A and the channel B of the motor do not have a specific order and when this signals are being obtained; due to the reason mentioned before, the measurements were filtered and the resulting plots are graphically different from the simulation plots, where the voltage measurement is directly in volts. Also, it is important to remark the importance of the power supplies, it is possible to see a difference between one power supply 
and another in the measurements of the position of the actuators.

The chosen earthquake ground motion profile was a good example due to the movements in the range of the millimetres, but it is important to test more with different frequencies and, if it is possible, recorded closer to México.

The default PD controller is a good starting point for certain applications and that is why the fabricator recommend it; in our specific application, the performance of the hexapod was improved with a proposed PD controller taking into account the characteristics of the Bode plot and testing the performance running the proposed earthquake routine.

For further work, an optimal inverse control can be applied, choosing four parameters to adjust the control performance $K, K_{P}, K_{I}$ and $\gamma$; the selection conditions of gains are suggested using the time derivative of Lyapunov function. This can lead to obtain an optimal performance of the SP. 


\section{Bibliography}

[1] The 10 most significant natural disasters worldwide by death toll from 1980 to 2018. https://www.statista.com/statistics/268029/natural-disastersby-death-toll-since-1980/. (accesed on 18-04-2019).

[2] Datasheet for glideforce light-duty linear actuators. https://www . pololu.com/file/ 0J1238/LD-Linear-Actuator-Data-Sheet.pdf. (accesed on 23-07-2019).

[3] Development of the number of earthquakes (m5+) worldwide from 2000 to 2017. https://www.statista.com/statistics/263105/development-of-thenumber-of-earthquakes-worldwide-since-2000/. (accesed on 18-04-2019).

[4] Digital tectonic activity map. https://visibleearth.nasa.gov/view.php?id= 88415. (accesed on 18-04-2019).

[5] Executive summary world robotics 2018 industrial robots robot sales. https: //ifr.org/downloads/press2018/Executive_Summary_WR_2018_Industrial_ Robots .pdf. (accesed on 016-04-2019).

[6] Fanuc r-2000ib product information sheet. https://www.fanucamerica.com/docs/ default-source/robotics-product-information-sheets/r-2000ib-series_ 177.pdf. (accesed on 16-04-2019).

[7] Global death toll due to earthquakes from 2000 to 2015. https: //www . statista.com/ statistics/263108/global-death-toll-due-to-earthquakes-since-2000/. (accesed on 18-04-2019).

[8] NEES shake table test gallery. https://www.strongtie.com/about/researchtesting-innovation/academia/nees-testing. (accesed on 16-04-2019).

[9] Pololu jrk usb motor controller user's guide. https ://www.pololu.com/docs/pdf/ 0J38/jrk_motor_controller.pdf. (accesed on 30-07-2019). 
[10] Technical Report for the PEER Ground Motion Database Web Application. https: //ngawest2.berkeley.edu/Technical_Report.pdf. (accesed on 19-04-2019).

[11] Users Manual for the PEER Ground Motion Database Web Application. https: //ngawest2.berkeley.edu/peer_ground_motion_database_users_manual.pdf. (accesed on 19-04-2019).

[12] Victor David Acosta-Abraham. Truck simulator mechanism development, preliminary stage. Technical report, CIDESI-CONACYT, 2016.

[13] Edgar Arciniega. Cinematic and dynamic simulations of CIDESI Hexapod HxCf1. Technical report, CIDESI, 2018.

[14] Marco Ceccarelli \& Erika Ottaviano \& Gianni Castelli. An application of a 3-dof parallel manipulator for earthquake simulations. 22nd International Symposium on Automation and Robotics in Construction, 2005.

[15] Youngjin Choi and Wan Kyun Chung. PID Trajectory Tracking Control for Mechanical Systems. Springer, 2004.

[16] Jose Ignacio Rojas Gregorio \& Emily BJ Coffey. Technology resources for earthquake monitoring and response (TREMOR), 2007.

[17] Christine Connolly. ABB high-speed picking robots establish themselves in food packaging. Industrial Robot, 34(4):281-284, 2007.

[18] Jhon J. Craig. Introduction To Robotics: Mechanics and Control. Addison Wesley Publishing Company, 1986.

[19] Y. X. Su \& B. Y. Duan. The application of the stewart platform in large spherical radio telescopes. Journal of Robotic Systems, 2000.

[20] Zoran Pandilov \& Vladimir Dukovski. Comparison of the characteristics between serial and parallel robots. Acta Technica Corviniensis - Bulletin of Engineering, 2014.

[21] José Luis Germán Félix and Gengis K. Toledo-Ramírez. Robot controller for a parallel robot, from conception to implementation. Master's thesis, CIDESI-CONACYT \& FH Aachen, 2018.

[22] Amir Ghobakhloo and M. Eghtesad. Closed form solution to inverse dynamics problem of a parallel robot (stewart platform) by lagrangian approach. Tehran international congress on manufacturing engineering (TICME2005), 2006. 
[23] Amir Ghobakhloo and M. Eghtesad. Position control of a stewart-gough platform using inverse dynamics method with full dynamics. Advanced Motion Control, 2006.

[24] Qimi Jiang \& Clément Gosselin. Singularity equations of gough-stewart platforms using a minimal set of geometric parameters. Journal of Mechanical Design, 2008.

[25] Tiare Hernandez-Aguirre and Gengis K. Toledo-Ramírez. Development of the "cidesi linear servoactuator". Master's thesis, CIDESI-CONACYT \& FH Aachen, 2018.

[26] Tian Chunyu \& Xiai Congzhen \&0 Zhang Hong. Shaking table test and seismic performance evaluation of shangai tower. International Jounal of High-Rise Buildings, 1(3):221-228, 2012.

[27] Barajas Fletes José Ramón \& Toledo Ramírez Gengis K. Diseño de detalle de prototipo alfa Hexápodo CIDESI - Fase I. Parte Mecánica. Technical report, CIDESI-CONACYT, 2018.

[28] Anders Lohmann Madsen \& Soren Giessing Kristensen. Design of stewart platform for wave compensation, 2012.

[29] Gerson Andrés Díaz López. Desarrollo de prototipo alfa de plataforma stewart-gough “Hexápodo CIDESI”. Technical report, CIDESI-CONACYT, 2018.

[30] J.-R MERLET. Determination of the orientation workspace of parallel manipulators. Journal of Intelligent and Robotic Systems, (13):143-160, 1995.

[31] J.P. Merlet. Parallel Robots. Springer, 2006.

[32] Richard M. Murray. A Mathematical Introduction to Robotic Manipulation. CRC Press, 1994.

[33] Ruben Ordaz-Madrigal. Diseño de plataforma stewart-gough "hexápodo-cidesi”, fase 1. pare mecánica. Technical report, CIDESI-CONACYT, 2017.

[34] Ernesto Salazar-Joya. Desarrollo de demo para robot hexapodo fanuc. Technical report, CIDESI, 2014.

[35] D. Stewart. A platform with six degrees of freedom. Proceedings of the Institution of Mechanical Engineers, pages 371-386, 1965.

[36] Gengis K. Toledo-Ramírez. Hexápodo-CIDESI, fase 1. conceptos de operación y requerimientos. Technical report, CIDESI-CONACYT, August 2018. 
[37] Lung-Wen Tsai. Robot Analysis- The Mechanics of Serial And Parallel Manipulators. John Wiley \& Sons, 1999.

[38] Aimee Goncalves Vatsal Patel, Sanjay Krishnan. Sprk: A low-cost stewart platform for motion study in surgical robotics. 2018 International Symposium on Medical Robotics (ISMR), 2017.

[39] Ayman El-Badawy \& Khaled Youssef. On modeling and simulation of 6 degrees of freedom stewart platform mechanism using multibody dynamics approach. ECCOMAS Multibody Dynamics, 2013.

[40] J.Wenlandt Y.VanWeddingen. Modeling flexible bodies with simscape multibody software. Technical report, Mathworks, 2017.

[41] J. G. Ziegler and N. B. Nichols. Optimum settings for automatic controllers. ASME Trans., 1942. 


\section{Acronyms}

CAD Computer Aided Design

CIDESI Centro de Ingeniería y Desarrollo Industrial

CLI Command Line Interface

CSV Comma Separated Values

DC Direct current

DGML Directed Graph Markup Language

DOF Degrees Of Freedom

DTAM Digital Tectonic Activity Map

GUI Graphic User Interface

HMI Human Machine Interface

HRRTCS High Reliability Real-Time Control Systems

IFR International Federation of Robotics

MSSM Minimal Symmetric Simplified Manipulator

NGA Next Generation of Ground-Motion Attenuation Models

PEER Pacific Earthquake Engineering Research center

PGMD PEER Ground Motion Database

PID Proportional Integral Derivative 
SP Stewart-Gough Platform

SSM Symmetric simplified Manipulator

TSSM Triangular Symmetric Simplified Manipulator 


\section{List of Figures}

1-1 Common used industrial robotic systems . . . . . . . . . . . . 2

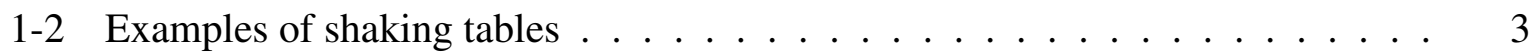

1-3 HxCf1 Hexapod CIDESI project . . . . . . . . . . . . . . . 3

1-4 Occurrence of Earthquakes Globally . . . . . . . . . . . . . . . . . 4

1-5 The 10 most significant natural disasters worldwide by death toll from 1980 to

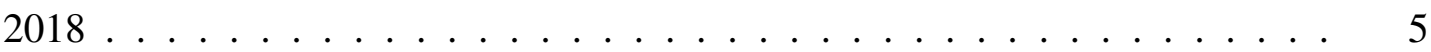

1-6 Global death toll due to earthquakes from 2000 to $2015 \ldots \ldots$. . . . . . . 5

1-7 Estimated annual worldwide supply of industrial robots 2009-2017 and 2018*-

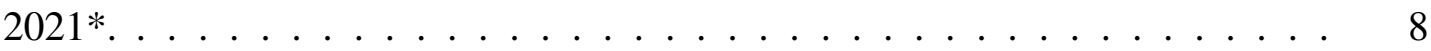

1-8 Estimated annual worldwide operational stock of industrial robots 2009-2017

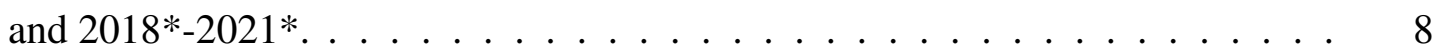

1-9 Development of the number of earthquakes (M5+) worldwide from 2000 to 20179

2-1 Stewart-Gough platform $\ldots \ldots \ldots \ldots \ldots$

2-2 Geometrical configurations for Stewart-Gough platforms of 6 DOF . . . . . . 14

2-3 Ship Crane mounted on Stewart platform . . . . . . . . . . . . . . 15

2-4 Proposed design structure of the fine tuning platform $\ldots \ldots \ldots \ldots$

2-5 Miniaturized Stewart platform to simulate anatomical movements . . . . . . 15

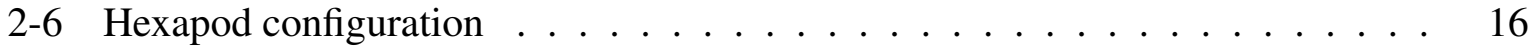

3-1 Assembled CIDESI Hexapod system . . . . . . . . . . . . . . . . 26

3-2 Assembly of critical components of the CIDESI Hexapod controller . . . . . 27

3-3 Robot electrical connections . . . . . . . . . . . . . . . . . . . . 28

4-1 CIDESI HxCf1 Hexapod. . . . . . . . . . . . . . . . . . . . . . 30

4-2 Angle characterization for the mobile platform using its coordinate system $T_{x y z} . \quad 30$

4-3 Schematic representation of the mobile platform. . . . . . . . . . . 31

4-4 Angle characterization for the base platform using its coordinate system $B_{x y z} . \quad$. 31 
4-5 Schematic representation of the base platform. . . . . . . . . . . . . . 32

4-6 Actuator velocity using higher possible frequency. . . . . . . . . . . . . . . 38

4-7 Actuator velocity using lower possible frequency. . . . . . . . . . . . . . . . 39

5-1 CIDESI HxCf1 Hexapod Simscape model . . . . . . . . . . . . . . . . . 42

5-2 CIDESI HxCf1 Hexapod exported geometry . . . . . . . . . . . . . . 43

5-3 CIDESI HxCf1 Hexapod visual output . . . . . . . . . . . . . . . . 43

5-4 PGMD search engine interface. . . . . . . . . . . . . . . . 45

5-5 Sparks earthquake information. . . . . . . . . . . . . . 46

5-6 Earthquake components time series for acceleration, velocity and position. . . . 47

$5-7$ Simscape DC motor model. . . . . . . . . . . . . . . . . . . 5 51

$5-8$ Simscape DC motor model simulation. . . . . . . . . . . . . . . . 51

$5-9$ Linear actuator performance data. . . . . . . . . . . . . . . 52

6-1 Closed loop DC motor model. . . . . . . . . . . . . . . . . 55

6-2 Default PD trajectory tracking performance. . . . . . . . . . . . . 55

6-3 Default PD open loop Bode plot. . . . . . . . . . . . . . . . . . 55

$6-4$ Plant Bode plot. . . . . . . . . . . . . . . . . 56

6-5 Tuned PID trajectory tracking performance. . . . . . . . . . . 58

$6-6$ Tuned PID Bode plot. . . . . . . . . . . . . . . . . 58

7-1 PD desired against real actuator Length. . . . . . . . . . . . . . . . . 60

$7-2 \quad$ PD leg length error. . . . . . . . . . . . . . . . . . . 61

7-3 PD mobile platform axis position. . . . . . . . . . . . . . 62

7-4 Default PD mobile platform axis error. . . . . . . . . . . . . . . 62

$7-5$ PID control voltage for each actuator. . . . . . . . . . . . . 63

7-6 PID desired against real actuator Length. . . . . . . . . . . . . . . . . 65

$7-7$ PID leg length error. . . . . . . . . . . . . . . . . 66

$7-8$ PID mobile platform axis position. . . . . . . . . . . . . 67

7-9 PID mobile platform axis error. . . . . . . . . . . . . . . 67

7-10 PID control voltage for each actuator. . . . . . . . . . . . . 68

8-1 Default PD actuator measured positions against desired positions. . . . . . . . 70

8-2 Default PD leg length error. . . . . . . . . . . . . . . . 70

8-3 Actuator 1, 3 and 5 experimental control voltage. . . . . . . . . . . . . 71

8-4 Proposed PD filtered experimental error. . . . . . . . . . . . . . . 71

8-5 Proposed PD measured positions against desired positions. . . . . . . . . . . . 73

8-6 Proposed PD leg length error. . . . . . . . . . . . . . . . . 73 
8-7 Proposed PD actuator 1, 3 and 5 experimental control voltage. . . . . . . . . . 74

8-8 Proposed PD filtered experimental error. . . . . . . . . . . . . . . . . 74

A-1 Chronogram. . . . . . . . . . . . . . . . . 89 



\section{List of Tables}

3-1 Development phases for the CIDESI Hexapod project . . . . . . . . . . 23

4-1 Physical parameters of HxCf1 Hexapod . . . . . . . . . . . . . . . . 32

4-2 Point coordinates for $B_{i}$ and $G T_{i}$. . . . . . . . . . . . . . . . . . . 33

$5-1 \quad$ Linear actuator parameters. . . . . . . . . . . . . . . . . . 47

$5-2$ Linear actuator found parameters. . . . . . . . . . . . . . . 50

5-3 Rotor, screw parameters and combined inertia. . . . . . . . . . . . 50

6-1 Tuning formulas for $K_{p}, K_{I}$ and $K_{D} \ldots \ldots \ldots \ldots \ldots$

7-1 PD MSE of actuators and mobile platform. . . . . . . . . . . . . . . . 60

7-2 PID MSE of actuators and mobile platform. . . . . . . . . . . . . . 64

8-1 PD MSE of actuators 1,3 and $5 \ldots \ldots \ldots$

8-2 Proposed PD and Default PD MSE of actuators 1, 3 and 5 comparison. . . . . . 72 



\section{A Chronogram}

The main activities required from the start to the finish, or completion of the Thesis are represented in the Gantt chart shown in the following Choronogram.

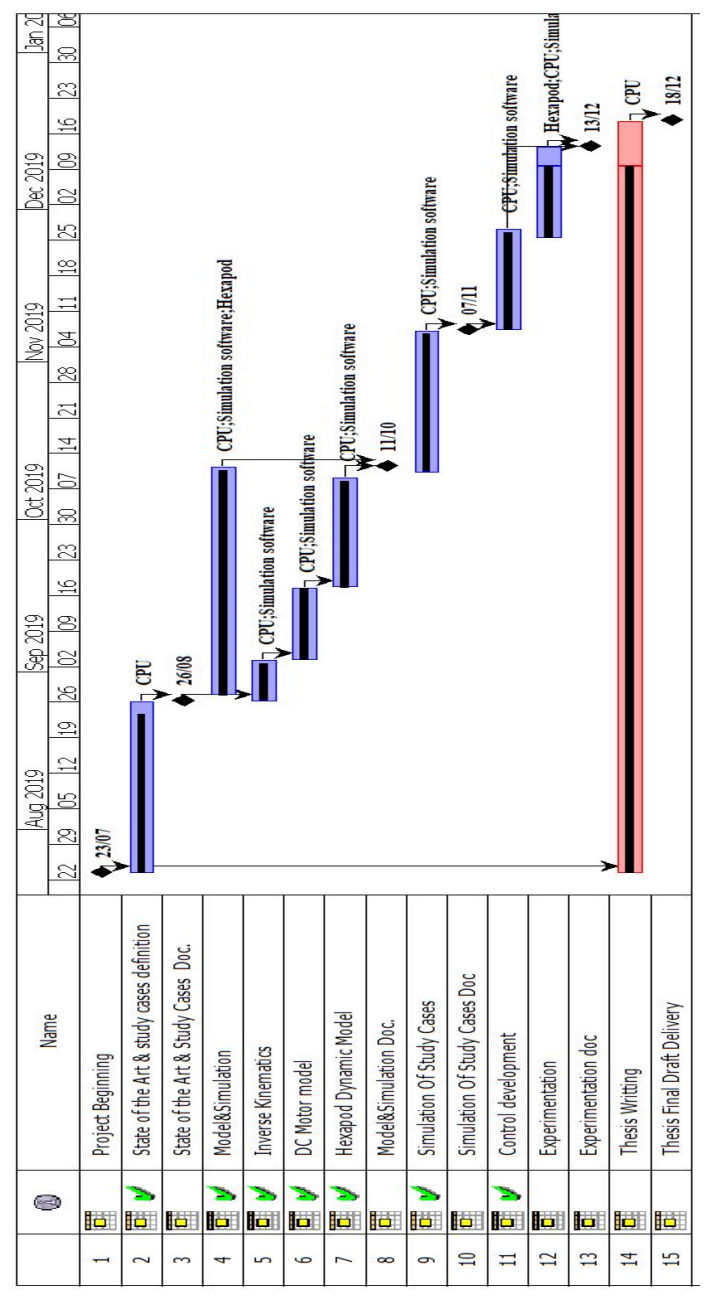

Figure A-1: Chronogram. 



\section{B Positions and Orientations}

function $\mathrm{y} 1=\mathrm{fcn}(\mathrm{x}, \mathrm{y}, \mathrm{z}, \mathrm{a}, \mathrm{b}, \mathrm{g})$

(InTech Hexapod)

$\mathrm{Rp}=94.33987595$;

$\mathrm{Rb}=146.6330849$;

$\mathrm{Tp}=25.68141134$;

$\mathrm{Tb}=16.41456206$;

Measures of Hexapod-CIDESI

Max Value of Z-max=465.43mm, Z-min=345.02mm

(Actuators Length; L-max 481.8244and L-min 366.83)

$\mathrm{Rp}=192.86$; Mobile Platform Radius

$\mathrm{Rb}=247.97$; Base Platform Radius

$\mathrm{Tp}=97.88 * \mathrm{p}$ / 180 ; Theta P. Separation Mobile Platform

$\mathrm{Tb}=20.4414 * \mathrm{p} / \mathrm{180}$; Theta B. Base Mobile Platform

Rotation Matrix

$\mathrm{r} 11=\cos (\mathrm{b}) * \cos (\mathrm{g})$;

$\mathrm{r} 12=\cos (\mathrm{g}) * \sin (\mathrm{a}) * \sin (\mathrm{b})-\cos (\mathrm{a}) * \sin (\mathrm{g}) ;$

$\mathrm{r} 13=\sin (\mathrm{a}) * \sin (\mathrm{g})+\cos (\mathrm{a}) * \cos (\mathrm{g}) * \sin (\mathrm{b})$;

$\mathrm{r} 21=\cos (\mathrm{b}) * \sin (\mathrm{g})$;

$\mathrm{r} 22=\cos (\mathrm{a}) * \cos (\mathrm{g})+\sin (\mathrm{a}) * \sin (\mathrm{b}) * \sin (\mathrm{g}) ;$ 
$\mathrm{r} 23=\cos (\mathrm{a}) * \sin (\mathrm{b}) * \sin (\mathrm{g})-\cos (\mathrm{g}) * \sin (\mathrm{a})$

r31=- $\sin (b)$;

$\mathrm{r} 32=\cos (\mathrm{b})^{*} \sin (\mathrm{a})$

$\mathrm{r} 33=\cos (\mathrm{a}) * \cos (\mathrm{b})$;

Position Vector

$\mathrm{P}=[\mathrm{x} ; \mathrm{y} ; \mathrm{z}] ; \mathrm{Px}=\mathrm{P}(1) ; \mathrm{Py}=\mathrm{P}(2) ; \mathrm{Pz}=\mathrm{P}(3)$;

Mobile Platform

Actuators for $\mathrm{i}(1,3,5)$

Lambda1 $=(1 * \mathrm{pi} / 3-\mathrm{Tp} / 2)$;

Lambda3=(3*pi/3-Tp/2);

Lambda5=(5* pi/3-Tp/2);

Actuators for 2,4,6

Lambda2=(Lambda1+Tp $)$;

Lambda4=(Lambda3+Tp $)$;

Lambda6=(Lambda5+Tp $)$;

Fixed Base

Actuators for $\mathrm{i}(1,3,5)$

Ipsilon $1=(1 * \mathrm{pi} / 3-\mathrm{Tb} / 2)$;

Ipsilon3=(3*pi/3-Tb/2);

Ipsilon5=(5* pi/3-Tb/2);

Actuators for i $(2,4,6)$

Ipsilon2=(Ipsilon $1+\mathrm{Tb})$;

Ipsilon4=(Ipsilon3+Tb);

Ipsilon6=(Ipsilon5+Tb);

Column Vector M.P. GTi

Actuators for 1,3,5 
$\mathrm{GT} 1=\left[\mathrm{Rp} * \cos (\right.$ Lambda1 $) ; \mathrm{Rp}^{*} \sin ($ Lambda1);0];

GT3 $=\left[\right.$ Rp* $\cos ($ Lambda3 $) ; \mathrm{Rp}^{*} \sin ($ Lambda3 $\left.) ; 0\right]$;

GT5 $=\left[\mathrm{Rp}^{*} \cos (\right.$ Lambda5);Rp*sin(Lambda5);0];

Actuators for $2,4,6$

GT2 $=[$ Rp* $\cos ($ Lambda2);Rp*sin $($ Lambda2);0];

GT4=[Rp* $\cos ($ Lambda4);Rp*sin(Lambda4);0];

GT6 $=[$ Rp* $\cos ($ Lambda6);Rp*sin $($ Lambda6);0];

Column Vector for Fixed Base

Actuators for $1,3,5$

$\mathrm{B} 1=\left[\mathrm{Rb}^{*} \cos (\operatorname{Ipsilon} 1) ; \mathrm{Rb}^{*} \sin (\right.$ Ipsilon 1$\left.) ; 0\right]$;

$\mathrm{B} 3=\left[\mathrm{Rb}^{*} \cos (\right.$ Ipsilon3); $\mathrm{Rb} * \sin ($ Ipsilon3 $) ; 0]$;

$\mathrm{B} 5=\left[\mathrm{Rb}^{*} \cos (\right.$ Ipsilon5); $\mathrm{Rb} * \sin ($ Ipsilon5);0];

Actuators for 2,4,6

$\mathrm{B} 2=\left[\mathrm{Rb}^{*} \cos (\right.$ Ipsilon2); $\mathrm{Rb} * \sin ($ Ipsilon2);0];

$\mathrm{B} 4=\left[\mathrm{Rb}^{*} \cos \left(\right.\right.$ Ipsilon4); $\mathrm{Rb}^{*} \sin ($ Ipsilon4);0];

$\mathrm{B} 6=\left[\mathrm{Rb}^{*} \cos (\right.$ Ipsilon6);Rb* $\sin ($ Ipsilon6);0];

Rotation Matrix

Rxyz=[r11 r12 r13;r21 r22 r23;r31 r32 r33];

Resulting vectors

Actuators 1,3,5

$\mathrm{L} 1=\mathrm{Rxyz} * \mathrm{GT} 1+\mathrm{P}-\mathrm{B} 1$;

$\mathrm{L} 3=\mathrm{Rxyz} * \mathrm{GT} 3+\mathrm{P}-\mathrm{B} 3$;

$\mathrm{L} 5=\mathrm{Rxyz} * \mathrm{GT} 5+\mathrm{P}-\mathrm{B} 5$;

Actuators 2,4,6

$\mathrm{L} 2=\mathrm{Rxyz} * \mathrm{GT} 2+\mathrm{P}-\mathrm{B} 2$;

L4=Rxyz*GT4+P-B4; 


\section{L6=Rxyz*GT6+P-B6; \\ $\mathrm{D} 1=\mathrm{Rxyz} * \mathrm{GT} 1$; \\ D3=Rxyz*GT3; \\ D5=Rxyz $*$ GT5;}

Actuadores 2,4,6

D2=Rxyz*GT2;

D4=Rxyz*GT4;

D6=Rxyz*GT6;

y1 = $[\operatorname{norm}(\mathrm{L} 1) \operatorname{norm}(\mathrm{L} 2) \operatorname{norm}(\mathrm{L} 3)$ norm(L4) norm(L5) norm(L6)]; 


\section{Velocities}

function $\mathrm{y} 2=\mathrm{fcn}(\mathrm{x}, \mathrm{y}, \mathrm{z}, \mathrm{a}, \mathrm{b}, \mathrm{g}, \mathrm{vx}, \mathrm{vy}, \mathrm{vz}, \mathrm{va}, \mathrm{vb}, \mathrm{vg})$

Measures of Hexapod-CIDESI

Max Value of Z-max=465.43mm, Z-min=345.02mm

(Actuators Length; L-max 481.8244and L-min 366.83)

$\mathrm{Rp}=192.86$; Mobile Platform Radius

$\mathrm{Rb}=247.97$; Base Platform Radius

$\mathrm{Tp}=97.88 * \mathrm{p} i / 180$; Theta P. Separation Mobile Platform

$\mathrm{Tb}=20.4414 *$ pi/180; Theta B. Base Mobile Platform

Rotation Matrix values

$\mathrm{r} 11=\cos (\mathrm{b})^{*} \cos (\mathrm{g})$;

$\mathrm{r} 12=\cos (\mathrm{g}) * \sin (\mathrm{a}) * \sin (\mathrm{b})-\cos (\mathrm{a}) * \sin (\mathrm{g}) ;$

$\mathrm{r} 13=\sin (\mathrm{a}) * \sin (\mathrm{g})+\cos (\mathrm{a}) * \cos (\mathrm{g}) * \sin (\mathrm{b}) ;$

$\mathrm{r} 21=\cos (\mathrm{b}) * \sin (\mathrm{g})$;

$\mathrm{r} 22=\cos (\mathrm{a}) * \cos (\mathrm{g})+\sin (\mathrm{a}) * \sin (\mathrm{b}) * \sin (\mathrm{g}) ;$

$\mathrm{r} 23=\cos (\mathrm{a}) * \sin (\mathrm{b}) * \sin (\mathrm{g})-\cos (\mathrm{g}) * \sin (\mathrm{a})$;

$\mathrm{r} 31=-\sin (b)$;

$\mathrm{r} 32=\cos (\mathrm{b}) * \sin (\mathrm{a})$;

$\mathrm{r} 33=\cos (\mathrm{a}) * \cos (\mathrm{b})$

Position and orientation matrix 
Rxyz=[r11 r12 r13;r21 r22 r23;r31 r32 r33];

Position vector $(\mathrm{P})$

$\mathrm{P}=[\mathrm{x} ; \mathrm{y} ; \mathrm{z}]$;

First derivative of the positions

$\mathrm{Dx}=\mathrm{vx}$

$\mathrm{Dy}=\mathrm{vy}$;

$\mathrm{Dz}=\mathrm{vz}$

$\mathrm{Dxyz}=[\mathrm{Dx} ; \mathrm{Dy} ; \mathrm{Dz}]$;

First derivative of the orientations

$\mathrm{Da}=\mathrm{va}$;

$\mathrm{Db}=\mathrm{vb}$

$\mathrm{Dg}=\mathrm{vg}$;

$\mathrm{Dabg}=[\mathrm{Da} ; \mathrm{Db} ; \mathrm{Dg}] ;$

Angular velocities matrix assignations

$\mathrm{w} 11=\cos (\mathrm{b}) * \cos (\mathrm{g})$;

$\mathrm{w} 12=-\sin (\mathrm{g})$;

w13=0;

$\mathrm{w} 21=\cos (\mathrm{b}) * \sin (\mathrm{g})$;

$\mathrm{w} 22=\cos (\mathrm{g})$;

$w 23=0$;

$\mathrm{w} 31=\sin (b)$;

w32=0;

w33=-1;

Angular velocity matrix

$\mathrm{Wxyz}=[\mathrm{w} 11 \mathrm{w} 12 \mathrm{w} 13 ; \mathrm{w} 21 \mathrm{w} 22 \mathrm{w} 23 ; \mathrm{w} 31 \mathrm{w} 32 \mathrm{w} 33]$;

Angular velocity 
$\mathrm{W}=\mathrm{Wxyz} * \mathrm{Dabg}$;

Mobile base

Actuators i(1,3,5)

Lambda $1=(1 * \mathrm{pi} / 3-\mathrm{Tp} / 2)$;

Lambda3=(3*pi/3-Tp/2);

Lambda $5=\left(5^{*} \mathrm{pi} / 3-\mathrm{Tp} / 2\right)$;

Actuators 2,4,6

Lambda2 $=($ Lambda1 + Tp $)$;

Lambda4=(Lambda3+Tp);

Lambda6=(Lambda5+Tp $)$;

Fixed base

Actuators i $(1,3,5)$

Ipsilon $1=(1 * \mathrm{pi} / 3-\mathrm{Tb} / 2)$;

Ipsilon $3=(3 * \mathrm{pi} / 3-\mathrm{Tb} / 2)$;

Ipsilon $5=(5 * \mathrm{pi} / 3-\mathrm{Tb} / 2)$;

Actuators 2,4,6

Ipsilon2= $($ Ipsilon $1+\mathrm{Tb})$;

Ipsilon4=(Ipsilon3+Tb);

Ipsilon6=(Ipsilon5+Tb $)$;

Gti equation for the mobile platform

Actuadores 1,3,5

$\mathrm{GT} 1=\left[\mathrm{Rp}{ }^{*} \cos (\right.$ Lambda1 $) ; \mathrm{Rp}^{*} \sin ($ Lambda1);0];

GT3=[Rp* $\cos ($ Lambda3);Rp*sin(Lambda3);0];

GT5 $=\left[\mathrm{Rp}^{*} \cos (\right.$ Lambda5);Rp*sin(Lambda5);0];

Actuators 2,4,6

$\mathrm{GT} 2=\left[\mathrm{Rp} * \cos (\right.$ Lambda2 $) ; \mathrm{Rp}^{*} \sin ($ Lambda2 $\left.) ; 0\right]$; 
GT4=[Rp*cos(Lambda4);Rp*sin(Lambda4);0];

GT6=[Rp*cos(Lambda6);Rp*sin(Lambda6);0];

Bi equation for the fixed platform

Actuators 1,3,5

$\mathrm{B} 1=\left[\mathrm{Rb}^{*} \cos (\operatorname{Ipsilon} 1) ; \mathrm{Rb}^{*} \sin (\operatorname{Ipsilon} 1) ; 0\right]$;

$\mathrm{B} 3=\left[\mathrm{Rb} * \cos (\operatorname{Ipsilon} 3) ; \mathrm{Rb}^{*} \sin (\operatorname{Ipsilon} 3) ; 0\right]$;

$\mathrm{B} 5=\left[\mathrm{Rb}^{*} \cos \left(\right.\right.$ Ipsilon5); $\mathrm{Rb}^{*} \sin ($ Ipsilon5);0];

Actuators 2,4,6

$\mathrm{B} 2=\left[\mathrm{Rb}{ }^{*} \cos \left(\right.\right.$ Ipsilon2); $\mathrm{Rb}^{*} \sin ($ Ipsilon2);0];

$\mathrm{B} 4=\left[\mathrm{Rb}^{*} \cos (\right.$ Ipsilon4); Rb* $\sin ($ Ipsilon4);0];

$\mathrm{B} 6=\left[\mathrm{Rb}^{*} \cos \left(\right.\right.$ Ipsilon6); $\mathrm{Rb}^{*} \sin ($ Ipsilon6);0];

Length resulting vectors

Actuators 1,3,5

$\mathrm{L} 1=\mathrm{R} x \mathrm{yz} * \mathrm{GT} 1+\mathrm{P}-\mathrm{B} 1$;

L3=Rxyz*GT3+P-B3;

$\mathrm{L} 5=\mathrm{Rxyz} * \mathrm{GT} 5+\mathrm{P}-\mathrm{B} 5$;

Actuators 2,4,6

$\mathrm{L} 2=\mathrm{Rxyz} * \mathrm{GT} 2+\mathrm{P}-\mathrm{B} 2$;

L4=Rxyz*GT4+P-B4;

L6=Rxyz*GT6+P-B6;

Unitary vector ui

$\mathrm{u} 1=\mathrm{L} 1 / \operatorname{norm}(\mathrm{L} 1)$;

u2=L2/norm(L2);

u3=L3/norm(L3);

u4=L4/norm(L4);

u5=L5/norm(L5); 
u6=L6/norm(L6);

Velocity in point $\mathrm{Tj}$

$\mathrm{j} 1=\mathrm{Rxyz} * \mathrm{GT} 1$;

j2=Rxyz*GT2;

j3=Rxyz*GT3;

j4=Rxyz*GT4;

j5=Rxyz*GT5;

j6=Rxyz*GT6;

Velocity vector

VTj1=Dxyz+cross $(W, j 1)$;

VTj2=Dxyz+cross $(W, j 2)$;

VTj3=Dxyz+cross(W,j3);

VTj4=Dxyz+cross(W,j4);

VTj5=Dxyz+cross $(W, j 5)$;

VTj6=Dxyz+cross(W,j6);

Velocity in each leg

$\mathrm{VL} 1=\mathrm{VTj} 1{ }^{\prime} * \mathrm{u} 1$;

$\mathrm{VL} 2=\mathrm{VTj} 2{ }^{\prime}{ }^{\mathrm{u}} 2$;

$\mathrm{VL} 3=\mathrm{VTj}^{\prime}{ }^{*} \mathrm{u} 3$;

$\mathrm{VL} 4=\mathrm{VTj} 4{ }^{\prime} * \mathrm{u} 4$;

$\mathrm{VL5}=\mathrm{VTj} 5 * \mathrm{u} 5$;

$\mathrm{VL6}=\mathrm{VTj} 6{ }^{*} * \mathrm{u} 6$

y2 = [VL1 VL2 VL3 VL4 VL5 VL6]; 

D Pololu LACT6P linear actuator datasheet 


\section{$\Gamma$

\section{Product Data Sheet}

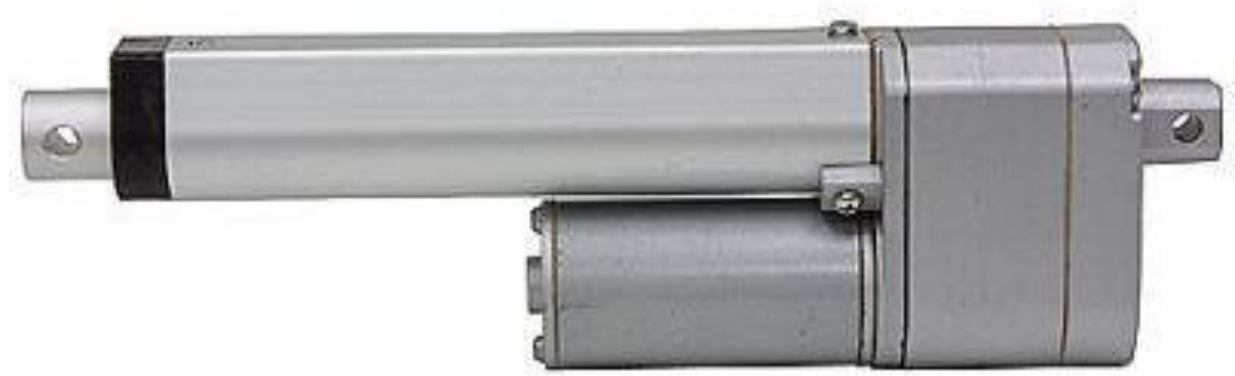

\section{Standard Features:}

- Compact design

- All aluminum frame and extension tube

- Nominal stroke lengths: 2" [50mm], 4" [100mm], 6" [150mm], 8" [200mm], $10 "[250 \mathrm{~mm}], 12 "[300 \mathrm{~mm}]$

- Input voltage: 12VDC, 24VDC

- Standard gear ratio: 20:1 [other ratios available]

- Rated load range: 34 lbs. [150 Newtons] -to- 225 lbs. [1000 Newtons]

- Maximum static load: 562 lbs. [2,500 Newtons]

- Maximum current draw: 3.2 Amps @ 12 VDC

- Speed at no load: $0.28 \mathrm{in} / \mathrm{sec}[7 \mathrm{~mm} / \mathrm{sec}]-t o-1.73 \mathrm{in} / \mathrm{sec}[43.9 \mathrm{~mm} / \mathrm{sec}]$

- Speed at full load: $0.22 \mathrm{in} / \mathrm{sec}$ [5.5 mm/sec] -to- $1.44 \mathrm{in} / \mathrm{sec}$ [36.5 mm/sec]

- Standard preset limit switches at both ends of stroke

- Noise level: $\leq 70 \mathrm{~dB}$

- Mounting hole diameter (both ends): 1/4" [6.4mm]

- Duty cycle: $25 \%$ or 1 min continuous operation in $4 \mathrm{~min}$.

- Working temperature: $-13 \mathrm{~F}[-25 \mathrm{C}]+149 \mathrm{~F}[+65 \mathrm{C}]$

- IP rating: IP65

\section{Accessories:}

- Mounting brackets

- Direction reversing switches

- Wired and wireless controllers

- Direction reversing relays 


\section{F GLIDEFORCE}

\section{Dimensions - Actuator Without Potentiometer}
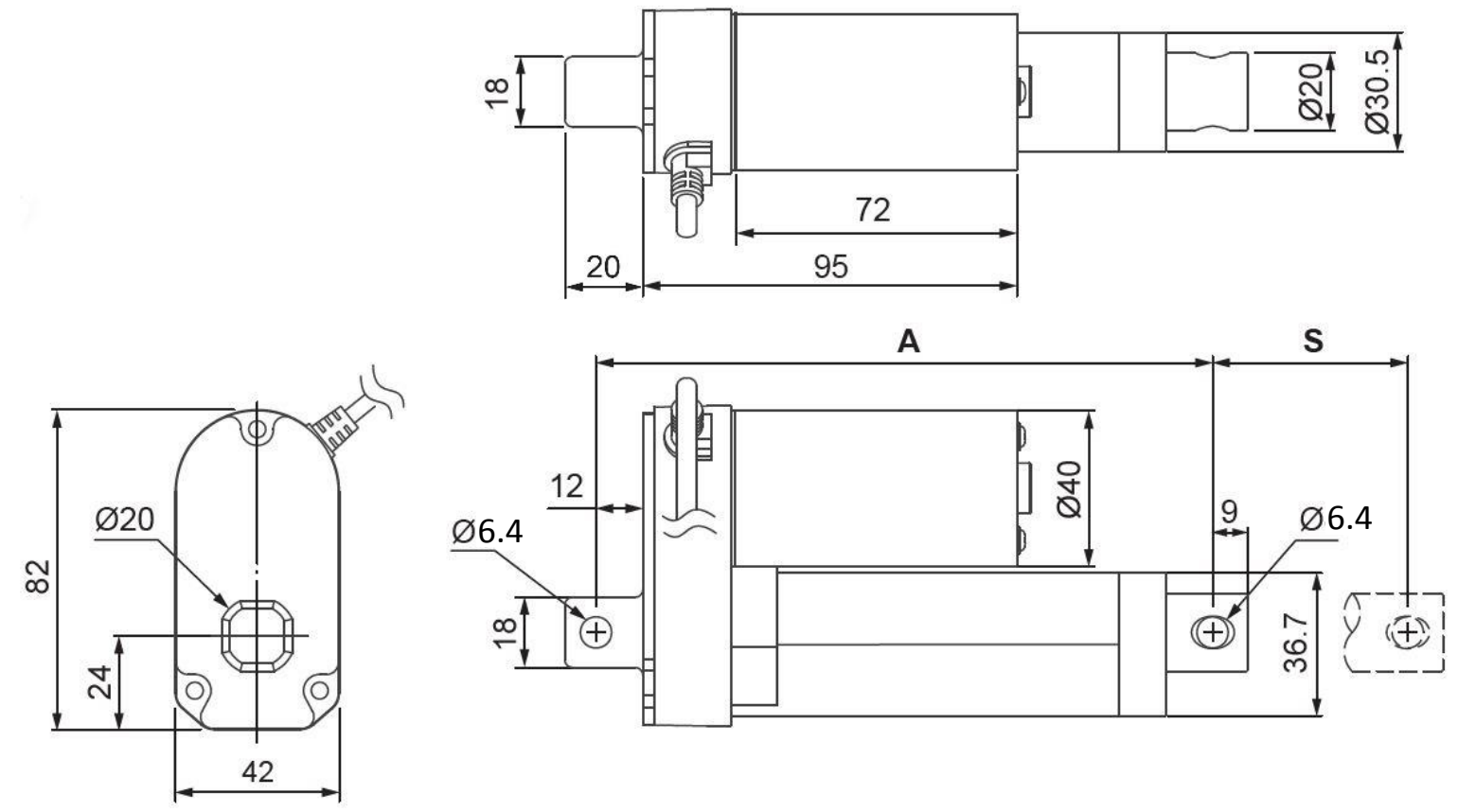

\begin{tabular}{|c|c|c|c|c|c|c|c|c|c|c|c|c|}
\hline $\begin{array}{c}\text { Nominal } \\
\text { Stroke }\end{array}$ & \multicolumn{2}{|c|}{$2 ”$} & \multicolumn{2}{c|}{$4 "$} & \multicolumn{2}{c|}{$6 ”$} & \multicolumn{2}{c|}{$8 ”$} & \multicolumn{2}{c|}{$10 ”$} & \multicolumn{2}{c|}{$12 ”$} \\
\hline & in & $\mathrm{mm}$ & in & $\mathrm{mm}$ & in & $\mathrm{mm}$ & in & $\mathrm{mm}$ & in & $\mathrm{mm}$ & in & $\mathrm{mm}$ \\
\hline $\begin{array}{c}\text { Actual } \\
\text { Stroke } \\
\text { (S) }\end{array}$ & 1.97 & 50 & 3.94 & 100 & 5.91 & 150 & 7.87 & 200 & 9.84 & 250 & 11.81 & 300 \\
\hline $\begin{array}{c}\text { Retracted } \\
\text { Length } \\
\text { (A) }\end{array}$ & 6.22 & 158 & 8.23 & 209 & 10.24 & 260 & 12.24 & 311 & 14.25 & 362 & 16.26 & 413 \\
\hline $\begin{array}{c}\text { Extended } \\
\text { Length }\end{array}$ & 8.19 & 208 & 12.17 & 309 & 16.14 & 410 & 20.19 & 511 & 24.09 & 612 & 28.07 & 713 \\
\hline
\end{tabular}




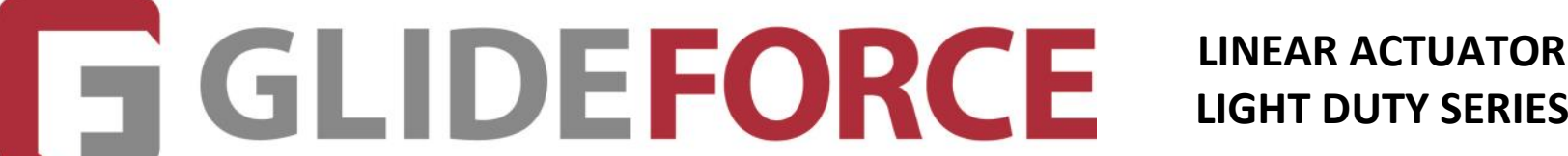

Dimensions - Actuator With Potentiometer
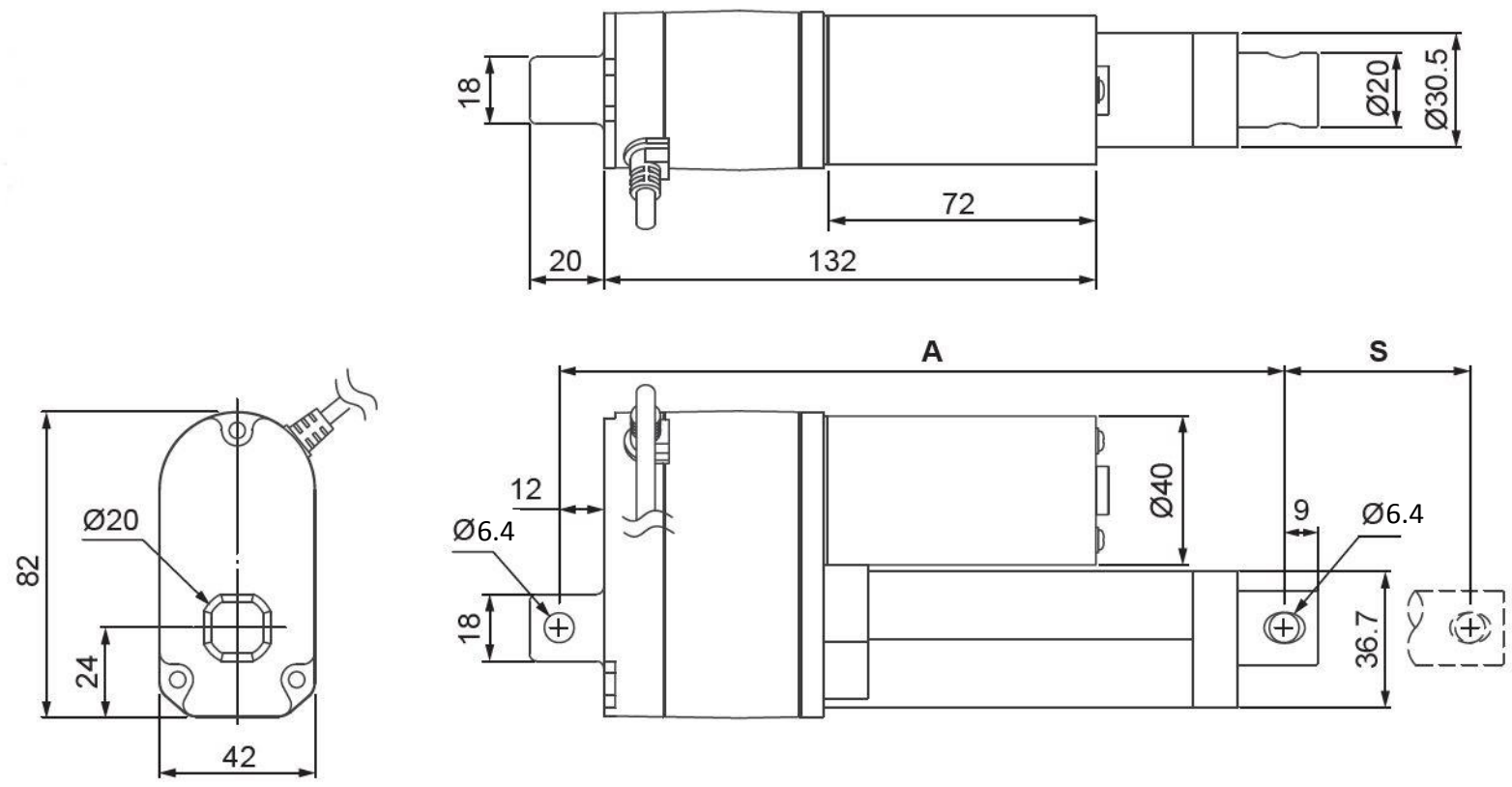

\begin{tabular}{|c|c|c|c|c|c|c|c|c|c|c|c|c|}
\hline $\begin{array}{c}\text { Nominal } \\
\text { Stroke }\end{array}$ & \multicolumn{2}{|c|}{$2 "$} & \multicolumn{2}{c|}{$4 "$} & \multicolumn{2}{c|}{$6 "$} & \multicolumn{2}{c|}{$8 ”$} & \multicolumn{2}{c|}{$10 "$} & \multicolumn{2}{c|}{$12 ”$} \\
\hline & in & $\mathrm{mm}$ & in & $\mathrm{mm}$ & in & $\mathrm{mm}$ & in & $\mathrm{mm}$ & in & $\mathrm{mm}$ & in & $\mathrm{mm}$ \\
\hline $\begin{array}{c}\text { Actual } \\
\begin{array}{c}\text { Stroke } \\
\text { (S) }\end{array}\end{array}$ & 1.97 & 50 & 3.94 & 100 & 5.91 & 150 & 7.87 & 200 & 9.84 & 250 & 11.81 & 300 \\
\hline $\begin{array}{c}\text { Retracted } \\
\text { Length } \\
(\mathrm{A})\end{array}$ & 7.68 & 195 & 9.69 & 246 & 11.69 & 297 & 13.70 & 348 & 15.71 & 399 & 17.72 & 450 \\
\hline $\begin{array}{c}\text { Extended } \\
\text { Length }\end{array}$ & 9.65 & 245 & 13.62 & 346 & 17.60 & 447 & 21.57 & 548 & 25.55 & 649 & 29.53 & 750 \\
\hline
\end{tabular}




\section{5 \\ G LIDEFORCE \\ LINEAR ACTUATOR LIGHT DUTY SERIES}

\section{Performance Data}

\begin{tabular}{|c|c|c|c|c|c|c|}
\hline $\begin{array}{c}\text { Gear } \\
\text { Ratio }\end{array}$ & \multicolumn{2}{|c|}{ Max Load/Dynamic } & \multicolumn{2}{c|}{ Speed/No Load } & \multicolumn{2}{c|}{ Speed/Max Load } \\
\hline & Lbs. [US] & Newtons & inches/sec & $\mathrm{mm} / \mathrm{sec}$ & inches/sec & $\mathrm{mm} / \mathrm{sec}$ \\
\hline $5: 1$ & 34 & 150 & 1.73 & 43.9 & 1.44 & 36.5 \\
\hline $10: 1$ & 56 & 250 & 1.09 & 27.6 & 0.93 & 23.5 \\
\hline $20: 1$ & 112 & 500 & 0.57 & 14.6 & 0.48 & 12.3 \\
\hline $30: 1$ & 180 & 800 & 0.37 & 9.5 & 0.30 & 7.5 \\
\hline $40: 1$ & 225 & 1000 & 0.28 & 7.0 & 0.22 & 5.5 \\
\hline
\end{tabular}

\begin{tabular}{|c|c|c|c|c|}
\hline \multirow{2}{*}{$\begin{array}{c}\text { Gear } \\
\text { Ratio }\end{array}$} & \multicolumn{4}{|c|}{ Typical Current (A) } \\
\cline { 2 - 5 } & \multicolumn{2}{|c|}{ No Load } & Full Load \\
\cline { 2 - 5 } & 12 VDC & 24 VDC & 3.2 & 24 VDC \\
\hline $5: 1$ & 1.2 & 0.6 & 3.2 & 1.6 \\
\hline $10: 1$ & 1.2 & 0.6 & 3.2 & 1.6 \\
\hline $20: 1$ & 1.2 & 0.6 & 3.2 & 1.6 \\
\hline $30: 1$ & 1.2 & 0.6 & 3.2 & 1.6 \\
\hline $40: 1$ & 1.2 & 0.6 & & \\
\hline
\end{tabular}

Speed vs Load

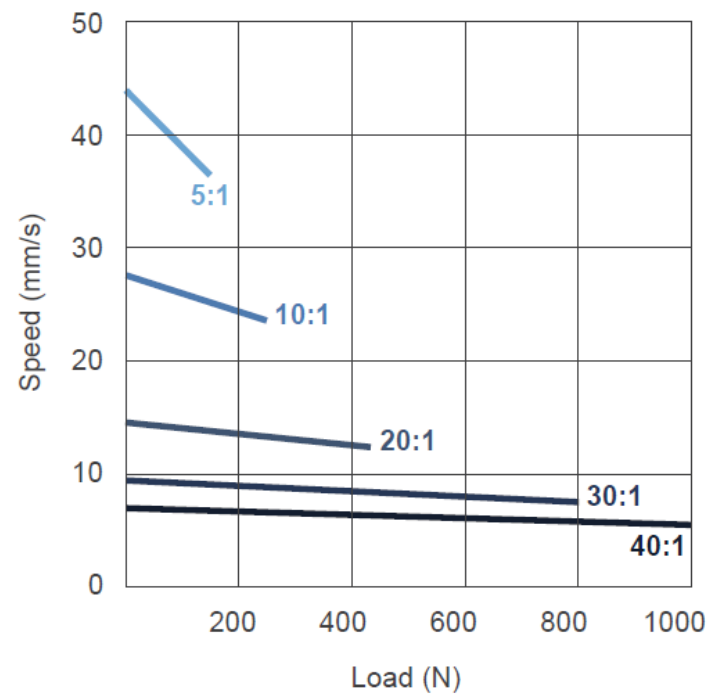

\section{V DC Current vs Load $\quad 12$ V DC}

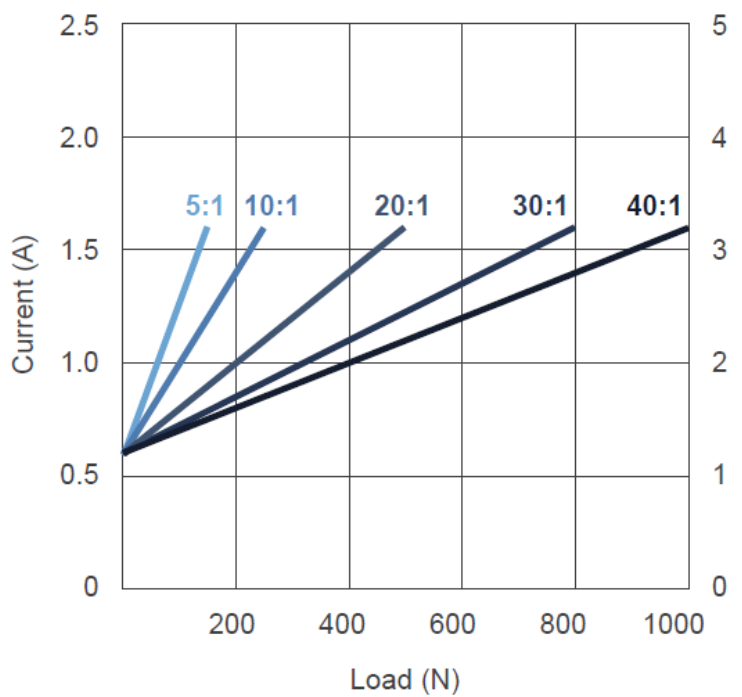

Note: Above graphs and performance data do not apply to the "Q" (quiet) option.

Concentric International 1901 Bell Ave., Suite 18, Des Moines, IA 50315 www.concentricintl.com Rev. 20171208 


\section{F

Electrical

Med Motor Power DC 12V /24V

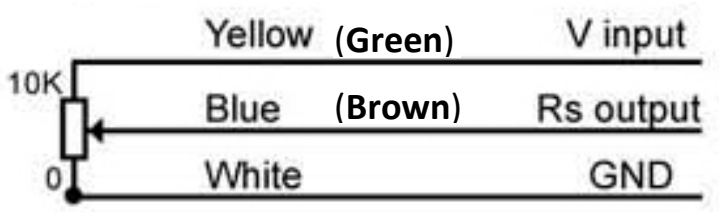

\section{Connector Wiring}

$$
\begin{aligned}
& \text { AMP } \\
& \text { 2-PIN CDNNECTOR } \\
& 106527-2 \\
& \text { AMP TERMINAL } \\
& \text { 106529-2 } \\
& \text { (2 PLACES) }
\end{aligned}
$$

Without Potentiometer

Red + // Black - : Extend

Red - // Black + : Retract

With Potentiometer

Red + // Black - : Retract

Red - // Black + : Extend

\begin{tabular}{|c|c|}
\hline \multicolumn{2}{|c|}{ Value of POT } \\
\hline Stroke (in / mm) & Resistance \\
\hline $1.97 / 50$ & $0.3-9.3 \mathrm{~K}$ \\
\hline $3.94 / 100$ & $0.3-9.7 \mathrm{~K}$ \\
\hline $5.91 / 150$ & $0.3-8.6 \mathrm{~K}$ \\
\hline $7.87 / 200$ & $0.3-9.6 \mathrm{~K}$ \\
\hline $9.84 / 250$ & $0.3-9.3 \mathrm{~K}$ \\
\hline $11.81 / 300$ & $0.3-9.3 \mathrm{~K}$ \\
\hline \multicolumn{2}{|c|}{ Tolerance: +/- 0.3K } \\
\hline $\begin{array}{l}\text { Values in this table are an approximation. } \\
\text { Actual values will vary due to actuator } \\
\text { configuration and construction. }\end{array}$ \\
\hline
\end{tabular}

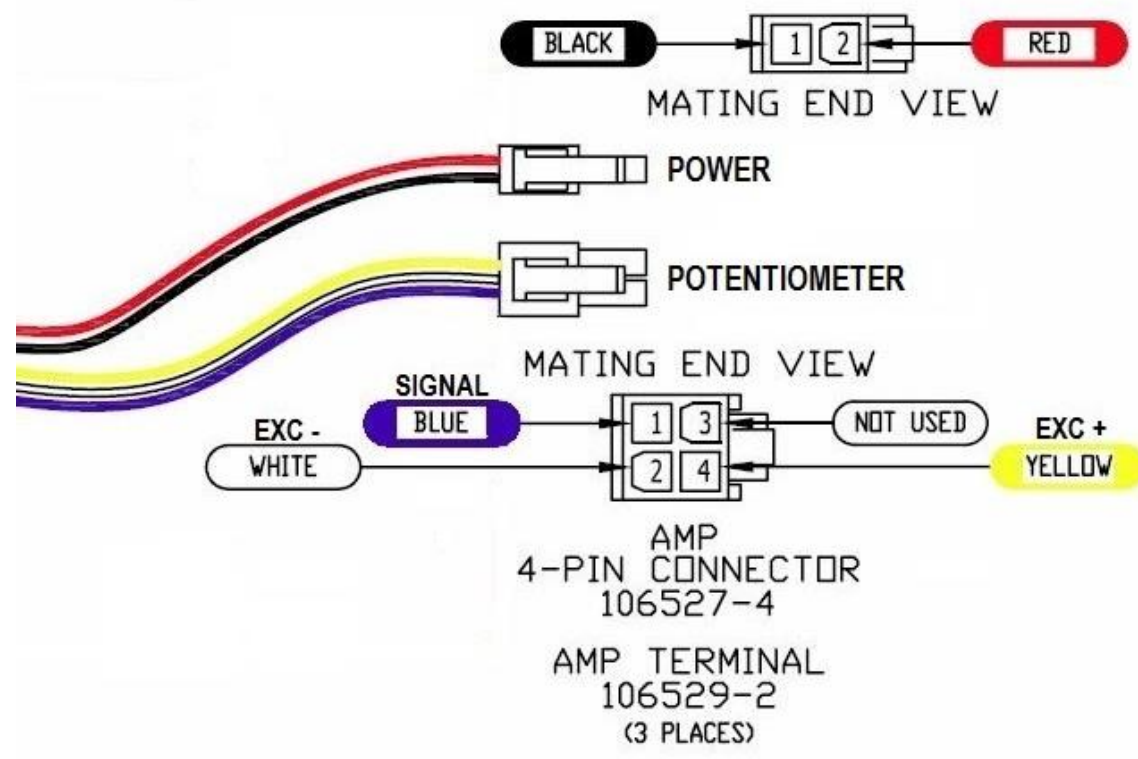




\section{Ordering Key}

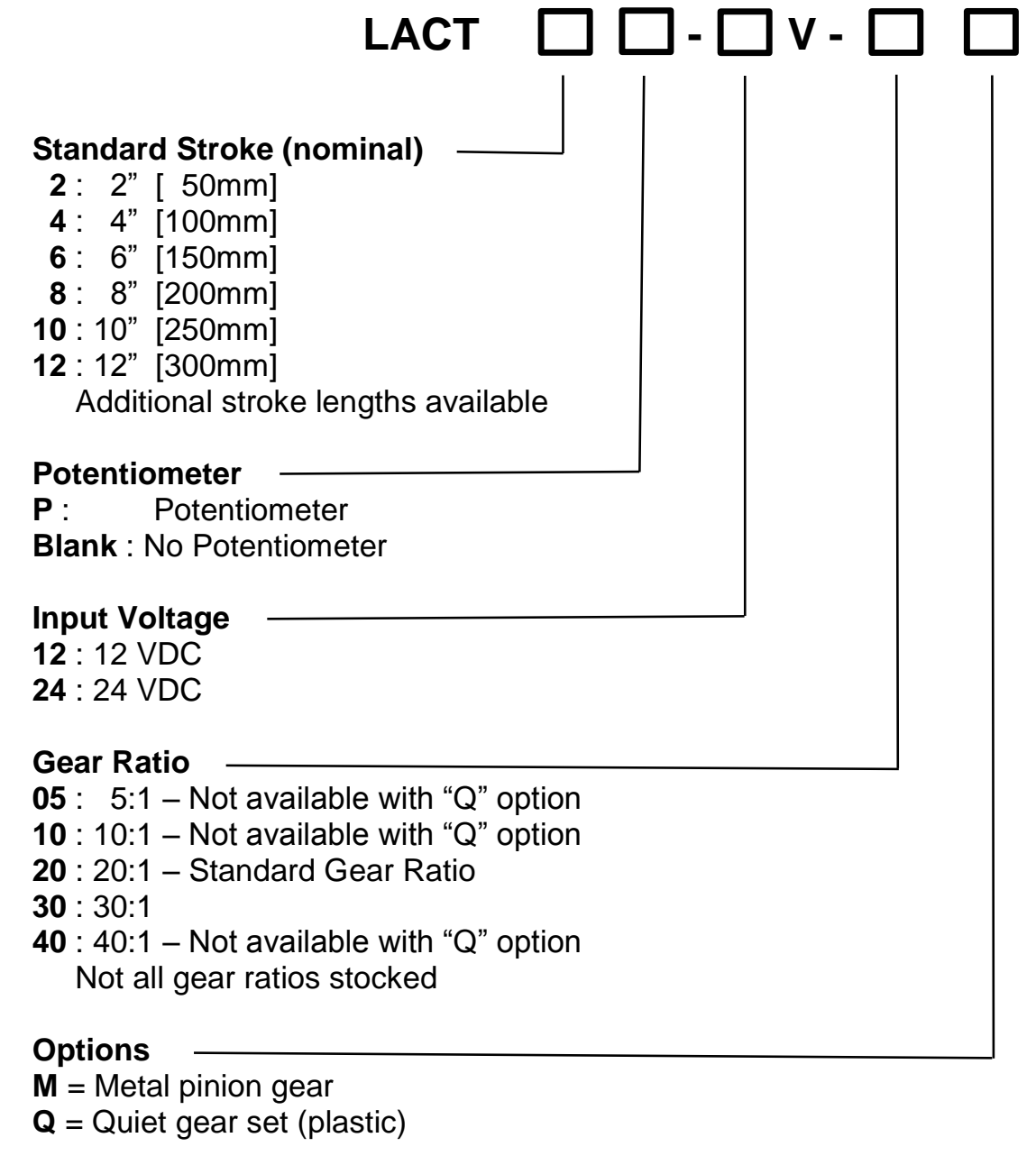

\section{Terms of Use}

The user is responsible for determining the suitability of Concentric International products for specific applications. Due to continuous development in order to improve its products, Concentric International products are subject to change without prior notice. Concentric International reserves the right to discontinue the sale of any products at any time. 